CELSO DE CARVALHO NORONHA NETO

\title{
Matriz de Massa de Ordem Elevada, Dispersão de Velocidades e Reflexões Espúrias
}

Tese apresentada à Escola de Engenharia de São Carlos para obtenção do título de Doutor em engenharia.

Área de concentração: Dinâmica Estrutural.

Orientador: Prof. Dr. José Elias Laier

São Carlos

2008 
AUTORIZO A REPRODUÇÃO E DIVULGAÇÃO TOTAL OU PARCIAL DESTE TRABALHO, POR QUALQUER MEIO CONVENCIONAL OU ELETRÔNICO, PARA FINS DE ESTUDO E PESQUISA, DESDE QUE CITADA A FONTE.

Ficha catalográfica preparada pela Seção de Tratamento da Informação do Serviço de Biblioteca - EESC/USP

Noronha Neto, Celso de Carvalho
Matriz de massa de ordem elevada, dispersão de
velocidades e reflexões espúrias / Celso de Carvalho
Noronha Neto i orientador José Elias Laier. -- São
Carlos, 2008.
Tese (Doutorado-Programa de Pós-Graduação e Área de
Concentração em Eengenharia de Estruturas) -- Escola de
Engenharia de São Carlos da Universidade de São Paulo,
2008.
1. Dinâmica estrutural. 2. Elementos finitos. 3. Ondas
evanescentes. 4. Ondas espúrias. 5. Precisão numérica.
Timoshenko. I. Título.


FOLHA DE JULGAMENTO

Candidato: Engenheiro CELSO DE CARVALHO NORNHA NETO

Tese defendida e julgada em 16/05/2008 perante a Comissão Julgadora:

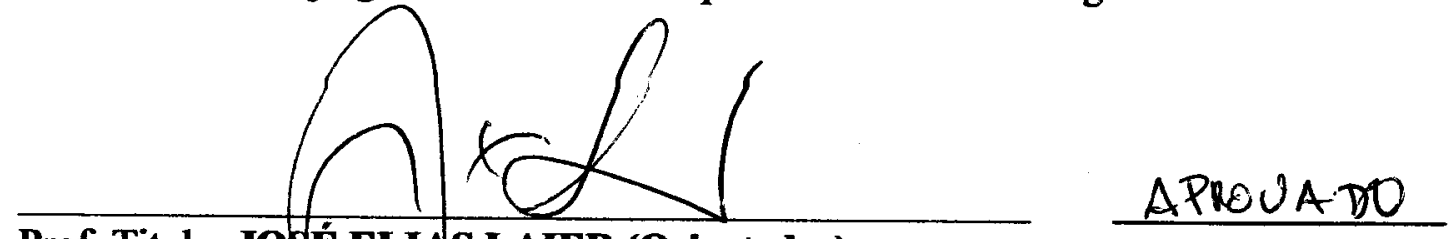

Prof, Titular JOAÉ ELIAS LAIER (Orientador)

(Escola de Engenparia de São Carlos/USP)

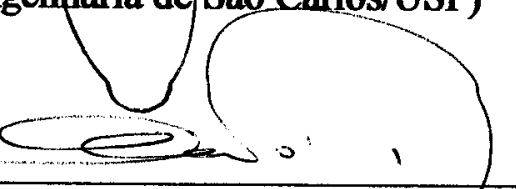

Aprovado

Prof. Dr. MARIO FRANCISCO MUCHERONI

(Escola de Engenharia de São Carlos/USP)
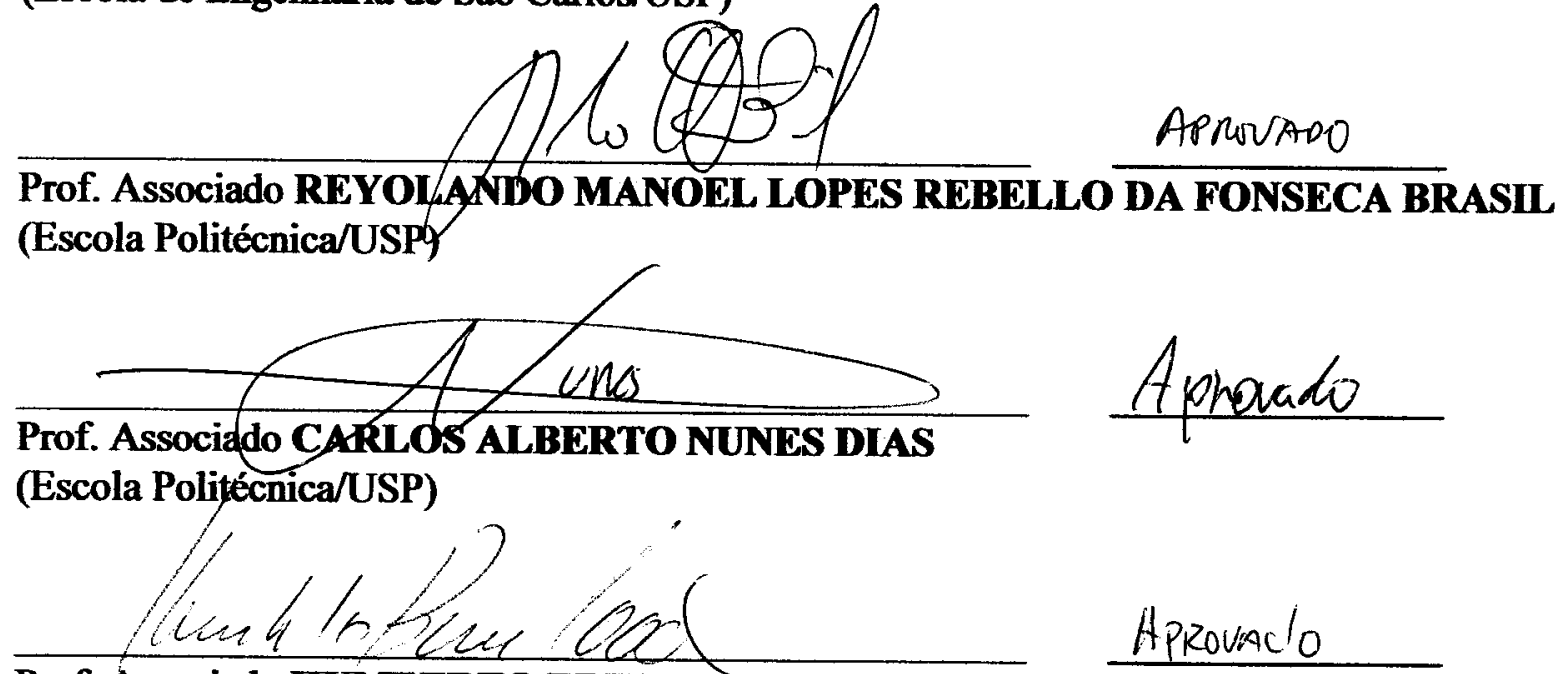

Prof. Associado HUMBERTO BREVES CODA

(Escola de Engenharia de São Carlos/USP)

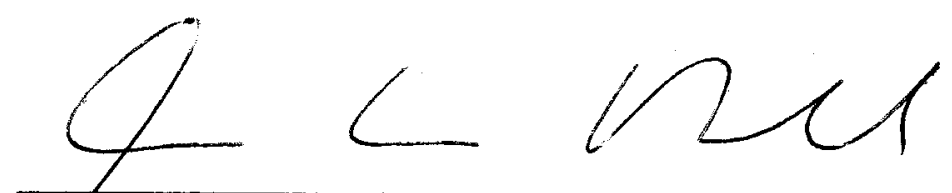

P.of. Associado MARCIO ANTONIO RAMALHO

Coordenador do Programa de Pós-Graduação em

Engenharia Civil (Engenharia de Estruturas)

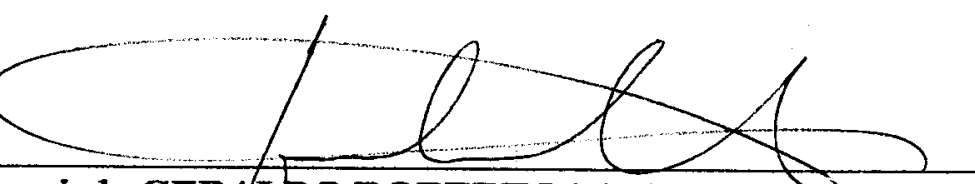

Prof. Associado GERAIDO ROBERTO MARTINS DA COSTA

Presidentéa Comissão de Pós-Graduação 
AGRADECIMENTOS

À EESC-USP pelo suporte físico e à Capes pelo suporte financeiro. 
ÍNDICE

Simbologias__ 01

Termos técnicos__ 03

CAPÍTULO 1 -

Introdução 04

CAPÍTULO 2 -

Propagação Ondulatória e Método dos Elementos Finitos:

Elementos de Barra 07

2.1. Introdução 07

2.2. Equação de movimento 08

2.3. Estudo da dispersão numérica 14

2.4. Exemplo de aplicação 19

2.5. Reflexões espúrias 23

CAPÍTULO 3 -

Algoritmos de Integração para Elementos de Barra com Quadraturas de Ordem mais Elevada e Matriz de Massa 30

3.1. Introdução 30

3.2. Combinação otimizada 31

3.3 Integrador espacial proposto 35

3.4. Resultados 39

CAPÍTULO 4 -

Propagação numérica em elementos simples de viga de Timoshenko 44

4.1. Introdução 44 
4.2. Equação de movimento 45

4.3. Cálculo da propagação numérica 49

4.4 Cálculo da reflexão espúria 59

CAPÍTULO 5 -

Algoritmos de Integração para Elementos de Viga 64

5.1. Introdução 64

5.2. Formulação mediante integrador espacial do tipo $\beta_{\mathrm{S}}$ 65

5.3. Formulação generalizada 68

5.4. Resultados numéricos 73

CAPÍTULO 6 -

Considerações Teóricas Complementares para a Elaboração de Algoritmos de Integração para Elementos Finitos em Análises Dinâmicas 82

6.1. Introdução 82

6.2. Elevação da ordem de erro dos resíduos em elementos de viga 83

6.3. Equilíbrio de forças transversais em elementos de viga 87 CAPÍTULO 7 -

Conclusões 94

Referências 97 


\section{LISTA DE SÍMBOLOS}

\section{Geral:}

- Cada ponto sobrescrito a uma variável indica uma derivação em relação ao tempo;

- Cada apóstrofe acompanhando uma variável indica uma derivação em relação ao espaço;

- Valores numéricos entre parênteses indicam expressões e igualdades matemáticas;

- Valores numéricos entre colchetes indicam referências bibliográficas;

- Letras entre colchetes indicam matrizes;

- Letras entre chaves indicam vetores;

- A letra T (maiúscula) sobrescrita à direita de um par de chaves ou de colchetes indica a transposta do vetor ou da matriz respectivamente;

- A abreviação SIM no canto inferior esquerdo de uma matriz indica simetria;

\section{Descrição dos parâmetros:}

N__ Número de onda;
Número de onda relativo ao problema discreto;
Variável auxiliar de alocação dinâmica;


Amplitude da onda incidente;

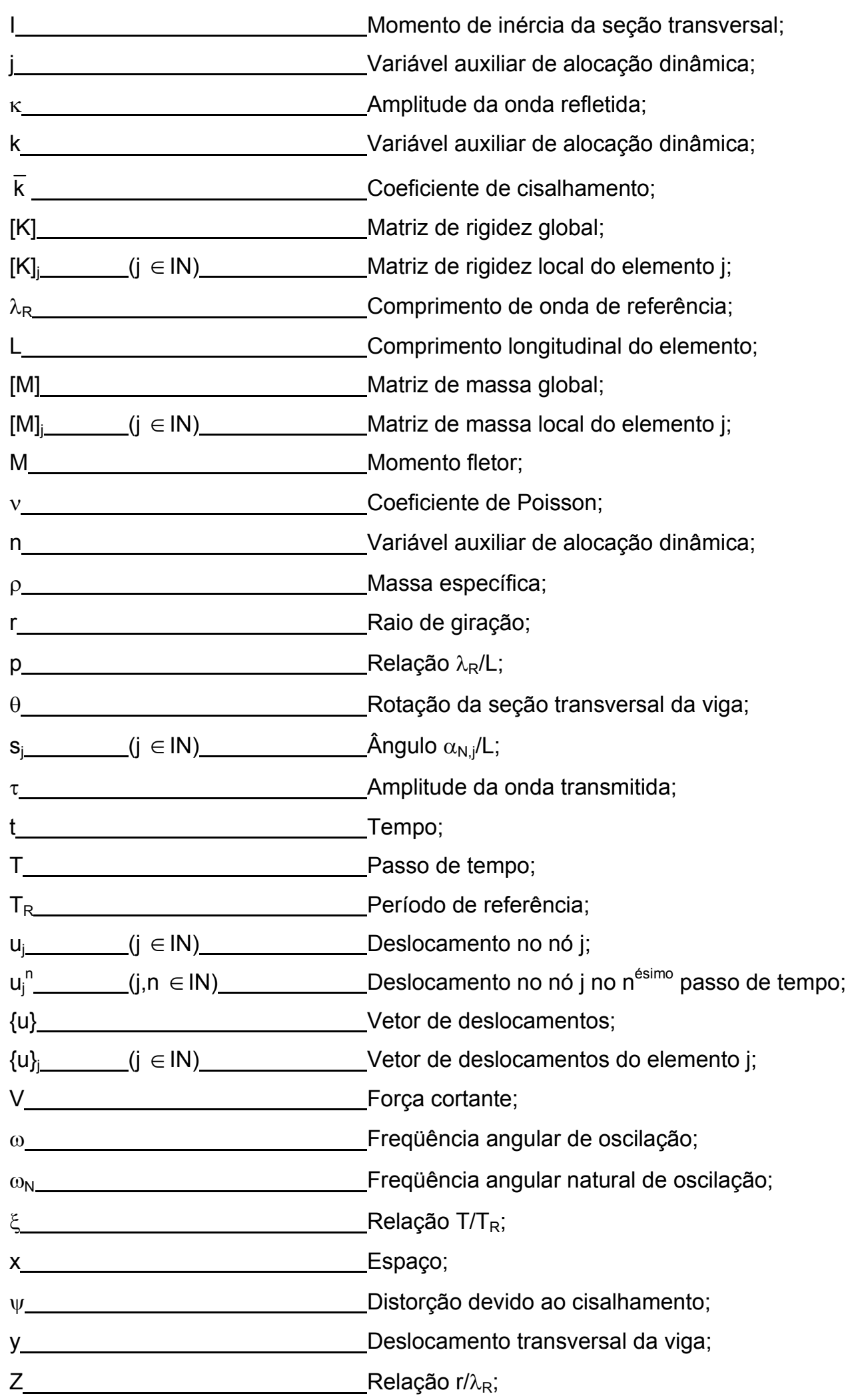




\section{TERMOS TÉCNICOS}

\begin{tabular}{|c|c|}
\hline ispersão_ & Erro associado à imprecisão da integração numérica; \\
\hline Dispersão de velocidade & Erro do valor numérico da velocidade de propagação; \\
\hline Efeito espúrio_ & $\begin{array}{l}\text { Fenômeno que ocorre no problema numérico, mas não no } \\
\text { teórico; }\end{array}$ \\
\hline Equação de diferenças_ & $\begin{array}{l}\text { Aquela que resume a equação regente em termos } \\
\text { discretos no tempo e no espaço; }\end{array}$ \\
\hline Erro de truncamento & $\begin{array}{l}\text { Erro proveniente da expansão em série dos termos } \\
\text { envolvidos na equação de diferenças; }\end{array}$ \\
\hline Freqüência espacial__ & O inverso do comprimento de onda; \\
\hline Integração numérica_ & $\begin{array}{l}\text { Descrever um parâmetro ou uma equação diferencial } \\
\text { através de um sistema discreto; }\end{array}$ \\
\hline Matrix de massa consistente_ & _Modelo clássico com matriz de massa "cheia"; \\
\hline Matriz de massa concentrada & $\begin{array}{l}\text { Aquela que possui apenas os termos da diagonal principal } \\
\text { como não nulos; }\end{array}$ \\
\hline Onda evanescente_ & $\begin{array}{l}\text {-Vibração dotada de um decaimento exponencial, em } \\
\text { amplitude, ao longo do sentido de propagação (domínio do } \\
\text { espaço); }\end{array}$ \\
\hline Ordem de erro_ & $\begin{array}{l}\text { Potência relativa à primeira parcela restante da expansão } \\
\text { em séries dos termos discretos envolvidos; }\end{array}$ \\
\hline Ponto de bifurcação_ & _Valor onde a raíz de uma equação assume valor complexo; \\
\hline Quadratura numérica & _Sinônimo de integração numérica; \\
\hline Reflexão espúria_ & $\begin{array}{l}\text { Onda refletida na interface de dois elementos adjacentes, } \\
\text { devido à diferença geometrica entre eles; }\end{array}$ \\
\hline
\end{tabular}




\section{RESUMO}

NORONHA NETO, CELSO C. Matriz de Massa de Ordem Elevada, Dispersão de Velocidades e Reflexões Espúrias. 1995. 100 f. Tese (Doutorado) - Escola de Engenharia de São Carlos, Universidade de São Paulo, São Carlos, 2008.

O assunto principal deste trabalho é qualificar, quantificar e implementar o comportamento numérico de estruturas discretizadas através do método dos elementos finitos. Serão abordados apenas os elementos lineares unidimensionais dinâmicos, porém a aplicabilidade da formulação proposta pode se estender para elementos bi e tridimensionais lineares dinâmicos. Inicia-se com uma introdução ao tema. Com certo desenvolvimento matemático, pode-se isolar analiticamente a parcela relacionada ao erro numérico. Elevando a ordem do erro de truncamento, obtém-se precisão elevada na resposta numérica. Inspirado no integrador temporal de Newmark, projetam-se elementos que apresentam estabilidade incondicional para os chamados efeitos espúrios. O efeito evanescente é um fenômeno espúrio onde a onda se propaga ao longo da estrutura acompanhada de um amortecimento puramente numérico ao longo do domínio do espaço. Outro efeito analisado é a reflexão espúria. Quando dois elementos adjacentes têm comprimentos diferentes, surge uma onda de reflexão (ou duas, no caso do elemento de viga) na interface deles. Tal onda, também de origem puramente matemática, existe devido à diferença entre as massas e as rigidezes absolutas dos elementos envolvidos, independente do fato de que eles tenham as mesmas características físicas. A relação entre o incremento de tempo e o período de oscilação é convenientemente empregada como principal parâmetro para quantificar a discretização no domínio temporal. No domínio do espaço, a relação empregada é entre o comprimento do elemento e o comprimprimento de onda.

Palavras-chave: Dinâmica estrutural, Elementos finitos, Ondas evanescentes, Ondas espúrias, Precisão numérica, Timoshenko. 


\section{ABSTRACT}

\section{NORONHA NETO, CELSO C. High Order Mass Matrix, Velocity}

Dispersion and Spurious Wave Reflection. 100 p. Thesis (Doctor Degree) - Engineer School of São Carlos, University of São Paulo, São Carlos, 2008.

The main subject of this work is to qualify, quantify and implement the numerical behavior of discrete structures through the finite element method. It will be investigated only the dynamic onedimensional linear elements, but the applicability of the proposed formulation can be extended to the bi and tri-dimensional cases. It begins with an introduction to the theme. With some mathematical development, the related numerical error can be isolated analytically. Once the truncation error is isolate, a high precision numerical response is obtained. Inspired in the Newmark time integrator, unconditionally stable elements for spurious effects are idealized. The evanescent effect is a spurious phenomenon where the wave propagates along the structure subjected to a numerical damping in the spatial domain. Another effect analyzed here is the spurious wave reflection. When two adjacent elements have different lengths, a reflected wave exists (two waves for the beam element) at their interface. This wave, which meaning is purely mathematical, exists due to the difference of their absolute mass and stiffness between the finite elements involved, even when both elements have the same physical properties. The rate between the time increment and the period of oscillation is conveniently employed as the main parameter to quantify the time discretization. In the spatial domain, the used parameter is the relation between the element and the wave length.

Keywords: Structural dynamics, Finite element, Evanescent waves, Spurious wave reflections, Numerical precision, Timoshenko. 


\section{Capítulo 1}

\section{Introdução}

O constante interesse por parte do setor industrial, impulsionado pelo mercado já em nível globalizado, de desenvolver produtos cada vez mais competitivos é responsável pela exponencial taxa com que surgem novos recursos computacionais direcionados ao ramo da engenharia, onde, inevitavelmente, o comportamento estrutural do produto fabricado deve ser investigado para diversas condições. Entretanto, a limitação constante de uma análise estrutural por elementos finitos se encontra na velocidade de resolução algébrica, na precisão das respostas obtidas e na estabilidade do sistema. Assim, é comum o uso de computadores especiais dotados de diversos processadores para análises estruturais que, ainda assim, perduram alguns dias de processamento. 
O estudo apresentado é direcionado para análises lineares dinâmicas, envolvendo propagação ondulatória, análise transiente e decomposição modal. Embora a não-linearidade esteja presente na maioria dos códigos computacionais encontrados, tais análises estruturais dinâmicas são intrinsicamente lineares.

A formulação de elementos finitos com elevada precisão numérica almeja contribuir na redução do tempo de processamento, uma vez que possibilita editar malhas menos refinadas. Conjuntamente, também se tem alívio computacional quando se trabalha com matrizes de massa concentrada.

Certos tipos de sistemas estruturais, assim como de componentes estruturais isolados, têm melhor representatividade e performance quando modelados por elementos de geometria unidimensional, tal qual o de barra (treliça) e o de viga (pórtico). A indústria automobilística, por exemplo, emprega elementos de viga no modelamento do chassis, travessas colunas no pré-projeto de um veículo, além de outros componentes como o sistema de suspensão, acoplamento de malhas, pontos de solda, etc. Muitos componentes estruturais na indústria de óleo e gás são dutos (Risers, tubos enterrados, gasodutos, etc.). Como exemplos de aplicação na indústria aeroespacial, têm-se os stringers (barras de reforço de painéis) e as vigas da fuselagem. Em geral, o tempo de processamento e a estabilidade do sistema numérico são fatores críticos em um projeto. Tem-se ainda que, em um processo de otimização física ou topológica (D.O.E.), onde um modelo estrutural discretizado é processado várias vezes seguidas, a redução do tempo consumido pela resolução do sistema matricial significa uma economia considerável no duração total de execução.

Neste trabalho, propõe-se qualificar, quantificar e elaborar uma formulação de elementos finitos com melhor desempenho em relação à freqüência de oscilação no domínio do tempo e do espaço. Como sabido, a propagação ondulatória em um modelo de elementos finitos tem natureza dispersiva, onde o efeito da imprecisão numérica ocasiona alongamento ou encurtamento do período de oscilação e do comprimento de onda. Isto afeta, por conseqüência, a velocidade de propagação das ondas. Além disso, algumas singularidades numéricas podem ocasionar a falha de toda a análise estrutural. Uma introdução a 
este tema é feita no capítulo 2, onde elege-se abordar o elemento finito consistente clássico de barra, com uma grau de liberdade em cada extremidade, por se tratar do mais simplificado dos casos.

Ainda no capítulo 2, insere-se o leitor no cálculo da quantificação de dois efeitos espúrios, ou seja, que ocorrem no problema numérico mas não no teórico. Tais efeitos interferem substancialmente na validação de um cálculo estrutural. Como primeiro fenômeno a ser apontado tem-se a chamada onda evanescente, que é caracterizada por um decaimento exponencial da amplitude da onda ao longo de seu eixo de propagação. Em seguida, discute-se o caso da reflexão espúria, intrinsicamente presente em malhas com elementos finitos de diferentes dimensões, dita malha não homogênea. Tal onda surge para garantir o equilíbrio de um nó compartilhado por dois elementos de comprimento diferentes. Assim, por uma questão de compatibilidade física, uma onda incidente reflete-se na interface inter-elementar.

O entendimento do comportamento numérico do clássico elemento finito de barra permite a elaboração de integradores visando o suprimento dos critérios de precisão e de estabilidade perante os efeitos espúrios. O capítulo 3 avança em direção a este objetivo, resultando em um tipo de formulação otimizada que é idéia central do estudo proposto.

Uma vez compreendido o que ocorre numericamente com o elemento finito simples de barra, parte-se para o capítulo 4, que aplica o mesmo estudo ao elemento finito unidimensional de viga. Adota-se o modelo constitutivo de acordo com a teoria de viga segundo Timoshenko. No capítulo seguinte, estuda-se o procedimento para criar duas equações de diferenças (compostas por termos discretos) que atendam às equações diferenciais de equilíbrio de força cortante e de momento fletor. A princípio, tal equação de diferenças pode ser entendida como uma combinação de dados numéricos, possibilitando assim a manipulação das variáveis visando obter elevado desempenho para o sistema. Existem algumas analogias interessantes entre o elemento de barra e o de viga, que são apontadas no texto em momentos oportunos. 


\section{CAPÍtulo 2}

\section{Propagação Ondulatória e Método dos Elementos Finitos: Elementos de Barra}

\subsection{INTRODUCCÃO}

Procura-se neste capítulo apresentar o estudo da dispersão da velocidade de propagação ondulatória em decorrência de abordagem numérica das equações de movimento. Trata-se, pois, do estudo de um erro de aproximação do tipo global, cuja natureza é de origem puramente numérica, e que decorre da modelagem do comportamento estrutural pelo emprego de métodos numéricos de integração [MIKLOWITZ (1960), LIU (1994)]. Com esse propósito, as atenções vão estar aqui voltadas para a modelagem empregando-se o mais simples dos elementos finitos, que é o elemento finito de barra com um grau de liberdade por nó de extremidade. 
Uma vez definido um campo de deslocamentos aproximado no domínio do elemento correspondente, a equação diferencial que governa o comportamento, advinda do equilíbrio dinâmico, passa a ser representada por uma equação de diferenças, a qual exprime numericamente o comportamento do sistema agora discretizado. Dessa forma, o equilíbrio de ações passa a ser expresso segundo um sistema de equações algébricas, explicitando-se, pois, segundo notação matricial clássica. No caso do elemento simples de barra, a equação de movimento em sua versão numérica se reduz a apenas uma única equação algébrica.

A propagação ondulatória em sua versão numérica correspondente, bem como a presença das chamadas ondas evanescentes, são abordados analítica e numericamente, chamando-se a atenção para a correspondência entre tais formulações matemáticas, assim como a correspondência entre os resultados da via analítica e os da via numérica (sistemas discretizados).

Entende-se por onda evanescente aquela solução da equação de movimento que não apresenta característica de propagação, e que está sujeita a um amortecimento segundo a variável espacial, pela presença de um fator exponencial com potência negativa envolvendo a variável espaço (dessa característica decorre a denominação evanescente). Conforme será oportunamente mostrado, outro fenômeno de natureza também puramente numérica são as chamadas reflexões espúrias. Este último fenômeno, também proveniente da integração numérica da equação de movimento, ocorre na interseção de elementos finitos de dimensões diferentes, ou seja, quando do emprego de malhas de elementos finitos geometricamente não uniformes.

\subsection{EQUAÇÃO DE MOVIMENTO}

A abordagem analítica clássica da equação ondulatória (onda de D’Alembert) decorre do equilíbrio axial de barra, e assim se expressa:

$$
c^{2} \frac{\partial^{2} u}{\partial x^{2}}-\frac{\partial^{2} u}{\partial t^{2}}=0
$$


onde c é a velocidade de propagação dada por:

$$
c=\sqrt{\frac{E}{\rho}}
$$

sendo $\mathrm{E}$ o módulo de elasticidade do material da barra e $\rho$ sua massa específica. Como se vê de (2.1), a equação diferencial em questão não privilegia, em termos do grau de derivação, nenhuma das variáveis (espaço e tempo). Em outras palavras, a história no tempo do movimento é, a menos da constante c (velocidade de propagação), a mesma que pode ser observada no espaço.

A solução geral de (2.1) tem a seguinte redação em notação complexa:

$$
u(x, t)=C_{1} e^{\frac{2 \pi \mathrm{i}}{\lambda}(x-c t)}+C_{2} e^{\frac{2 \pi \mathrm{i}}{\lambda}(x+c t)}
$$

onde $\lambda$ é o comprimento da onda, c a velocidade de propagação da onda (dada pela relação entre o comprimento de onda e o período de oscilação), "i" a unidade imaginária, "e" a base do logarítimo natural e $C_{1}$ e $C_{2}$ são constantes de integração dependendes das condições de contorno. A primeira parcela do membro direito de (2.3) corresponde a uma onda propagando no sentido positivo do eixo coordenado Ox, e o segundo a uma onda propagando-se no sentido negativo.

$\mathrm{Na}$ dinâmica de estruturas, a formulação do método dos elementos finitos tem como principal objetivo a integração das equações diferenciais do movimento através de uma discretização espacial do problema (integração por subdomínios), seguida da integração temporal (integração passo-a-passo).

Como sabido, para cada elemento k têm-se correspondentemente uma matriz de massa, de amortecimento e de rigidez, referidas como $[\mathrm{M}]_{\mathrm{k}},[\mathrm{C}]_{\mathrm{k}}$ e $[\mathrm{K}]_{\mathrm{k}}$ respectivamente. O vetor que contempla os deslocamentos nos nós do elemento é chamado de vetor de deslocamentos, cuja simbologia é representada por $\{u\}_{k}$. Operando-se sobre as matrizes individuais dos elementos finitos mediante 0 auxílio de uma matriz de alocação, que relaciona os graus de liberdade locais do elemento (coordenadas locais) com os do conjunto (coordenadas globais), obtêmse as matrizes globais de massa [M], de amortecimento [C] e de rigidez $[\mathrm{K}] \mathrm{da}$ 
estrutura. Desta maneira, o método dos elementos finitos permite explicitar o equilíbrio dinâmico da estrutura segundo um sistema de equações diferenciais na forma:

$$
[\mathrm{M}]\{\ddot{\mathrm{u}}\}+[\mathrm{C}]\{\dot{\mathrm{u}}\}+[\mathrm{K}]\{\mathrm{u}\}=\{\mathrm{f}\}
$$

onde $\{f\}$ representa o vetor de ações externas, e a notação com pontos superiores indica o grau de derivação em relação ao tempo. Neste capítulo, a atenção vai estar voltada apenas para sistemas dinâmicos não amortecidos fisicamente (propagação ondulatória não amortecida).

O número de equações do sistema em (2.4) é igual ao número de graus de liberdade global da estrutura considerado na modelagem, sendo que cada equação isoladamente vem a ser um equilíbrio de ações segundo uma das coordenadas. Por exemplo, a j $\mathrm{j}^{\mathrm{a}}$ linha do sistema matricial (2.1) refere-se à equação de movimento do joำ grau de liberdade.

Ao se solicitar uma estrutura por meio de uma ação externa ou de um deslocamento imposto, a energia se propaga ao longo da estrutura através de ondas. Todavia, ao se proceder a integração das equações de equilíbrio empregando-se o método dos elementos finitos, além de se ter naturalmente uma resposta aproximada para os deslocamentos; tem-se, como conseqüência, que o comportamento resultante é um movimento ondulatório com velocidade de propagação também aproximada; e de natureza dispersiva, uma vez que a velocidade numérica passa a depender, via de regra, do comprimento de onda.

A figura 2.1 ilustra um elemento finito de barra genérico $\mathrm{k}$ com dois graus de liberdade e de comprimento L. Em cada nó de extremidade (aqui denominados 1 e 2) é considerado haver um grau de liberdade relativo ao movimento axial denominado $u_{j}$, que representa, pois, o movimento axial em cada extremidade.

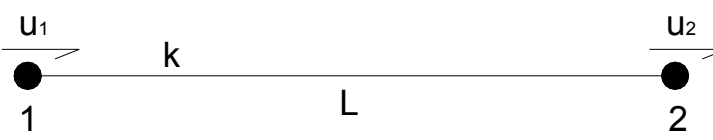

Figura 2.1 - Elemento finito de barra com dois graus de liberdade. 
As matrizes de rigidez e de massa obtidas segundo a modelagem comum consistente (funções de forma lineares) do método dos elementos finitos são, para o elemento em apreço, dadas por:

$$
[\mathrm{K}]_{\mathrm{k}}=\frac{\mathrm{EA}}{\mathrm{L}}\left[\begin{array}{cc}
1 & -1 \\
-1 & 1
\end{array}\right] \quad ; \quad[\mathrm{M}]_{\mathrm{k}}=\frac{\rho \mathrm{AL}}{6}\left[\begin{array}{ll}
2 & 1 \\
1 & 2
\end{array}\right]
$$

onde A é a área da seção transversal da barra.

O procedimento para se efetuar o equilíbrio de forças em um nó j de um elemento é a admissão de uma barra de comprimento infinito sujeita a uma onda incidente que se propaga no seu sentido axial, conforme o ilustrado na figura 2.2. Nesta representação, a nomenclatura empregada para representar o campo de deslocamentos é $u_{j}^{n}$, que indica o deslocamento do nó j no $n \stackrel{\text { ésimo }}{ }$ passo de tempo [WANG (1992)].

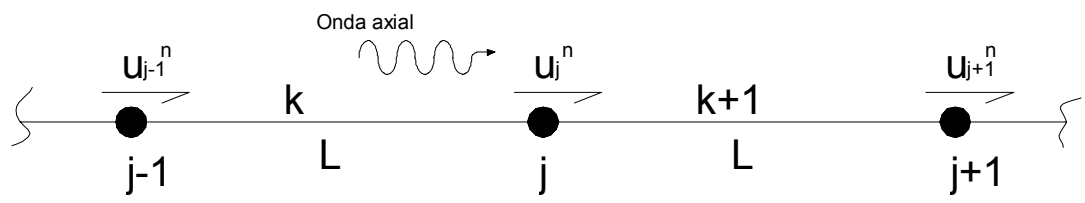

Figura 2.2 - Barra de comprimento infinito sujeita a uma onda uniaxial.

De acordo com o ilustrado na figura 2.2, sendo admitido que não haja força externa aplicada no nó j, o equilíbrio dinâmico referente esse nó, tendo-se em vista (2.5) passa a se expressar [AHMADIAN (1998)]:

$$
\frac{E A}{L}\left(-u_{j-1}^{n}+2 u_{j}^{n}-u_{j+1}^{n}\right)+\frac{\rho A L}{6}\left(\ddot{u}_{j-1}^{n}+4 \ddot{u}_{j}^{n}+\ddot{u}_{j+1}^{n}\right)=0
$$

onde o expoente $\mathrm{n}$ indica que se trata do equilíbrio num passo de tempo genérico, uma vez que a discretização do tempo ainda não foi considerada. A equação (2.5), que é do tipo diferencial (no tempo) de diferença (no espaço), numa redação mais oportuna, se expressa:

$$
\frac{6 E}{\rho L^{2}}\left(-u_{j-1}^{n}+2 u_{j}^{n}-u_{j+1}^{n}\right)+\left(\ddot{u}_{j-1}^{n}+4 \ddot{u}_{j}^{n}+\ddot{u}_{j+1}^{n}\right)=0
$$


sendo este o momento de observar que, numericamente, tal equação ocupa o lugar da equação diferencial (2.1).

Tendo-se em mente que a solução da equação diferencial de diferença (2.7) deve retratar uma propagação ondulatória como (2.1), e que a variável contínua $x$ é agora substituída pela variável inteira j $(x=j L)$, a solução de (2.7) deve ser do tipo [MULLEN (1982)]:

$$
u_{j}^{n}=C \cdot e^{i\left(j \alpha_{N} L-n \omega T\right)}
$$

onde $C, \alpha_{N}$ e $\omega$ são respectivamente a amplitude da onda incidente, freqüência espacial relativa ao problema discreto e freqüência angular de oscilação da onda. Vale assinalar nesse ponto que, embora no tempo o problema ainda não foi discretizado, já adota-se em (2.8) para o tempo uma notação discreta; onde por hora considere-se $t=n T$, sendo $T$ o passo de tempo (incremento no tempo) e $n$ a variável que adiante será discreta. Assim sendo, para os nós j-1 e j+1 têm-se:

$$
\begin{aligned}
& u_{j-1}{ }^{n}=e^{-i \alpha_{N} L} u_{j}{ }^{n} \\
& u_{j+1}{ }^{n}=e^{i \alpha_{N} L} u_{j}{ }^{n}
\end{aligned}
$$

Analogamente, considerando-se os passos de tempo $n-1$ e $n+1$ têm-se como válidas as seguintes redações:

$$
\begin{aligned}
& u_{j}^{n-1}=e^{-i \omega T} u_{j}{ }^{n} \\
& u_{j}^{n+1}=e^{i \omega T} u_{j}{ }^{n}
\end{aligned}
$$

Empregando-se na integração segundo a variável tempo o clássico integrador de Newmark [Newmark (1959)], que pode ser sumarizado em termos dos seguintes operadores hermitianos: 


$$
\begin{aligned}
& \dot{u}_{j}^{n+1}=\dot{u}_{j}^{n}+\left[(1-\delta) \ddot{u}_{j}^{n}+\delta \ddot{u}_{j+1}^{n}\right] T \\
& u_{j}^{n+1}=u_{j}^{n}+\dot{u}_{j}^{n} T+\left[\left(\frac{1}{2}-\beta\right) \ddot{u}_{j}^{n}+\beta \ddot{u}_{j+1}^{n}\right] T
\end{aligned}
$$

onde, como já referido, T é o passo de tempo (incremento), $\delta$ e $\beta$ são parâmetros livres. Tem-se, no caso do operador trapezoidal $(\delta=1 / 2)$, a seguinte relação entre a aceleração e o deslocamento:

$$
\nabla \mathbf{u}_{j}{ }^{n}=\mathbf{T}^{2}(1+\beta \nabla) \dot{\mathbf{u}}_{\mathrm{j}}{ }^{\mathrm{n}}
$$

onde $\nabla$ representa o operador de diferença:

$$
\nabla u_{j}^{n}=u_{j}^{n-1}-2 u_{j}^{n}+u_{j}{ }^{n+1}
$$

Em outras palavras, a eq. (2.12) permite exprimir a aceleração em termos dos deslocamentos. Com isso, tendo-se em vista (2.12), a equação diferencial de diferença (2.7) permite uma nova redação, ou seja:

$$
\nabla\left(u_{j-1}^{n}+4 u_{j}^{n}+u_{j+1}^{n}\right)+\frac{6 T^{2} E}{L^{2} \rho}(1+\beta \nabla)\left(-u_{j-1}^{n}+2 u_{j}^{n}-u_{j+1}^{n}\right)=0
$$

que vem a ser a equação de diferença que, numericamente, substitui a equação diferencial (2.1) que governa a propagação ondulatória em apreço.

Por outro lado, tendo-se em vista (2.8) e (2.10), o operador (2.13) ganha a seguinte redação:

$$
\nabla u_{j}^{n}=2(\cos (\omega T)-1) u_{j}^{n}
$$

onde, mediante o emprego da clássica equação de Euler, os termos complexos acabam sendo anulados.

A equação (2.14) ganha uma nova escrita, ou seja:

$$
\left(2+\cos \left(\alpha_{N} L\right)\right) \nabla u_{j}^{n}+\frac{6 T^{2} E}{L^{2} \rho}\left(1-\cos \left(\alpha_{N} L\right)\right)\left(u_{j}^{n}+\beta \nabla u_{j}^{n}\right)=0
$$


ou ainda, por força do expresso em (2.15):

$$
\left[\left(2+\cos \left(\alpha_{N} L\right)\right)(\cos (\omega T)-1)+\frac{3 T^{2} E}{L^{2} \rho}\left(1-\cos \left(\alpha_{N} L\right)\right)(1-2 \beta+2 \beta \cos (\omega T))\right] u_{j}^{n}=0
$$

que, de certa forma, consiste num problema de autovalores de dimensão um, cuja solução não trivial obtém-se sendo imposto que o termo entre colchetes seja nulo. Vale apontar que quando há mais de um único grau de liberdade por nó, tem-se uma equação característica em formato de um sistema matricial, culminando em um problema de autovalores e autovetores. Ainda, caso houvesse um maior número de pontos de integração no tempo e/ou no espaço, surgiriam novos termos cossenoidais.

\subsection{ESTUDO DA DISPERSÃO NUMÉRICA}

No sentido de facilitar o estudo em questão, é conveniente expressar a equação (2.17) em termos de parâmetros adimensionais, ou seja:

$$
\left(2+\cos \left(\alpha_{N} L\right)\right)(\cos (2 \pi \xi)-1)+3 p^{2} \xi^{2}\left(1-\cos \left(\alpha_{N} L\right)\right)(1-2 \beta+2 \beta \cos (2 \pi \xi))=0
$$

onde:

$$
\begin{array}{lll}
\mathrm{C}_{\mathrm{R}}=\sqrt{\frac{\mathrm{E}}{\rho}} \quad ; \quad \mathrm{T}_{\mathrm{R}}=\frac{2 \pi}{\omega} \quad ; \quad \lambda_{\mathrm{R}}=\mathrm{C}_{\mathrm{R}} \mathrm{T}_{\mathrm{R}} \\
\mathrm{p}=\frac{\lambda_{\mathrm{R}}}{\mathrm{L}} \quad ; \quad \xi=\frac{\mathrm{T}}{\mathrm{T}_{\mathrm{R}}}
\end{array}
$$

onde $c_{R}, T_{R}$ e $\lambda_{R}$ são respectivamente a velocidade, período e comprimento de onda analítico. Percebe-se que os parâmetros adimensionais p e $\xi$ retratam em termos adimensionais uma medida da discretização adotada; o primeiro relaciona o comprimento de onda de referência com o comprimento do elemento, e a segunda é a razão entre o passo de tempo com o período de oscilação.

Explicitando-se $\cos \left(\alpha_{N} L\right)$ por meio da equação (2.18), chega-se à expressão que define a propagação numérica no elemento, ou seja: 


$$
\cos \left(\alpha_{N} L\right)=\frac{3 p^{2}(1-2 \beta) \xi^{2}+\left(6 p^{2} \xi^{2} \beta+2\right) \cos (2 \pi \xi)-2}{3 p^{2}(1-2 \beta) \xi^{2}+\left(6 p^{2} \xi^{2} \beta-1\right) \cos (2 \pi \xi)+1}
$$

ou ainda:

$$
\eta=\frac{\alpha_{N}}{\alpha}=\frac{p}{2 \pi} \operatorname{arc} \cdot \cos \left(\frac{3 p^{2}(1-2 \beta) \xi^{2}+\left(6 p^{2} \xi^{2} \beta+2\right) \cos (2 \pi \xi)-2}{3 p^{2}(1-2 \beta) \xi^{2}+\left(6 p^{2} \xi^{2} \beta-1\right) \cos (2 \pi \xi)+1}\right)
$$

onde:

$$
\alpha=\frac{2 \pi}{\lambda_{R}}
$$

vem a ser a freqüência espacial analítica.

Como primeiro exemplo de aplicação, seja considerado o caso $\beta=1 / 4$ e $\xi=0.2$, cujo resultado acha-se ilustrado na figura 2.3a), no qual é fornecido o valor de $\eta$ em função da variável $p$. Neste gráfico, verifica-se que a curva evolui assintoticamente para o valor $\eta=1.15$ aproximadamente. Isto significa que $o$ erro referente à discretização temporal permanece com a mesma ordem de magnitude mesmo que haja um refinamento na discretização espacial. Observa-se na figura 2.3b) que a assíntota é bem mais próxima da unidade quando $\xi=0.01$. Comparando-se ambos, é visível que as formas das curvas são idênticas, verificando-se que a diferença entre elas consiste apenas numa translação. Atribui-se a essa característica o fato de que a modelagem espacial do elemento é a mesma.

É também interessante, na análise da variação de um parâmetro em função de duas variáveis, um exame do gráfico em superfície. Admitindo tanto o valor clássico $\beta=1 / 4$ quanto o modelo de diferenças centradas $(\beta=0)$, a figura 2.4 mostra o comportamento da relação $\eta$ em função de $p$ e $\xi$.

Através destas representações é possível observar que, de forma geral, a resposta numérica da freqüência espacial é maior que a teórica quando $\beta=1 / 4$, e menor quando $\beta=0$. Nesta circunstância, é instintiva a especulação de que haja um valor intermediário de $\beta$ que resulte numa melhor resposta numérica da discretização no domínio do tempo. Uma vez que o integrador espacial é o mesmo 
em ambos os casos, é esperado que qualquer curva no plano $p-\eta$ possua o mesmo formato, independente da quadratura temporal.
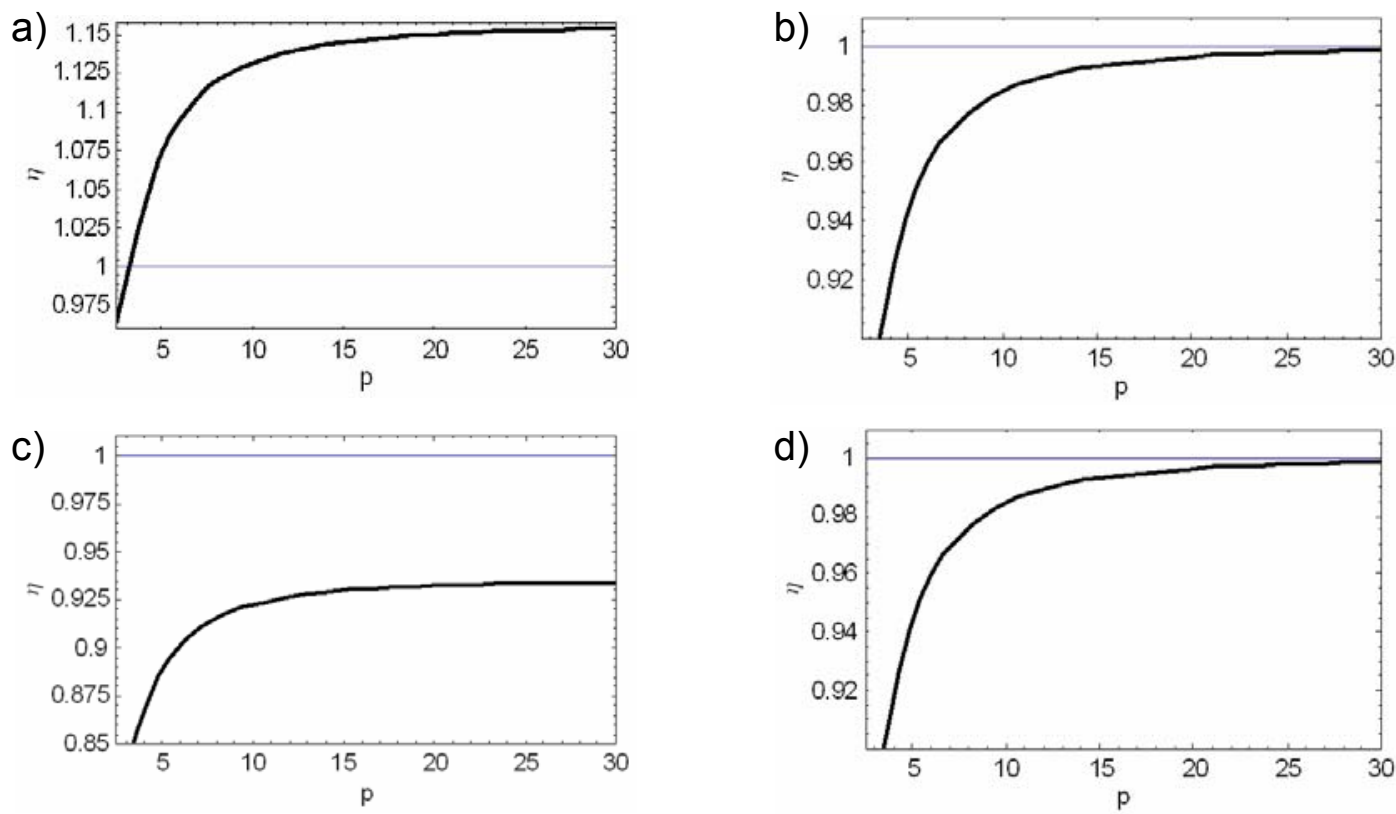

Figura 2.3 - Relação $\alpha_{N} / \alpha$ para a) $\beta=1 / 4$ e $\xi=0.2$; b) $\beta=1 / 4$ e $\xi=0.01$; c) $\beta=0$ e $\xi=0.2$ e d) $\beta=0$ e $\xi=0.01$.
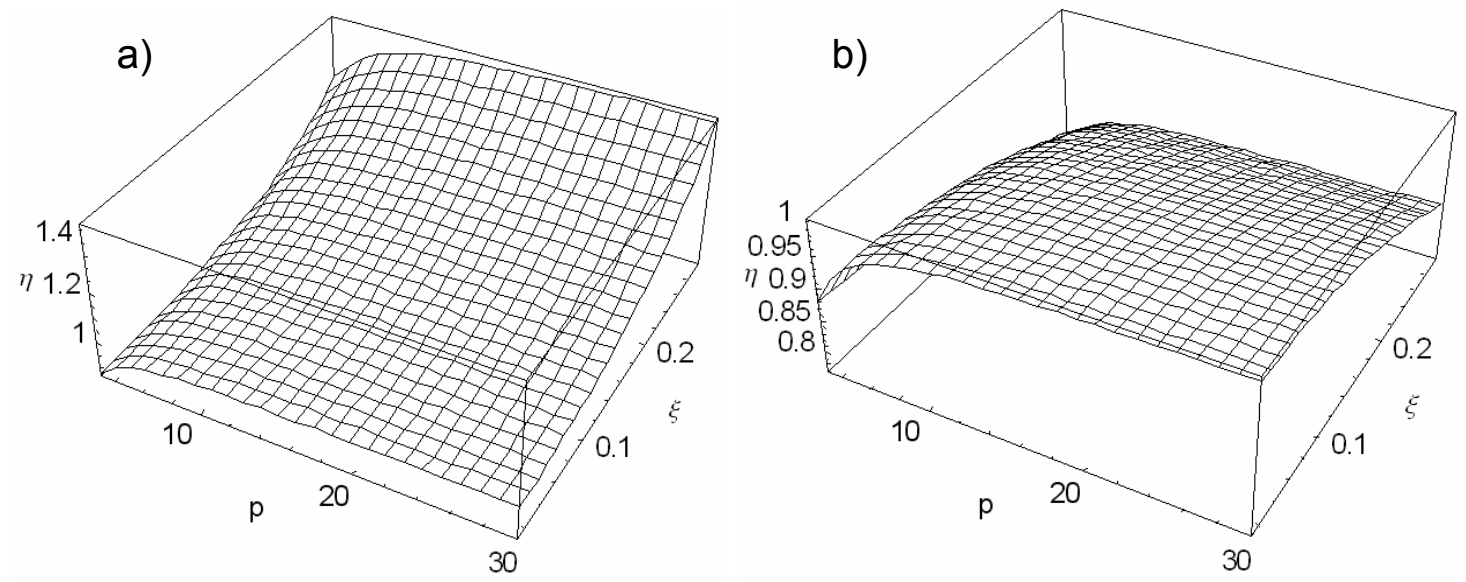

Figura $2.4-$ Relação $\alpha_{N} / \alpha$ para a) $\beta=1 / 4$ e b) $\beta=0$.

É interessante verificar o comportamento do elemento finito diante uma semi-discretização espacial, onde se considera a resposta analítica no domínio do tempo. Nesse sentido, seja considerada a equação de movimento (2.6) tendo-se em conta o expresso em (2.9), ou seja: 


$$
\frac{1}{3}\left(2+\cos \left(\alpha_{N} L\right)\right) \ddot{u}_{j}(t)+\frac{\omega^{2} p^{2}}{(2 \pi)^{2}} 2\left(1-\cos \left(\alpha_{N} L\right)\right) u_{j}(t)=0
$$

onde $\mathrm{u}_{\mathrm{j}}(\mathrm{t})$ representa o deslocamento do nó j no instante $\mathrm{t}$.

Substituindo-se na equação (2.23) o campo de deslocamentos semidiscretizado dado por:

$$
u_{j}(t)=C \cdot e^{i\left(j \alpha_{N} L+\omega t\right)}
$$

chega-se então ao valor:

$$
\eta=\frac{p}{2 \pi} \arccos \left(\frac{9 p^{2}}{3 p^{2}+2 \pi^{2}}-2\right)
$$

Por outro lado, ao se plotar a curva correspondente a (2.25), chega-se um gráfico idêntico ao mostrado na figura 2.3b. Isto pode ser evidenciado matematicamente na igualdade (2.25) ao se efetuar o limite de $\xi$ tendendo a zero.

Verificando-se então o limite de $\eta$ expresso em (2.25), para p tendendo a infinito, chega-se ao valor unitário. Todavia, aplicando o limite na igualdade (2.21), para $p$ tendendo a infinito, tem-se:

$$
\lim [\eta]_{p \rightarrow \infty}=\frac{\operatorname{sen}(\pi \xi)}{\pi \xi} \sqrt{\frac{1}{[1+2 \beta(\cos (2 \pi \xi)-1)]}}
$$

A figura 2.5 exprime o comportamento de (2.26) para dois valores de $\beta$. Matematicamente, é possível verificar que esta expressão tende à unidade quando $\xi \rightarrow 0$.

a)

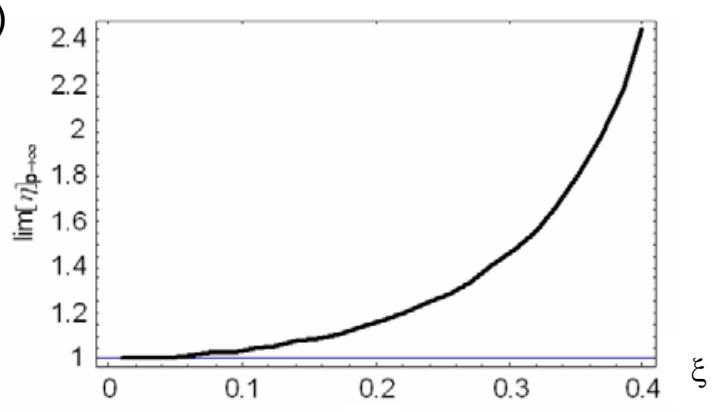

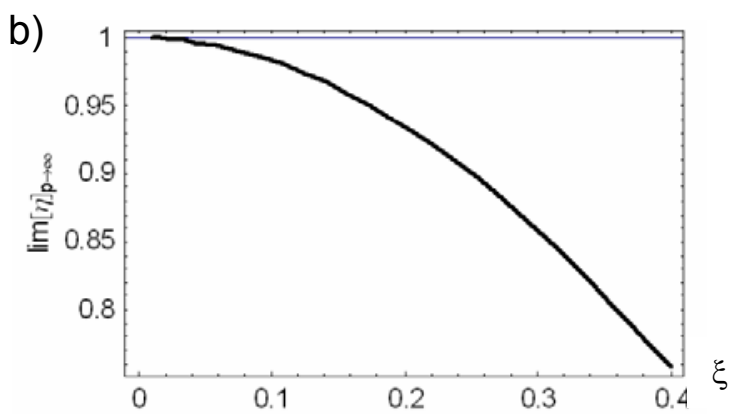

Figura 2.5 - Limite da relação $\alpha_{N} / \alpha$ para $p \rightarrow \infty$ com a) $\beta=1 / 4$ e b) $\beta=0$. 
Os estudos realizados até agora mostram o quanto a resposta numérica depende das duas parcelas distintas de erro: uma devido à discretização espacial e outra proveniente da discretização temporal.

Do ponto de vista matemático, o valor numérico da freqüência espacial $\alpha_{N}$ resultante da equação transcendente (2.20) pode assumir uma forma puramente imaginária em casos quando o segundo membro dessa igualdade tem módulo com valor maior que a unidade. De acordo com o campo de deslocamentos (2.8), isto acarreta a ocorrência de expoentes reais negativos no domínio do espaço, ou seja:

$$
u_{j}^{n}=C_{i} e^{-I m\left[\alpha_{N}\right] j L} e^{-i n \omega T}
$$

Em outras palavras, trata-se de um fenômeno de natureza puramente numérica que, pelo fato de se ter uma oscilação com decaimento exponencial ao longo do espaço, conhecido como onda evanescente, não há propriamente propagação envolvida.

Com o intuito de apontar a região do domínio onde a freqüência espacial é complexa, a figura 2.6 apresenta a imagem no eixo de Gauss de $\alpha_{N} / \alpha$.
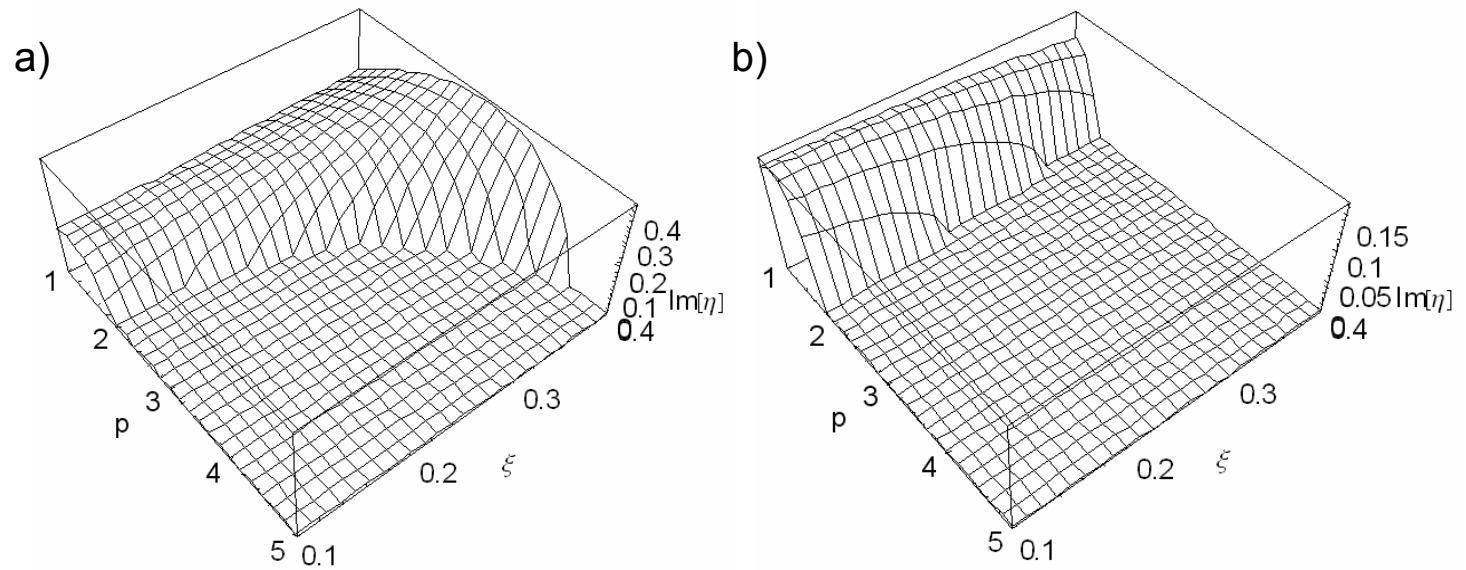

Figura $2.6-$ Imagem de $\alpha_{N} / \alpha \operatorname{com}$ a) $\beta=1 / 4$ e b) $\beta=0$.

De acordo com a equação (2.20), o domínio onde o valor da freqüência espacial assume valor real é dado por: 


$$
-1<\frac{3 p^{2}(1-2 \beta) \xi^{2}+\left(6 p^{2} \xi^{2} \beta+2\right) \cos (2 \pi \xi)-2}{3 p^{2}(1-2 \beta) \xi^{2}+\left(6 p^{2} \xi^{2} \beta-1\right) \cos (2 \pi \xi)+1}<1
$$

sendo que a solução desta inequação permite concluir que o valor limite de $p$ neste caso é dado por:

$$
\mathrm{p}_{\mathrm{Lim}}=\sqrt{\frac{1-\cos (2 \pi \xi)}{6\left(\xi^{2}-2 \xi^{2} \beta+2 \xi^{2} \beta \cos (2 \pi \xi)\right)}}
$$

Governado pela igualdade (2.25), a freqüência espacial relacionada ao caso semi-discretizado no espaço tem como limite:

$$
\mathrm{p}_{\mathrm{Lim}}=\frac{\pi}{\sqrt{3}}=1.81
$$

De fato, este valor também é obtido quando realizado o limite $\xi \rightarrow 0$ na expressão (2.29).

\subsection{EXEMPLO DE APLICAÇÃO}

O exemplo considerado neste item é o de uma barra de comprimento total $\mathrm{L}_{T}$ simplesmente engastada numa extremidade, com uma força dinâmica $F(t)=\operatorname{sen}(\omega t)$ de excitação aplicada na extremidade livre, e um número genérico $n_{e}$ de elementos finitos, conforme o esquema da figura 2.7 .

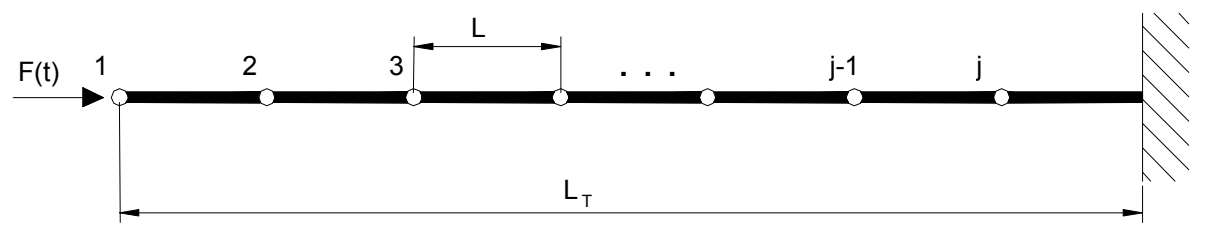

Figura 2.7 - Exemplo considerado.

Considera-se nesse exemplo de aplicação módulo de elasticidade do material altamente flexível (visando facilitar a visualização) como sendo $E=2 \mathrm{kPa}$, massa específica valendo $\rho=2500 \mathrm{~kg} / \mathrm{m}^{3}$, área da seção transversal $A=0.05 \mathrm{~m}^{2}$, e comprimento total $\mathrm{L}_{\mathrm{T}}=10 \mathrm{~m}$. 
Para o exemplo em questão a terceira freqüência angular natural analítica vale 22.2rad/s, e a sexta ale 48.9rad/s. Admitindo-se uma malha com 20 elementos $\left(n_{e}=20\right)$, incremento de tempo $T=0.02$ s, e considerando-se $\beta=1 / 4$, tem-se então a figura 2.8, o qual ilustra $u_{j}^{n}$, em metros, para freqüências convenientemente próximas dessas analíticas.

Um exame das superfícies ilustradas na figura 2.8 permite verificar que existe uma frente de onda se propaga numericamente, e que o campo de deslocamentos expresso em (2.8) é exatamente o que ocorre. Os parâmetros adimensionais para cada caso estão dispostos na tabela 2.1 .
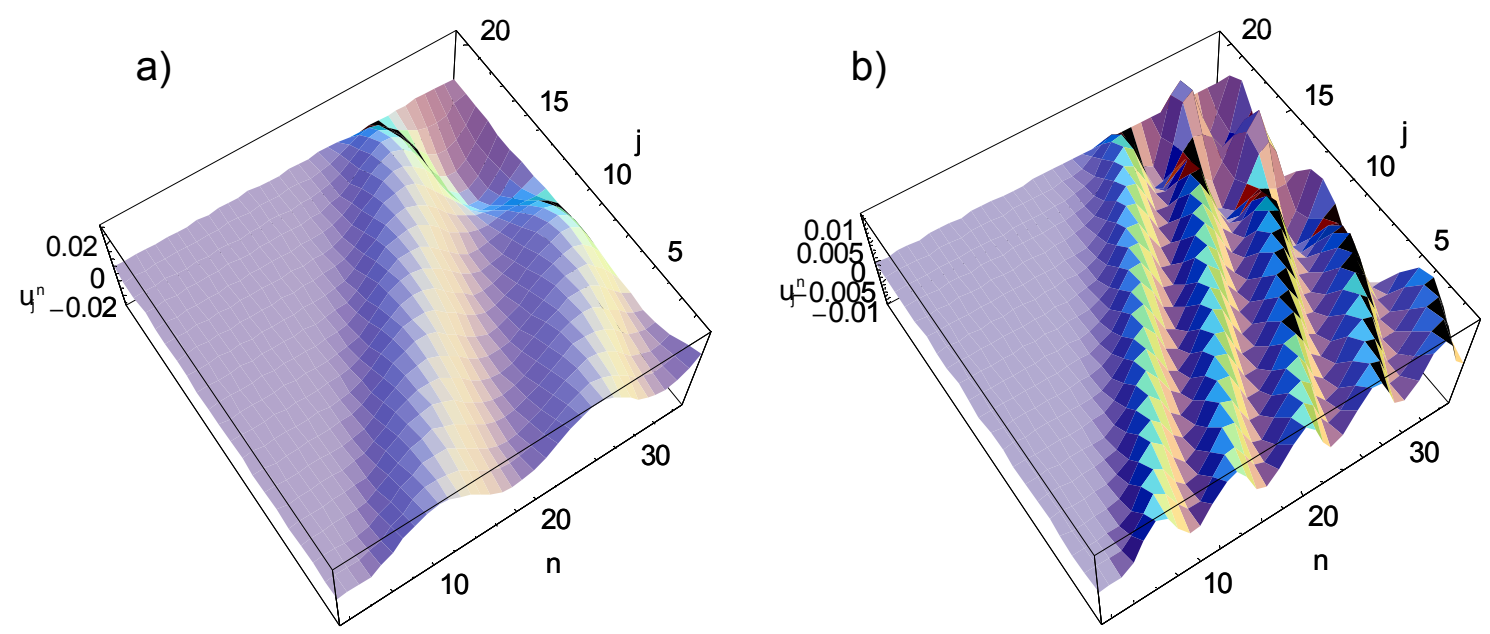

Figura 2.8 - Campo de deslocamentos $u_{j}^{n}$, em metros, para a) $\omega=22 \mathrm{rad} / \mathrm{s}$ e b) $\omega=49 \mathrm{rad} / \mathrm{s}$.

\begin{tabular}{|c|c|c|c|c|c|}
\hline Parâmetro & $\omega(\mathrm{rad} / \mathrm{s})$ & $\mathrm{T}_{\mathrm{R}}(\mathrm{s})$ & $\lambda_{\mathrm{R}}(\mathrm{m})$ & $\mathrm{p}$ & $\xi$ \\
\hline Caso a & 22 & 0.286 & 8.08 & 16.16 & 0.07 \\
\hline Caso b & 49 & 0.128 & 3.63 & 7.25 & 0.16 \\
\hline
\end{tabular}

Tabela 2.1 - Parâmetros adimensionais de cada caso.

Tendo-se em conta ser extremamente complexa a formulação de uma rotina voltada para a determinação da velocidade de propagação da frente de onda, a partir da resposta em deslocamentos de um modelo discretizado; recorrese, portanto, a uma aproximação numérica da expressão proveniente da diferenciação por partes de uma onda que se propaga no sentido positivo do eixo Ox correspondente, ou seja: 


$$
\frac{\partial u(x-c t)}{\partial t}=-c \frac{\partial u(x-c t)}{\partial x}
$$

Tendo-se em conta uma simplificada equação elementar de diferenças para correspondente ao expresso em (2.31), ou seja :

$$
c=-\left(\frac{u_{j}^{n+1}-u_{j}^{n}}{u_{j+1}{ }^{n}-u_{j}^{n}}\right) \frac{L}{T}
$$

Os resultados de (2.32), lançados nas figura 2.9a) permitem inferir que, quando a primeira derivada no espaço do deslocamento é praticamente nula, o integrador (2.32) apresenta certa perturbação. Naturalmente, para uma discretização mais refinada, como é o caso dos resultados lançados na figura $2.9 b)$, a velocidade de propagação e a aproximação (2.32) são mais precisas.
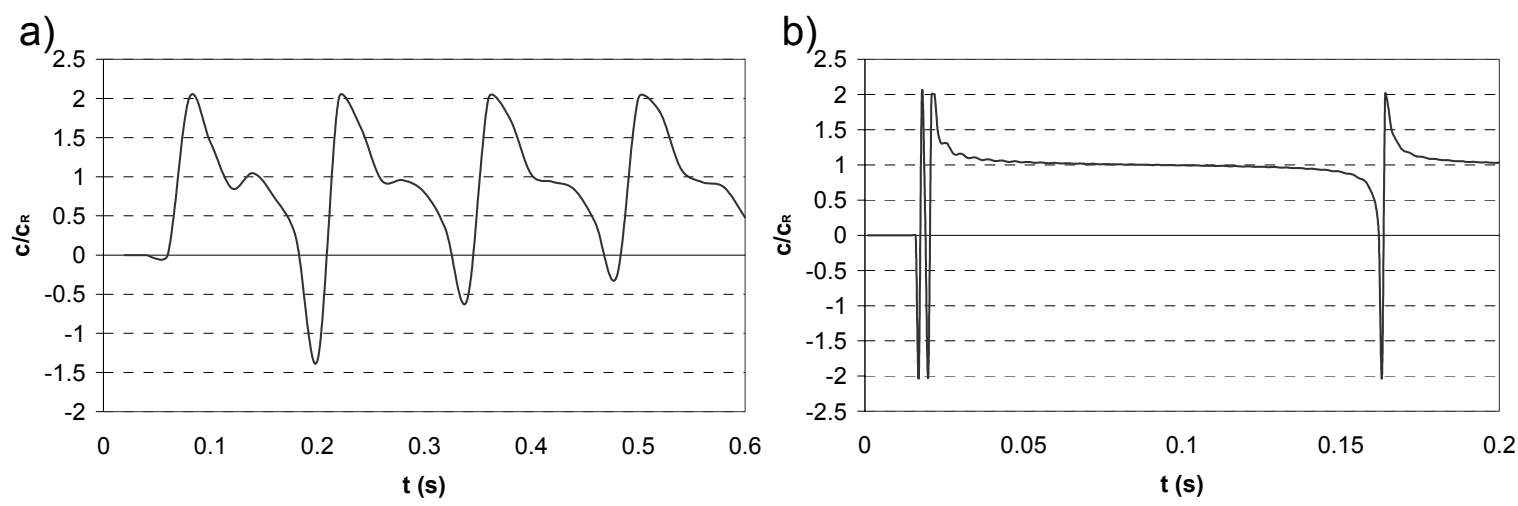

Figura 2.9 - Relação $c / c_{R}$ no nó 2 em função do tempo para o exemplo descrito, com a) $n_{e}=20$ e $T=0.02$ s e b) $n_{e}=200$ e $T=0.001 \mathrm{~s}$.

Para verificar a presença de ondas evanescentes [MULDER (1999)], seja o caso de discretizar o exemplo em questão com 25 elementos $\left(n_{e}=25\right)$, incremento de tempo valendo $T=0.001 \mathrm{~s}$ e $\beta=1 / 4$. Segundo a expressão (2.29), com o auxílio de (2.19), esta configuração tem como freqüência limite um valor de $38.79 \mathrm{~Hz}$. Desta forma, ondas com freqüência acima desta são evanescentes. Sabendo-se que o caso discretizado conta com 25 freqüências naturais, torna-se necessário filtrar o sinal de resposta em uma faixa de domínio de interesse. Portanto, serão 
consideradas duas bandas distintas, uma abaixo e outra imediatamente acima da freqüência limite. A figura 2.10 descreve a função de amplitude $H(\omega)$ de cada filtro.

O procedimento é captar os deslocamentos dos nós 1 e 24 no decorrer do tempo. Visando verificar a propagação da onda, solicita-se a barra com freqüência de $35 \mathrm{~Hz}$ com conseqüente filtragem abaixo do limite (filtro azul). Em seguida altera-se a freqüência para $45 \mathrm{~Hz}$, filtrando os sinais com a banda acima do limite (filtro verde). O resultado é exposto na figura 2.11, onde as linhas azul e verde são respectivamente o deslocamento do nó 1 e 24, respectivamente. A linha vermelha representa o deslocamento nulo. Segundo este gráfico, é notório que a onda é evanescente para o segundo caso.

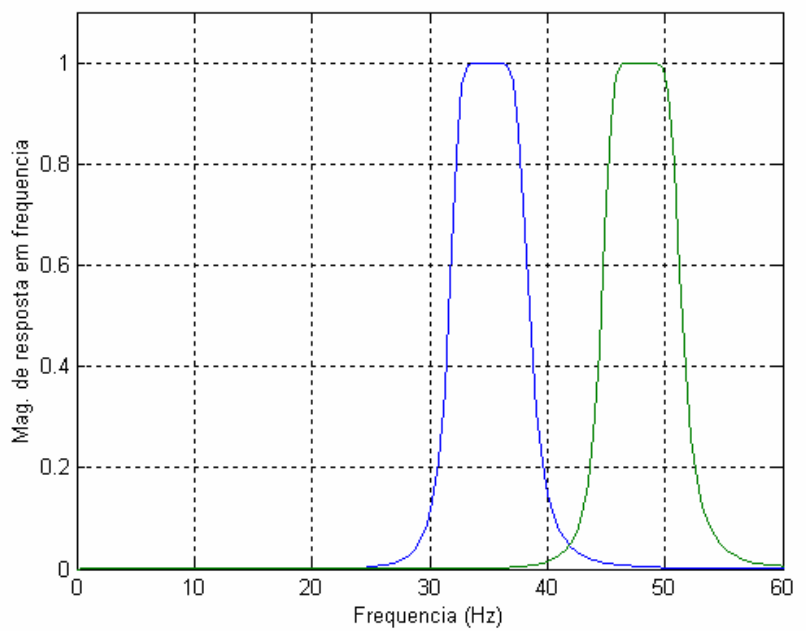

Figura 2.10 - Filtros passa-banda empregados nos cálculos.
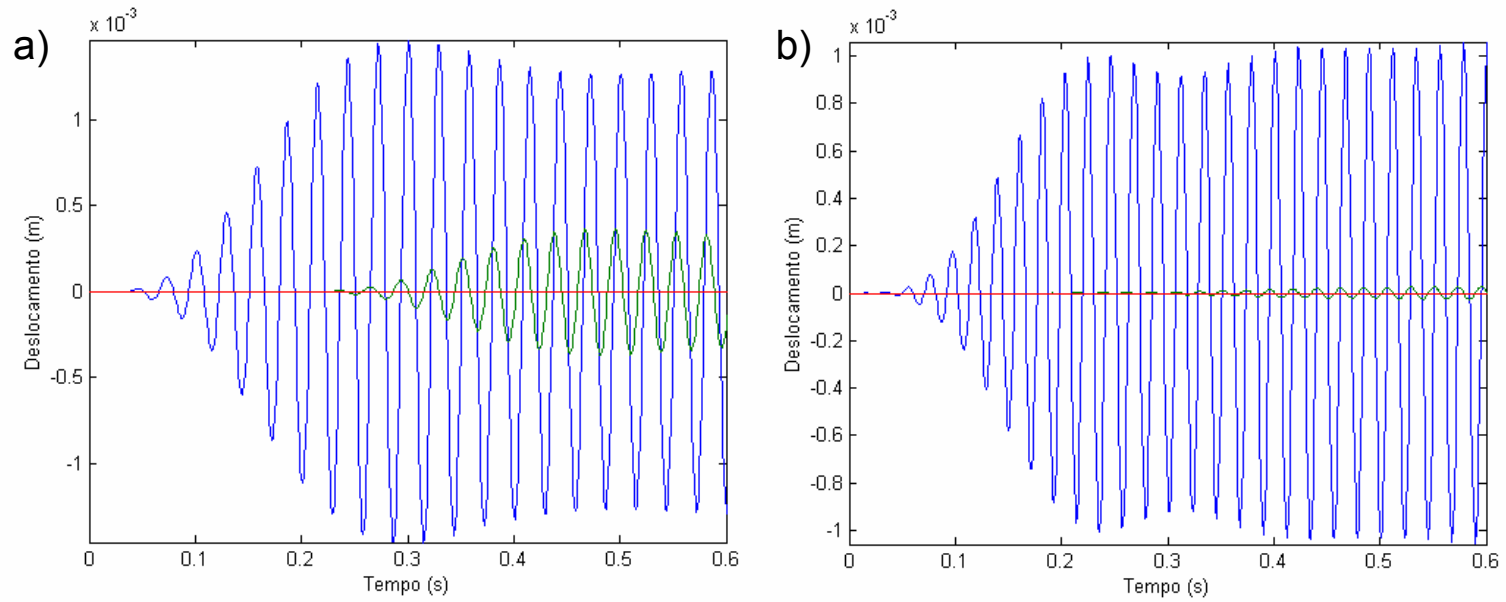

Figura 2.11 - Deslocamentos dos nós 1 e 24 (linhas azul e verde respectivamente) para a) $35 \mathrm{~Hz}$ e b) $45 \mathrm{~Hz}$. 
Do ponto de vista prático da engenharia de estruturas, o conhecimento do limite máximo permitido para a freqüência de oscilação, auxilia na escolha do tipo de elemento finito da análise, de modo a se evitar as anomalias decorrentes da participação de ondas evanescentes.

\subsection{REFLEXÕES ESPÚRIAS}

Como visto, para calcular a propagação numérica foi empregada uma malha uniforme com elementos providos de características dimensionais idênticas. Tal cenário nem sempre é possível ou viável em certos modelos estruturais reais.

Uma vez que a solução é obtida empregando-se integrações aproximadas, envolvendo equações de diferença, com velocidade de propagação que depende da dimensão da malha, é de se esperar, como de fato se verifica, que, na união de elementos de comprimento diferentes, surja reflexão de onda, dita espúria, por ocorrer apenas no problema numérico e não no analítico [JIANG (1991), BAZANT (1978)].

Para um exame mais atento dessa questão, considere-se uma barra de dimensão infinita trabalhada com a malha ilustrada na figura 2.12. A barra está dividida em duas partes semi-infinitas, uma à esquerda e outra à direita, indexadas respectivamente por 1 e 2 . Admite-se então que o comprimento $L_{1}$ de cada um dos elementos da parte esquerda seja diferente do comprimento $L_{2}$ dos elementos da parte direita. Considere-se nesta barra uma onda incidente que se propaga da esquerda para a direita. Em decorrência do equilíbrio de forças no nó j, a malha não homogênea conduz ao surgimento de uma onda espúria refletida.

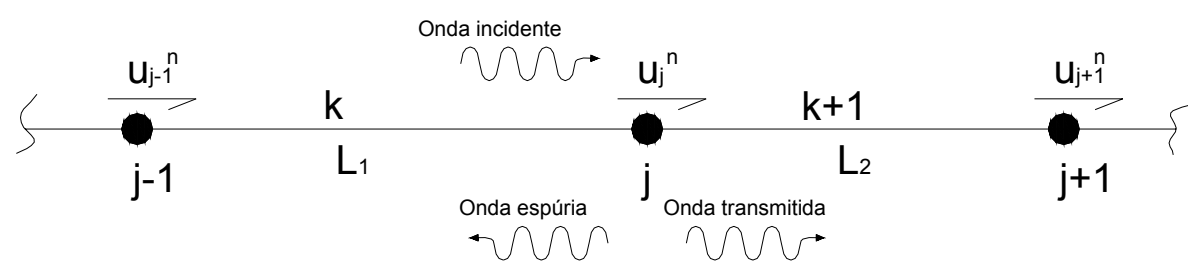

Figura 2.12 - Configuração para o cálculo da onda espúria. 
Devido à condição de interface entre os elementos, a amplitude da onda transmitida será a soma das amplitudes da onda incidente e da onda refletida, de amplitudes $\phi$ e $\kappa$ respectivamente. A parcela incidente é caracterizada pela propagação em sentido único, enquanto a parcela espúria pela propagação em dois sentidos, de forma emigrante no nó j. Assim, os vetores de deslocamentos para o elemento simples de barra são:

$$
\begin{aligned}
& \left\{u_{1}^{n}\right\}=\left\{u_{1}^{*}\right\} e^{i \omega n T}=\left[\phi\left\{\begin{array}{c}
e^{i s_{1}} \\
1
\end{array}\right\}+\kappa\left\{\begin{array}{c}
e^{-i s_{1}} \\
1
\end{array}\right\} e^{i \omega n T}\right. \\
& \left\{u_{2}^{n}\right\}=\left\{u_{2}^{*}\right\} e^{i \omega n T}=\left[\phi\left\{\begin{array}{c}
1 \\
-e^{-i s_{2}}
\end{array}\right\}+\kappa\left\{\begin{array}{c}
1 \\
e^{-i s_{2}}
\end{array}\right\}\right] e^{i \omega n T}
\end{aligned}
$$

onde s representa o ângulo $\alpha_{N} L$ e os índices 1 e 2 indicam tratar-se de grandezas relativas ao elemento da esquerda e o da direita do nó j, respectivamente.

Cumpre notar que o fenômeno da reflexão espúria manifesta-se independentemente da discretização temporal adotada. Para demonstrar isso, parte-se inicialmente para um modelo analítico no tempo e discretizado espacialmente. O modelo analítico no tempo permite relacionar a aceleração com o deslocamento na forma:

$$
\left\{\ddot{u}_{j}^{*}\right\}=-\omega^{2}\left\{u_{j}^{*}\right\}
$$

implicando-se na equação de movimento:

$$
\left(\left[\mathrm{K}_{1}^{*}\right]-\omega^{2}\left[\mathrm{M}_{1}^{*}\right]\right)\left\{\mathrm{u}_{1}^{*}\right\}+\left(\left[\mathrm{K}_{2}^{*}\right]-\omega^{2}\left[\mathrm{M}_{2}^{*}\right]\right)\left\{\mathrm{u}_{2}^{*}\right\}=0
$$

com:

$$
\begin{array}{ll}
{\left[\mathrm{K}_{1}^{*}\right]=\frac{\omega^{2} \mathrm{p}_{1}^{2}}{(2 \pi)^{2}}\left\{\begin{array}{ll}
-1 & 1
\end{array}\right\} ;} & {\left[\mathrm{K}_{2}^{*}\right]=\frac{\omega^{2} \mathrm{p}_{1}^{2}}{(2 \pi)^{2} \psi}\left\{\begin{array}{ll}
1 & -1
\end{array}\right\}} \\
{\left[\mathrm{M}_{1}^{*}\right]=\left\{\begin{array}{ll}
\frac{1}{6} & \frac{1}{3}
\end{array}\right\} \quad ; \quad\left[\mathrm{M}_{2}^{*}\right]=\psi\left\{\begin{array}{ll}
\frac{1}{3} & \frac{1}{6}
\end{array}\right\}} \\
\psi=\frac{\mathrm{L}_{2}}{\mathrm{~L}_{1}} &
\end{array}
$$


A maneira expedita de se encontrar a amplitude da onda refletida consiste em se substituir as equações (2.33) e (2.34) na (2.35). Isolando $\kappa / \phi$ na expressão assim obtida, encontra-se finalmente a relação entre a amplitude espúria a e incidente, na forma:

$$
\frac{\kappa}{\phi}=\frac{3 p_{1}^{2}\left[e^{i s_{2}}\left(1+\psi-e^{i s_{1}} \psi\right)-1\right]-2 \pi^{2} \psi\left[e^{i s_{2}}\left(e^{i s_{1}}+2 \psi+2\right)+\psi\right]}{2 \pi^{2} \psi\left\{e^{i s_{1}} \psi+e^{i s_{2}}\left[1+2 e^{i s_{1}}(1+\psi)\right]\right\}-3 p_{1}^{2}\left[e^{i s_{1}}\left(e^{i s_{2}}-1\right)+e^{i s_{2}} \psi\left(e^{i s_{1}}-1\right)\right]} e^{i s_{1}}
$$

onde $\mathrm{p}_{1}$ representa a taxa de discretização espacial dos elementos ao lado esquerdo do nó j.

Para o caso do integrador $\beta$ de Newmark, a equação de movimento é:

$$
\begin{array}{ll}
{\left[(1+2 \beta(\cos (2 \pi \xi)-1))\left[\mathrm{K}_{2}\right]+2\left[\mathrm{M}_{2}\right](\cos (2 \pi \xi)-1)\right]\left\{\mathrm{u}_{2}^{*}\right\}+\cdots} \\
\cdots+\left[(1+2 \beta(\cos (2 \pi \xi)-1))\left[\mathrm{K}_{1}\right]+2\left[\mathrm{M}_{1}\right](\cos (2 \pi \xi)-1)\right]\left\{\mathrm{u}_{1}^{*}\right\}=0 \\
{\left[\mathrm{~K}_{1}\right]=\xi^{2} \mathrm{p}_{1}^{2}\left\{\begin{array}{ll}
-1 & 1
\end{array}\right\} ;} & {\left[\mathrm{K}_{2}\right]=\frac{\xi^{2} \mathrm{p}_{1}^{2}}{\psi}\left\{\begin{array}{ll}
1 & -1
\end{array}\right\}} \\
{\left[\mathrm{M}_{1}\right]=\left\{\begin{array}{ll}
\frac{1}{6} & \frac{1}{3}
\end{array}\right\} \quad ; \quad\left[\mathrm{M}_{2}\right]=\psi\left\{\begin{array}{ll}
\frac{1}{3} & \frac{1}{6}
\end{array}\right\}}
\end{array}
$$

Sendo denominado como parcela incidente e espúria os vetores indexados com I e S, separam-se as parcelas de (2.33) na forma:

$$
u_{1, l}^{*}=\phi\left\{\begin{array}{c}
e^{i s_{1}} \\
1
\end{array}\right\} ; u_{2, I}^{*}=\phi\left\{\begin{array}{c}
1 \\
e^{-i s_{2}}
\end{array}\right\} ; u_{1, S}^{*}=\kappa\left\{\begin{array}{c}
e^{-i s_{1}} \\
1
\end{array}\right\} ; u_{2, S}^{*}=\kappa\left\{\begin{array}{c}
1 \\
e^{-i s_{2}}
\end{array}\right\}
$$

Permitindo-se escrever as relações:

$$
u_{1,1}^{*}=e^{i s_{1}}\left[\begin{array}{cc}
1 & 0 \\
0 & e^{i\left(s_{2}-s_{1}\right)}
\end{array}\right] u_{2,1}^{*} ; \quad u_{1, S}^{*}=\left[\begin{array}{cc}
0 & e^{i\left(s_{2}-s_{1}\right)} \\
1 & 0
\end{array}\right] u_{2, S}^{*}
$$

que substituídas em (2.38) retornam: 


$$
\begin{aligned}
& \left\{\begin{array}{l}
{\left[(1+2 \beta(\cos (2 \pi \xi)-1))\left[\mathrm{K}_{2}\right]+2\left[\mathrm{M}_{2}\right](\cos (2 \pi \xi)-1)\right]+\cdots} \\
\left.\cdots+\mathrm{e}^{i \mathrm{~s}_{1}}\left[(1+2 \beta(\cos (2 \pi \xi)-1))\left[\mathrm{K}_{1}\right]+2\left[\mathrm{M}_{1}\right](\cos (2 \pi \xi)-1)\right]\left[\begin{array}{cc}
1 & 0 \\
0 & \mathrm{e}^{\mathrm{i}\left(\mathrm{s}_{2}-\mathrm{s}_{1}\right)}
\end{array}\right]\right\} \mathrm{u}_{2, \mathrm{I}}^{*}+\cdots
\end{array}\right. \\
& \left\{\begin{array}{l}
{\left[(1+2 \beta(\cos (2 \pi \xi)-1))\left[\mathrm{K}_{2}\right]+2\left[\mathrm{M}_{2}\right](\cos (2 \pi \xi)-1)\right]+\cdots} \\
\cdots+\left[\left[\mathrm{K}_{2}\right] \psi+\frac{2}{\psi}\left[\mathrm{M}_{2}\right](\cos (2 \pi \xi)-1)\right]\left[\begin{array}{cc}
1 & 0 \\
0 & \mathrm{e}^{\mathrm{i}\left(\mathrm{s}_{2}-\mathrm{s}_{1}\right)}
\end{array}\right]
\end{array}\right\} \mathrm{u}_{2, \mathrm{~S}}^{*}=0
\end{aligned}
$$

graças às propriedades:

$$
\left.\left[\mathrm{K}_{2}\right]\left[\begin{array}{cc}
\mathrm{a}_{1} & 0 \\
0 & \mathrm{a}_{2}
\end{array}\right]=\frac{\left[\mathrm{K}_{1}\right.}{\psi}\right]\left[\begin{array}{cc}
0 & \mathrm{a}_{2} \\
\mathrm{a}_{1} & 0
\end{array}\right] ; \quad\left[\mathrm{M}_{2}\right]\left[\begin{array}{cc}
\mathrm{a}_{1} & 0 \\
0 & \mathrm{a}_{2}
\end{array}\right]=\psi\left[\mathrm{M}_{1}\right]\left[\begin{array}{cc}
0 & \mathrm{a}_{2} \\
\mathrm{a}_{1} & 0
\end{array}\right]
$$

com $a_{1}$ e $a_{2}$ arbitrários.

Desenvolvendo a equação (2.41) através das igualdades (2.39) conclui-se que a relação entre as amplitudes $\kappa / \phi$ passa a ser expressa por:

$$
\begin{aligned}
& \left(\mathrm{V}_{1}+\frac{\kappa}{\phi} \mathrm{V}_{2}\right) \mathrm{u}_{2, \mathrm{l}}^{*}=0 \\
& \mathrm{~V}_{1}=\left[(1+2 \beta(\cos (2 \pi \xi)-1))\left[\mathrm{K}_{2}\right]+2\left[\mathrm{M}_{2}\right](\cos (2 \pi \xi)-1)\right]+\cdots \\
& \cdots+\mathrm{e}^{\mathrm{is} 1}\left[(1+2 \beta(\cos (2 \pi \xi)-1))\left[\mathrm{K}_{1}\right]+2\left[\mathrm{M}_{1}\right](\cos (2 \pi \xi)-1)\right]\left[\begin{array}{cc}
1 & 0 \\
0 & \mathrm{e}^{\mathrm{i}\left(\mathrm{s}_{2}-\mathrm{s}_{1}\right)}
\end{array}\right] \\
& \mathrm{V}_{2}=\left[(1+2 \beta(\cos (2 \pi \xi)-1))\left[\mathrm{K}_{2}\right]+2\left[\mathrm{M}_{2}\right](\cos (2 \pi \xi)-1)\right]+\cdots \\
& \cdots+\left[(1+2 \beta(\cos (2 \pi \xi)-1))\left[\mathrm{K}_{2}\right] \psi+\frac{2}{\psi}\left[\mathrm{M}_{2}\right](\cos (2 \pi \xi)-1)\right]\left[\begin{array}{cc}
1 & 0 \\
0 & \mathrm{e}^{\mathrm{i}\left(\mathrm{s}_{2}-\mathrm{s}_{1}\right)}
\end{array}\right]
\end{aligned}
$$

Segundo (2.43a), a parcela espúria é inexistente quando $V_{1} \cdot u_{2, l}^{*}$ é nulo. De fato, efetuando tal produto tem-se:

$$
\begin{aligned}
& \mathrm{V}_{1} \mathrm{u}_{2, \mathrm{l}}^{*}=\left[(1+2 \beta(\cos (2 \pi \xi)-1))\left[\mathrm{K}_{2}\right]+2\left[\mathrm{M}_{2}\right](\cos (2 \pi \xi)-1)\right] \mathrm{u}_{2, \mathrm{I}}^{*}-\cdots \\
& \cdots+\phi\left[(1+2 \beta(\cos (2 \pi \xi)-1))\left[\mathrm{K}_{2}\right] \psi+\frac{2}{\psi}\left[\mathrm{M}_{2}\right](\cos (2 \pi \xi)-1)\right]\left\{\begin{array}{c}
1 \\
e^{\text {is }_{1}}
\end{array}\right\}
\end{aligned}
$$


A figura 2.13 é a plotagem da expressão (2.37) para alguns casos de discretização espacial, acusando-se que, por uma questão energética, a amplitude da onda refletida não pode ser superior à da onda incidente. Quando a relação assume valor unitário, presencia-se um fenômeno similar ao do tipo "wipe-out", com a diferença de que a onda refletida é evanescente, conforme exprime a figura 2.14.

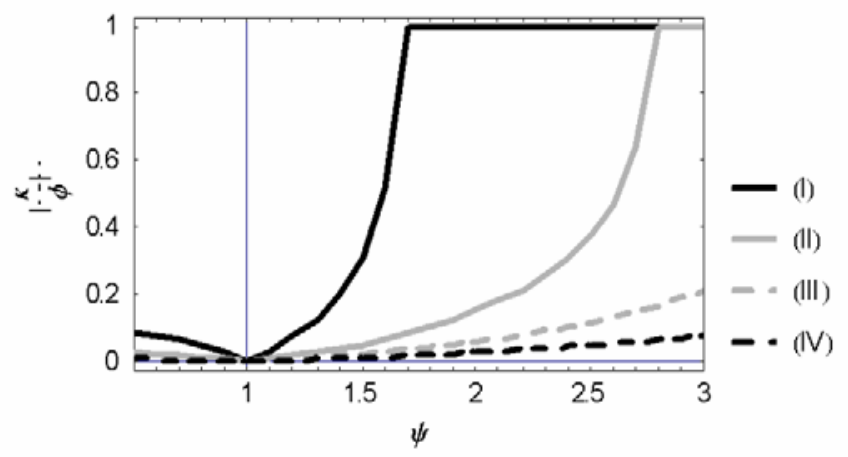

Figura 2.13 - Módulo da relação $\kappa / \phi$ do modelo semi-discretizado para I) $p_{1}=3$, II) $p_{1}=5$, III) $p_{1}=7$ e IV) $p_{1}=10$.

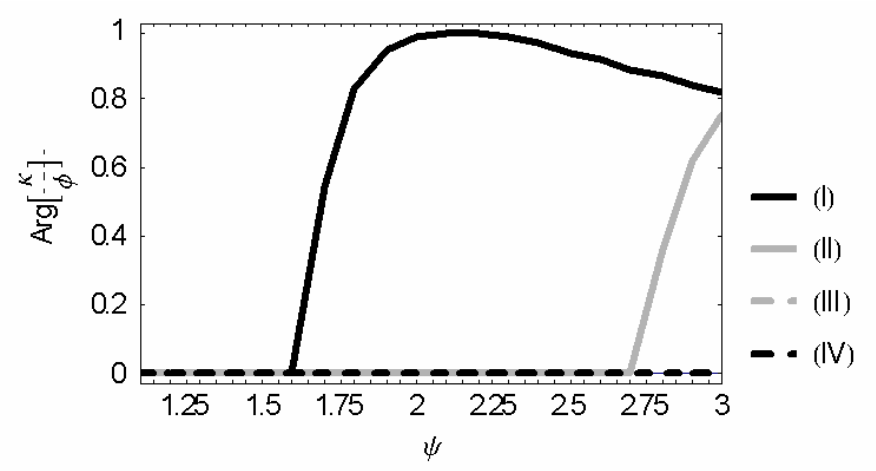

Figura 2.14 - Imagem no eixo de Gauss da relação $\kappa / \phi$ do modelo semi-discretizado para I) $p_{1}=3$, II) $\left.p_{1}=5, I I I\right) p_{1}=7$ e IV) $p_{1}=10$.

Visando evidenciar a influência do integrador temporal, a figura 2.15 ilustra o módulo $\kappa / \phi$ para alguns casos, mantendo a discretização espacial fixada em $\mathrm{p}_{1}=5$.

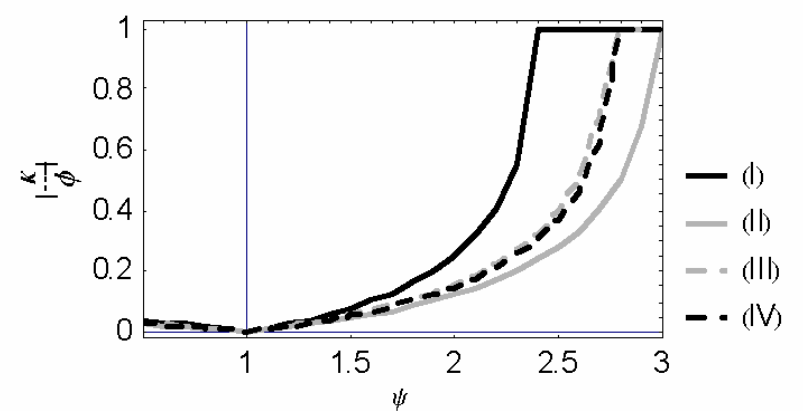

Figura 2.15 - Módulo da relação $\kappa / \phi$ do modelo discretizado para I) $\beta=1 / 4$ e $\xi=0.2$, II) $\beta=0$ e $\xi=0.2$, III) $\beta=1 / 4$ e $\xi=0.05$ e IV) $\beta=0$ e $\xi=0.05$. 
Nota-se que a tendência da quadratura temporal é, naturalmente, a resposta semi-discretizada mostrada na figura 2.15 .

No sentido de ilustrar o aparecimento de reflexão espúria, considere-se o caso de uma barra simplesmente engastada numa extremidade, como ilustra a figura 2.16, com módulo de elasticidade valendo $E=2 \mathrm{kPa}$, massa específica $\rho=2500 \mathrm{~kg} / \mathrm{m}^{3}$, área da seção transversal $A=0.05 \mathrm{~m}^{2}$ e com $6 \mathrm{~m}$ de comprimento total, sujeita a uma solicitação axial $F(t)=\operatorname{sen}(\omega t) k N$. Objetivando-se, pois, evidenciar a presença de reflexões espúrias, divide-se a barra ao meio; e discretiza-se o primeiro trecho com 4 elementos e o segundo com 2 elementos, criando-se assim uma malha não-homogênea conforme o esquema da figura 2.5

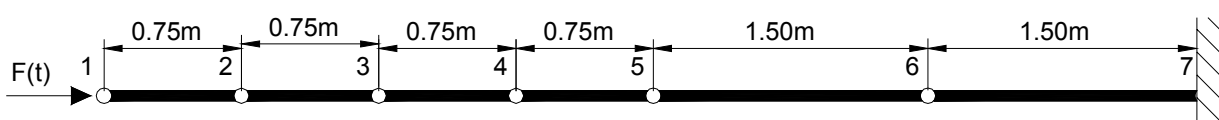

Figura 2.16 - Ilustração da malha não-homogênea.

A quarta freqüência natural analítica dessa barra vale 51.8rad/s. Portanto, empregando-se um passo de tempo de $0.025 \mathrm{~s}$, com $\beta=1 / 4$ e uma excitação com freqüência angular valendo $\omega=50 \mathrm{rad} / \mathrm{s}$, os parâmetros adimensionais correspondentes adquirem os valores $p_{1}=4.73$ e $\xi=0.20$. A figura 2.17 ilustra $o$ campo de deslocamentos $u_{j}^{n}$, em metros, nos 5 primeiros nós.

Para ilustrar a presença da reflexão espúria, a figura 2.18 é o resultado da diferença, em metros, entre o modelo homogêneo de 8 elementos iguais com o heterogêneo descrito acima.

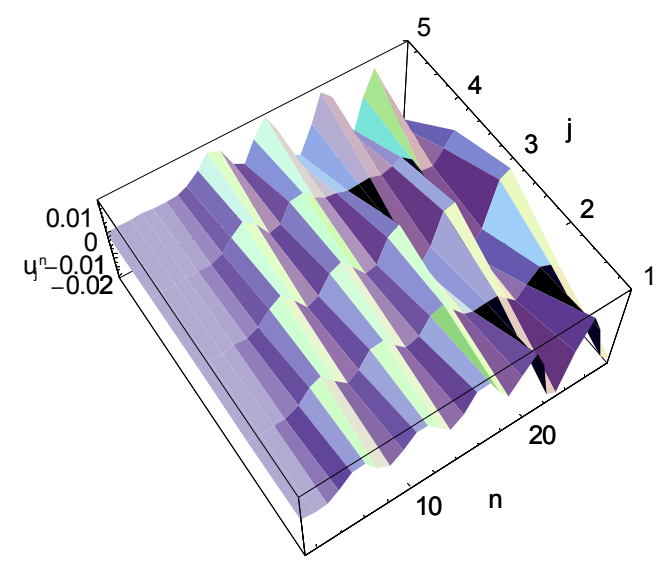

Figura 2.17 - Deslocamento $u_{j}^{n}$ nos 5 primeiros nós da malha nãohomogênea. 


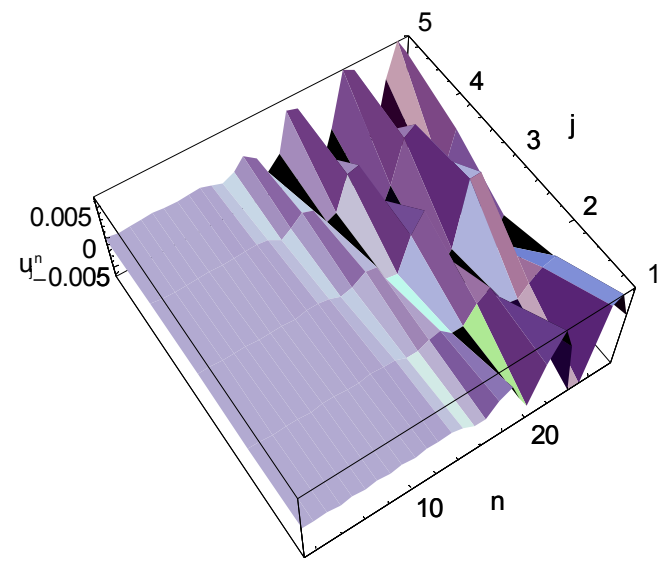

Figura 2.18 - Diferença entre o campo de deslocamentos homogêneo e heterogêneo nos 5 primeiros nós da malha.

Esta última superfície aponta que a amplitude da reflexão espúria é de aproximadamente $50 \%$ da amplitude da onda incidente. De fato, interpretando a figura 2.18, o valor de $\kappa / \phi$ para uma configuração adimensional próxima desta mostra resultado coerente com esta magnitude aí apontada. 
$\underline{\text { CAPÍtulo } 3}$

\section{Algoritmos de Integração para Elementos de Barra com Quadraturas de Ordem mais Elevada e Matriz de Massa}

\subsection{INTRODUČÃO}

O comportamento numérico de um elemento finito em análise dinâmica, conforme sabido, depende sobremaneira dos erros de truncamento de natureza local e global. O estudo dos erros de truncamento decorre da análise do desenvolvimento em série das funções envolvidas, via de regra, na forma de um polinômio de grau finito, os quais dependem do comprimento do elemento (incremento espaço) e do passo de tempo (incremento temporal). Este capítulo pretende apresentar uma metodologia voltada na busca de reduzidos erros de truncamento, objetivando conseqüente o aprimoramento das respostas obtidas. 


\subsection{COMBINAC̨ÃO OTIMIZADA}

O equilíbrio de ações em uma estrutura discretizada com vários graus de liberdade se apresenta na forma de uma equação de diferenças, conforme já discutido no capítulo precedente.

Pois bem, ao se atribuir um campo de deslocamentos exponencial, a correspondente equação de diferença passa a contemplar funções trigonométricas, como já mostrado no capítulo precedente. Por outro lado, as funções trigonométricas envolvidas podem ser expandidas em série de Taylor com truncamento, por exemplo, do tipo:

$$
\begin{aligned}
& \cos \left(\alpha_{N} L\right)=1-\frac{1}{2 !} \alpha_{N}^{2} L^{2}+\frac{1}{4 !} \alpha_{N}^{4} L^{4}-\frac{1}{6 !} \alpha_{N}^{6} L^{6}+O\left[L^{8}\right] \\
& \operatorname{sen}\left(\alpha_{N} L\right)=\alpha_{N} L-\frac{1}{3 !} \alpha_{N}^{3} L^{3}+\frac{1}{5 !} \alpha_{N}^{5} L^{5}+O\left[L^{7}\right]
\end{aligned}
$$

onde a notação $O\left(L^{k}\right)$ indica a ordem do chamado erro local, ou a ordem do primeiro termo do truncamento.

Do ponto de vista matemático, pode-se afirmar que existe uma combinação de parâmetros de discretização no espaço e no tempo (parâmetros inerentes ao método de integração aproximado), de tal sorte a se maximizar a ordem de erro. Seguindo este objetivo, é natural se esperar que os graus de liberdade do sistema passam a ser os fatores de combinação admitidos.

De fato, a expansão dos cossenos com argumentos espaciais na equação (2.17) resulta em uma aproximação da equação diferencial de propagação da onda uniaxial. Com a liberdade de se escolher os parâmetros relativos a cada termo das matrizes de rigidez e de massa, busca-se aqui investigar uma aproximação que permita zerar as parcelas de menor ordem de erro.

Por exemplo, no caso do elemento simples de barra, para o qual as matrizes de massa e de rigidez do elemento são de ordem dois (concebidas desta forma a fim de facilitar o rearranjo dos termos), quais sejam:

$$
[M]_{k}=\left[\begin{array}{ll}
m_{2} & m_{3} \\
m_{1} & m_{2}
\end{array}\right] \quad ; \quad[K]_{k}=\left[\begin{array}{ll}
k_{2} & k_{3} \\
k_{1} & k_{2}
\end{array}\right]
$$


na montagem das correspondentes matrizes do conjunto, a contribuição dos elementos (admitindo o modelo de viga infinita da figura 2.2) se dá na forma esquemática que se segue:

$$
\left[\begin{array}{ccccc}
\ddots & & & & \cdot \\
& 2 \mathrm{~m}_{2} & \mathrm{~m}_{3} & 0 & \\
& \mathrm{~m}_{1} & 2 \mathrm{~m}_{2} & \mathrm{~m}_{3} & \\
& 0 & \mathrm{~m}_{1} & 2 \mathrm{~m}_{2} & \\
\therefore & & & & \ddots
\end{array}\right]\left\{\begin{array}{c}
\vdots \\
\ddot{\mathrm{u}}_{\mathrm{j}-1} \\
\ddot{\mathrm{u}}_{\mathrm{j}} \\
\ddot{\mathrm{u}}_{\mathrm{j}+1} \\
\vdots
\end{array}\right\}+\left[\begin{array}{ccccc}
\ddots & & & & \cdot \\
& 2 \mathrm{k}_{2} & \mathrm{k}_{3} & 0 & \\
& \mathrm{k}_{1} & 2 \mathrm{k}_{2} & \mathrm{k}_{3} & \\
& 0 & \mathrm{k}_{1} & 2 \mathrm{k}_{2} & \\
\cdot & & & & \ddots
\end{array}\right]\left\{\begin{array}{c}
\vdots \\
\mathrm{u}_{\mathrm{j}-1} \\
\mathrm{u}_{\mathrm{j}} \\
\mathrm{u}_{\mathrm{j}+1} \\
\vdots
\end{array}\right\}=\{0\}
$$

O equilíbrio de forças no nó genérico j corresponde, naturalmente, à ja linha do sistema (3.3). Como pode ser visto em (3.3), embora à primeira vista conta-se com existência de quatro parâmetros a trabalhar $\left(k_{1}, k_{2}, m_{1}\right.$ e $\left.m_{2}\right)$, duas condições devem a princípio ser atendidas. A primeira delas é que a matriz de massa deve ter a soma de seus termos resultando na inércia linear total do elemento. A segunda condição é possuir determinante nulo para a matriz de rigidez.

Devido à simetria das matrizes envolvidas, os fatores que multiplicam $u_{j+1}$ são os mesmos que multiplicam $\mathrm{u}_{\mathrm{j}-1}$. Esta característica garante que um campo de deslocamentos no formato exponencial complexo, ao ser trabalhado, resulte no final numa relação trigonométrica real (a parte imaginária se anula).

A primeira indicação no sentido de se investigar uma proposta da expansão trigonométrica de ordem superior surge na integração temporal. A observação dos gráficos das figuras $2.3(\mathrm{a}, \mathrm{c})$ permite que seja inferida a existência de um valor de $\beta$ no integrador de Newmark, que minimize o erro da quadratura. Assim, tendo-se em conta (2.12), (2.13) e (2.15) a integração no tempo da equação (3.3), com o algoritmo de Newmark, se expressa:

$$
(1 / 2-\beta+\beta \cos (\omega T)) T^{2}[K]\{u\}+(\cos (\omega T)-1)[M]\{u\}=\{0\}
$$

ou ainda:

$$
[K]\{u\}+\frac{\cos (\omega T)-1}{[1 / 2-\beta+\beta \cos (\omega T)] T^{2}}[M]\{u\}=\{0\}
$$


Por outro lado, sabe-se que o problema de autovalor em questão tem a seguinte redação:

$$
[\mathrm{K}]\{\mathrm{U}\}-\omega^{2}[\mathrm{M}]\{\mathrm{u}\}=\{0\}
$$

Assim, examinando-se a expansão em série do fator de (3.5), ou seja:

$$
\frac{\cos (\omega \mathrm{T})-1}{[1 / 2-\beta+\beta \cos (\omega \mathrm{T})]^{2}}=-\omega^{2}+\frac{\omega^{4}(1-12 \beta)}{12} \mathrm{~T}^{2}+\mathrm{O}\left[\mathrm{T}^{4}\right]
$$

tem-se que o erro global do operador trapezoidal $(\beta=1 / 4)$, como sabido, é de segunda ordem.

Nota-se que, qualquer que seja o elemento finito considerado, o integrador temporal de Newmark de maior ordem de erro ocorre para $\beta=1 / 12$, com erro global de quarta ordem. Obviamente, o sistema ainda contém uma margem de erro atribuída à integração espacial.

O método proposto visa aplicar esta mesma expansão na discretização espacial, buscando um integrador com menor erro em relação ao parâmetro $L$ (dimensão do elemento).

Para a barra infinita discretizada conforme a figura 2.2, sujeita a um campo de deslocamentos de acordo com a igualdade (2.8), é pretendido combinar os fatores dos deslocamentos dos nós j-1, j e j+1 de forma generalizada (envolvendo matrizes em princípio não necessariamente simétricas). Assim, a equação de movimento no nó j pode ser admitida como sendo uma combinação do tipo:

$$
\frac{m}{\nabla} \ddot{u}_{j}^{*}+\stackrel{k}{\nabla} u_{j}^{*}=0
$$

onde:

$$
\begin{aligned}
& \frac{\mathrm{q}}{\nabla} \mathrm{u}_{\mathrm{j}}^{*}=\mathrm{q}_{1} \mathrm{u}_{\mathrm{j}-1}^{*}+2 \mathrm{q}_{2} \mathrm{u}_{\mathrm{j}}^{*}+\mathrm{q}_{3} \mathrm{u}_{\mathrm{j}+1}^{*} \\
& \mathrm{u}_{1}^{*}=\mathrm{u}_{1}^{\mathrm{n}} \mathrm{e}^{\mathrm{i}(2 \pi \xi) \mathrm{n}}
\end{aligned}
$$

sendo $q_{i}$ um parâmetro de combinação. 
Tomando-se a expressão (2.8), tendo-se em conta o expresso em (2.9), tem-se que o equilíbrio do nó genérico j passa a ter a seguinte redação:

$$
\ddot{u}_{j}^{*}+\left(\frac{k_{1} e^{-\alpha_{N} L}+2 k_{2}+k_{3} e^{\alpha_{N} L}}{m_{1} e^{-\alpha_{N} L}+2 m_{2}+m_{3} e^{\alpha_{N} L}}\right) u_{j}^{*}=0
$$

Por outro lado, expandindo em séries de Taylor os termos exponenciais presentes em (3.10), chega-se a um polinômio em termos do incremento $L$ na forma:

$$
\begin{aligned}
& \frac{k_{1} e^{-a_{N} L}+2 k_{2}+k_{3} e^{\alpha_{N} L}}{m_{1} e^{-a_{N} L}+2 m_{2}+m_{3} e^{\alpha_{N} L}}=\frac{k_{1}+2 k_{2}+k_{3}}{m_{1}+2 m_{2}+m_{3}}+2 i \frac{k_{3}\left(m_{1}+m_{2}\right)+k_{2}\left(m_{1}-m_{3}\right)-k_{1}\left(m_{2}+m_{3}\right)}{\left(m_{1}+2 m_{2}+m_{3}\right)^{2}} \alpha_{N} L+\cdots \\
& \cdots+\frac{k_{1}\left[\left(m_{1}-2 m_{2}\right) m_{2}+\left(2 m_{1}-3 m_{2}\right) m_{3}-2 m_{3}^{2}\right]-k_{2}\left[m_{1}^{2}+m_{3}\left(m_{3}-2 m_{2}\right)-2 m_{1}\left(m_{2}+3 m_{3}\right)\right]+k_{3}\left[-2 m_{1}^{2}-3 m_{1} m_{2}-2 m_{2}^{2}+\left(2 m_{1}+m_{2}\right) m_{3}\right]}{\left(m_{1}+2 m_{2}+m_{3}\right)^{3}} \alpha_{N}^{2} L^{2}+O\left[L^{3}\right]
\end{aligned}
$$

Tendo-se agora em conta que a equação diferencial que governa o equilíbrio (2.1) pode também ser expressa como:

$$
\ddot{u}(x, t)+c_{R}^{2} \alpha^{2} u(x, t)=0
$$

resta então buscar maximizar a ordem do erro global na expansão (3.11) face ao confronto de (3.10) com (3.12).

Nesse sentido, resolvendo sistema de equações algébricas não lineares resultante, conclui-se que para:

$$
\begin{array}{ll}
\mathrm{m}_{1}=\mathrm{m}_{3} \quad ; & \mathrm{m}_{2}=5 \mathrm{~m}_{3} \\
\mathrm{k}_{1}=\mathrm{k}_{3}=-\frac{12 \mathrm{c}_{\mathrm{R}}^{2}}{\mathrm{~L}^{2}} \mathrm{~m}_{3} ; & \mathrm{k}_{2}=\frac{12 \mathrm{c}_{\mathrm{R}}^{2}}{\mathrm{~L}^{2}} \mathrm{~m}_{3}
\end{array}
$$

a equação (3.10) adquire a redação:

$$
\ddot{u}_{j}^{*}+C_{R}^{2}\left(\alpha_{N}^{2}-\frac{\alpha_{N}^{6} L^{4}}{240}+O\left[L^{6}\right]\right) u_{j}^{*}=0
$$

Esta última equação indica que, para esta combinação, a propagação numérica está vinculada a um erro global de quarta ordem em relação ao seu comprimento L. Teoricamente, seria como dito, em princípio, admissível na 
expressão (3.10) que as matrizes envolvidas não fossem necessariamente simétricas, porém, a maximização da ordem do erro global na resposta, implica em $m_{1}=m_{3}$ e $k_{1}=k_{3}$ resultando-se, pois, em matrizes de massa e de rigidez simétricas.

Tendo-se em vista o expresso em (3.2) e (3.13), pode-se afirmar que a combinação otimizada em relação à freqüência espacial é:

$$
[M]_{k}=\left[\begin{array}{cc}
5 & 1 \\
1 & 5
\end{array}\right] \mathrm{m}_{3} \quad ; \quad[\mathrm{K}]_{\mathrm{k}}=\frac{12 \mathrm{c}_{\mathrm{R}}^{2}}{\mathrm{~L}^{2}}\left[\begin{array}{cc}
1 & -1 \\
-1 & 1
\end{array}\right] \mathrm{m}_{3}
$$

A matriz de rigidez apresentada em (3.16) possui determinante nulo como deveria ser esperado; bem como a matriz de massa deve ter como somatório de seus termos o valor da inércia linear do elemento, ou seja:

$$
12 \mathrm{~m}_{3}=\rho \mathrm{AL}
$$

Esta condição de contorno é atendida quando $m_{3}=\rho A L / 12$, implicando finalmente nas matrizes:

$$
[\mathrm{M}]_{\mathrm{k}}=\frac{\rho \mathrm{AL}}{12}\left[\begin{array}{ll}
5 & 1 \\
1 & 5
\end{array}\right] \quad ; \quad[\mathrm{K}]_{\mathrm{k}}=\frac{\mathrm{EA}}{\mathrm{L}}\left[\begin{array}{cc}
1 & -1 \\
-1 & 1
\end{array}\right]
$$

que apresentam elevada precisão numérica.

\subsection{INTEGRADOR ESPACIAL PROPOSTO}

O integrador espacial proposto neste trabalho emprega exatamente o mesmo procedimento adotado na quadratura temporal de Newmark, resumida na equação (2.12), o qual utiliza três pontos discretos no tempo para aproximar a aceleração.

Pretende-se agora estudar o comportamento numérico desse integrador espacial inspirado no método de Newmark, aproximando-se a derivada segunda no espaço segundo o expresso em (2.12), ou seja:

$$
\nabla_{s} u_{j}^{n}=L^{2}\left(1+\beta_{S} \nabla_{S}\right) u^{n}{ }_{j}^{n}
$$


onde $\beta_{S}$ é o ponderador do integrador espacial e $\nabla_{S}$ é um operador que resume:

$$
\nabla_{s} u_{j}^{n}=u_{j-1}^{n}-2 u_{j}^{n}+u_{j+1}^{n}
$$

válido para a variável espaço.

Admitindo-se o formato exponencial do campo de deslocamentos, a exemplo de (2.8), obtém-se então uma relação similar à (2.15), qual seja:

$$
\nabla_{S} u_{j}^{n}=2\left(\cos \left(\alpha_{N} L\right)-1\right) u_{j}^{n}
$$

Aplicando-se, pois, este integrador, a equação de movimento passa a ter redação similar à (3.5):

$$
\ddot{u}_{j}^{n}-\frac{2 c_{R}^{2}\left(\cos \left(\alpha_{N} L\right)-1\right)}{L^{2}\left[1+2 \beta_{S}\left(\cos \left(\alpha_{N} L\right)-1\right)\right]} u_{j}^{n}=0
$$

Finalmente, realizando a expansão polinomial dos cossenos envolvidos nesta equação tem-se:

$$
-\frac{2 c_{R}^{2}\left(\cos \left(\alpha_{N} L\right)-1\right)}{L^{2}\left[1+2 \beta_{S}\left(\cos \left(\alpha_{N} L\right)-1\right)\right]}=c_{R}^{2} \alpha_{N}^{2}+\frac{12 \beta_{S}-1}{12} c_{R}^{2} L^{2} \alpha_{N}^{4}+O\left[L^{4}\right]
$$

Assim como no caso temporal, a integração espacial com maior ordem de erro ocorre para $\beta_{S}=1 / 12$. Efetuando a conversão da equação global de diferenças a um sistema matricial local correspondente, assim como foi feito no tópico anterior, chega-se às matrizes de massa e de rigidez:

$$
[\mathrm{M}]_{\mathrm{k}}=\rho \mathrm{AL}\left[\begin{array}{cc}
\frac{1}{2}-\beta_{\mathrm{S}} & \beta_{\mathrm{S}} \\
\beta_{\mathrm{S}} & \frac{1}{2}-\beta_{\mathrm{S}}
\end{array}\right] \quad ; \quad[\mathrm{K}]_{\mathrm{K}}=\frac{\mathrm{EA}}{\mathrm{L}}\left[\begin{array}{cc}
1 & -1 \\
-1 & 1
\end{array}\right]
$$

Observa-se nestas matrizes que $\beta_{S}=1 / 6$ corresponde ao modelo clássico de massa consistente, $\beta_{S}=1 / 12$ ao modelo de ordem de erro maximizada e $\beta_{S}=0$ ao modelo de massa concentrada [SCHREYER (1978)]. Inspirado no valor incondicionalmente estável de $\beta$ de Newmark, um quarto modelo de elemento pode ser admitido fazendo $\beta_{S}=1 / 4$. 
Duas considerações podem ser ressaltadas em relação a este último modelo. A primeira delas é que, quando efetuado o cálculo da propagação numérica neste tipo de elemento, conclui-se que a onda nunca é evanescente porque $\eta$ (relação entre o valor numérico e o teórico da freqüência espacial) é sempre real. Outro apontamento relevante é o fato de este modelo não apresentar ondas espúrias em malhas não homogêneas.

À título de implementação nessa busca de integradores de ordem mais elevada, uma outra vertente sugestiva consiste no emprego de operadores de diferenças finitas do tipo hermitiano, que tem como fundamento uma combinação de valores discretos da função a ser integrada e de suas derivadas, de tal sorte que a expansão via séries de Taylor tenha anulado o maior número possível de parcelas no truncamento. As combinações hermitianas referentes ao integrador espacial seriam análogas ao temporal, apresentando-se, por exemplo, na seguinte escrita:

$$
\begin{aligned}
& 12 u_{j}^{n}-12 u_{j+1}^{n}+6 L u_{j}^{\prime n}+6 L u_{j+1}^{\prime n}+L^{2} u_{j}^{\prime n}-L^{2} u_{j+1}^{\prime n}=0+O\left[L^{5}\right] \\
& 12 u_{j-1}^{n}-12 u_{j}^{n}+6 L u_{j-1}^{\prime n}+6 L u_{j}^{\prime n}+L^{2} u_{j-1}^{\prime n}-L^{2} u_{j}^{\prime n}=0+O\left[L^{5}\right]
\end{aligned}
$$

onde, a exemplo de (2.11), não se acham envolvidas derivadas de terceira ordem.

As igualdades narradas em (3.24) podem ser verificadas com o desenvolvimento das funções presentes no primeiro membro em séries de Taylor. Subtraindo-se membro a membro as equações (3.24), tem-se:

$$
6 L u_{j+1}^{\prime n}-6 L u_{j-1}^{\prime n}=L^{2} \nabla u_{j}^{\prime n}+12 \nabla u_{j}^{n}+O\left[L^{5}\right]
$$

onde o operador $\nabla$ é similar ao descrito na expressão (2.13), porém combinandose agora termos segundo o espaço, ou seja:

$$
\nabla u_{j}^{n}=u_{j-1}^{n}-2 u_{j}^{n}+u_{j+1}^{n}
$$

onde o índice j refere-se à estação da variável espaço. 
Analogamente, a deformação $u^{\prime}$ pode ser eliminada, derivando-se e multiplicando-se por $L$ as igualdades (3.24), com conseqüente adição destas duas expressões resultantes, obtendo-se:

$$
\begin{aligned}
& 6 L u_{j+1}^{\prime n}-6 L u_{j-1}^{\prime n}=3 L^{2} \bar{\nabla} u_{j}^{\prime n}+O\left[L^{3}\right] \\
& \bar{\nabla} u_{j}^{n}=u_{j-1}^{n}+2 u_{j}^{n}+u_{j+1}^{n}
\end{aligned}
$$

cuja substituição em (3.25) resulta:

$$
\nabla u_{j}^{n}=\frac{L^{2}}{12}(3 \bar{\nabla}-\nabla) u_{j}^{\prime n}+O\left[L^{3}\right]
$$

Aplicando a operação $\frac{L^{2}}{12}(3 \bar{\nabla}-\nabla)$ na equação de movimento, chega-se finalmente a:

$$
(3 \bar{\nabla}-\nabla) \ddot{u}_{j}^{n}-12 \frac{C_{R}^{2}}{L^{2}} \nabla u_{j}^{n}=0
$$

Implicando-se na equação de diferenças:

$$
\left\{\begin{array}{lll}
\frac{1}{6} & \frac{2}{3} & \frac{1}{6}
\end{array}\right\}\left\{\ddot{u}^{n}\right\}+\frac{c_{R}^{2}}{L^{2}}\left\{\begin{array}{lll}
-1 & 2 & -1
\end{array}\right\}\left\{u^{n}\right\}=0
$$

onde:

$$
\left\{u^{n}\right\}^{\top}=\left\{\begin{array}{lll}
u_{j-1}^{n} & u_{j}^{n} & u_{j+1}^{n}
\end{array}\right\}
$$

empregando-se, pois, notação vetorial clássica.

Realizando-se as operações matriciais, obtêm-se as mesmas matrizes de massa e de rigidez relativas ao modelo consistente clássico, descritas segundo o expresso em (2.5).

É interessante recordar que a equação de movimento, assim como disposta em (3.29), está associada a um truncamento de terceira ordem no domínio do espaço. Isto indica que um refinamento na integração através da variável deslocamentos (assim como o hermitiano), não implica em uma resposta satisfatória em termos de freqüência e da freqüência espacial. 


\subsection{RESULTADOS}

As figuras 3.1 e 3.2 ilustram o valor real da razão $\eta$ em função respectivamente de $\xi$ e de $p$ para os casos: I) $\beta_{S}=1 / 4$; II) $\beta_{S}=1 / 6$ (modelo consistente clássico); III) $\beta_{S}=1 / 12$ (modelo de ordem elevada) e IV) $\beta_{S}=0$ (modelo de massa concentrada), fixando-se que $p=5$ na figura 3.1 e que $\xi=0.2$ no seguinte. A figura 3.3 delimita e quantifica a zona evanescente do domínio considerado através da plotagem da parcela imaginária de $\eta$ em função de $p$ e $\xi$ para vários casos de discretização.

a)

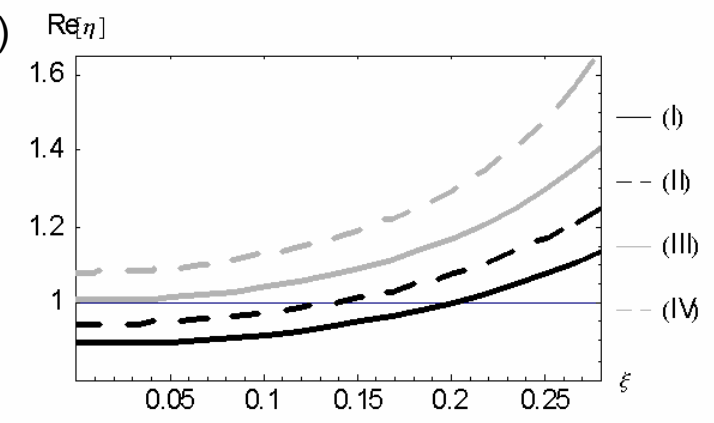

b)

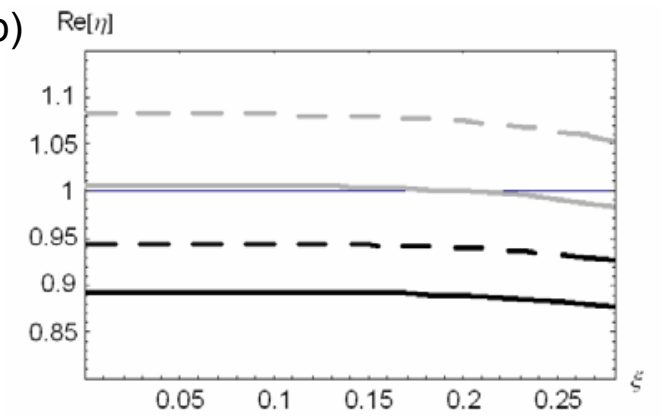

Figura 3.1 - Valor real de $\eta$ em função de $\xi$ com $p=5$ para a integração temporal de Newmark com a) $\beta=1 / 4$ (modelo incondicionalmente estável de Newmark) e b) $\beta=1 / 12$ (modelo de ordem elevada).

a)

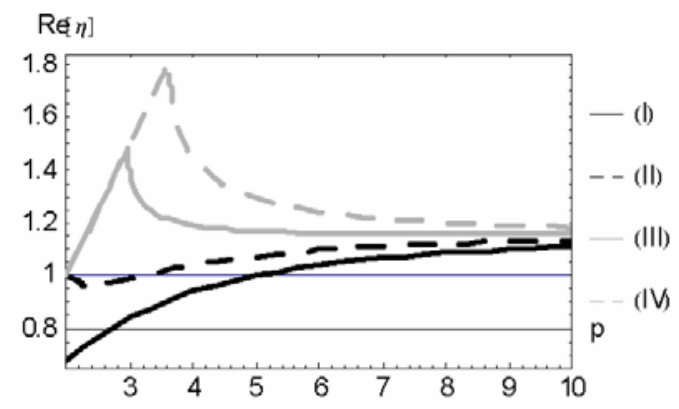

b)

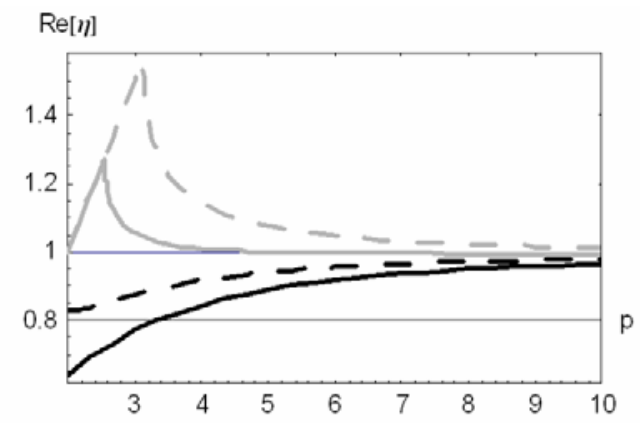

Figura 3.2 - Valor real de $\eta$ em função de $p \operatorname{com} \xi=0.2$ para a integração de Newmark com a) $\beta=1 / 4$ e b) $\beta=1 / 12$.

Observa-se na figura 3.1, que a forma da curva é ditada pelo integrador temporal e seu cruzamento no eixo das ordenadas é regido pela quadratura numérica espacial. A figura 3.2 indica que o erro associado ao parâmetro $\beta$ de Newmark igual a $1 / 4$ é maior que no caso onde $\beta=1 / 12$, conforme o previsto. 
a)

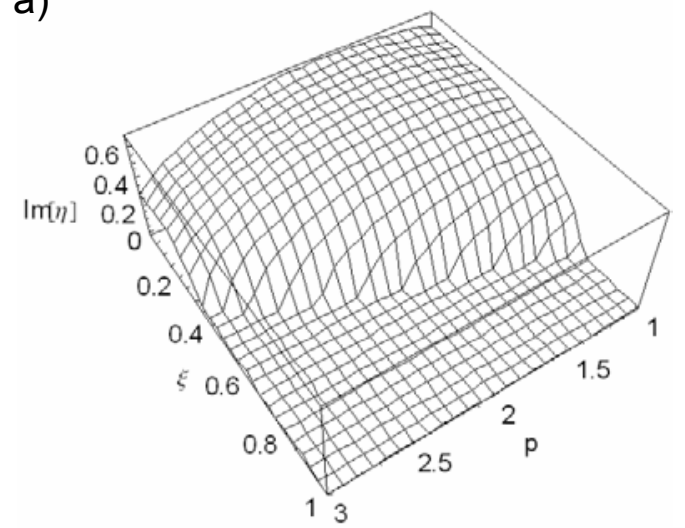

c)

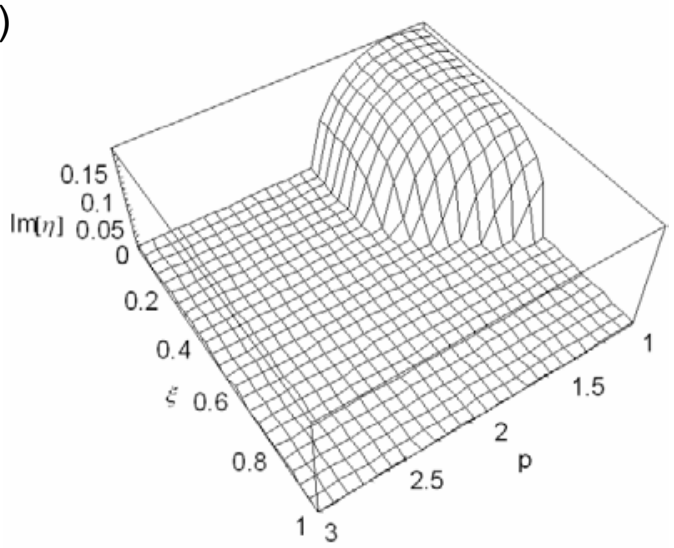

b)

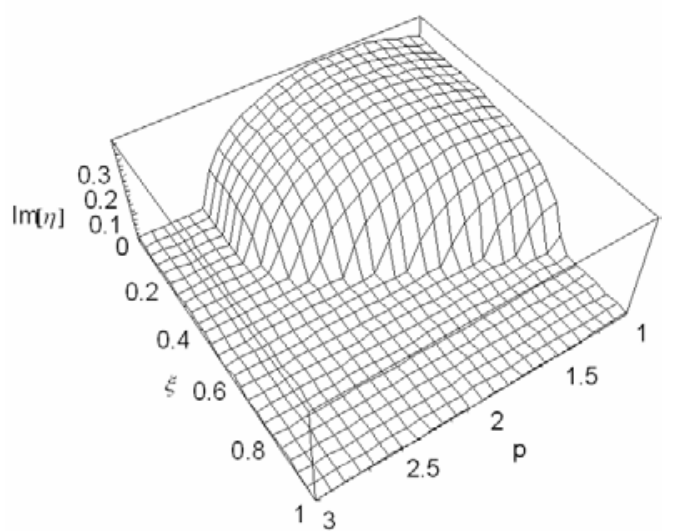

d)

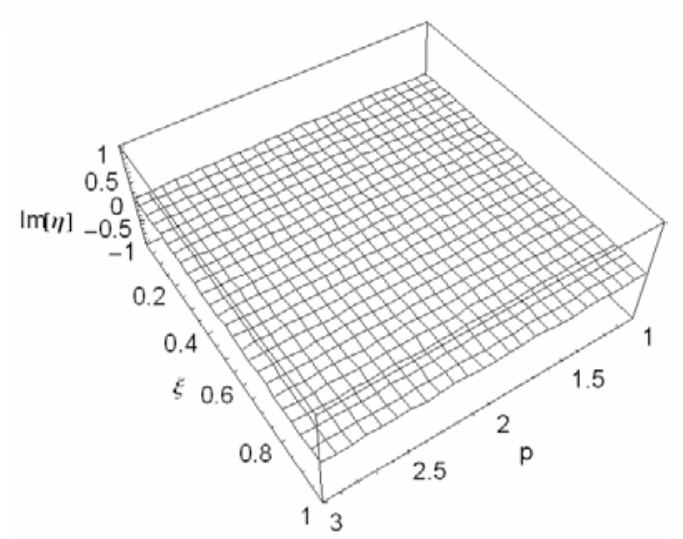

Figura 3.3 - Parcela imaginária de $\eta$ em função de $p$ e $\xi$ para a integração temporal de Newmark com $\beta=1 / 12$ e integração espacial com a) $\beta_{S}=0$, b) $\beta_{S}=1 / 12$, c) $\beta_{S}=1 / 6$ e d) $\beta_{S}=1 / 4$.

Nota-se na figura 3.3 que a região e a magnitude do domínio da onda evanescente decresce com o conforme aumenta-se do parâmetro $\beta_{\mathrm{S}}$, atingindo-se seu limite no valor $1 / 4$, onde não há a presença de onda evanescente.

Tomando-se uma semi-discretização espacial, a figura 3.4 expõe o módulo da relação entre a amplitude espúria e a incidente em função da relação da dimensão dos elementos concorrendo no nó $L_{2} / L_{1}$ para os quatro modelos descritos no último caso. O gráfico seguinte mostra o valor da parcela imaginária para essa relação no caso $p_{1}=5$, acusando-se reflexão espúria evanescente.

Percebe-se que a intensidade espúria está vinculada à estabilidade (bifurcação) numérica relativa ao fenômeno evanescente, uma vez que elementos mais suscetíveis às ondas evanescentes possuem amplitude espúria mais elevada. 

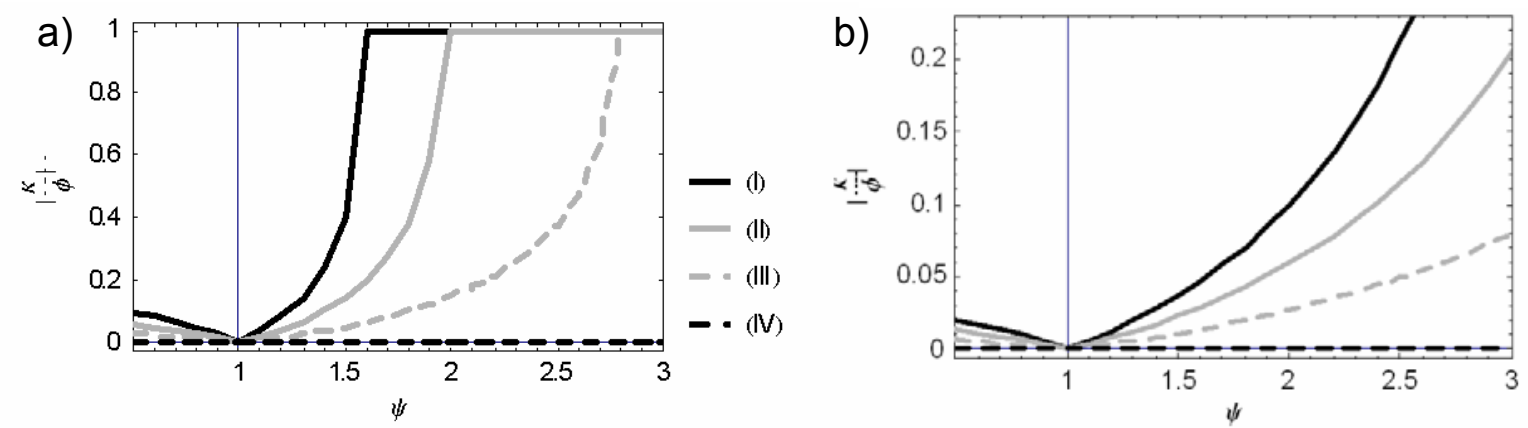

Figura 3.4 - Módulo da relação $\kappa / \phi$ em função de $\psi$ na semi-discretização analítica no domínio do tempo e integração espacial com I) $\beta_{S}=0$, II) $\beta_{S}=1 / 12$, III) $\beta_{S}=1 / 6$ e IV) $\beta_{S}=1 / 4$ para a) $p_{1}=5$ e b) $p_{1}=10$.

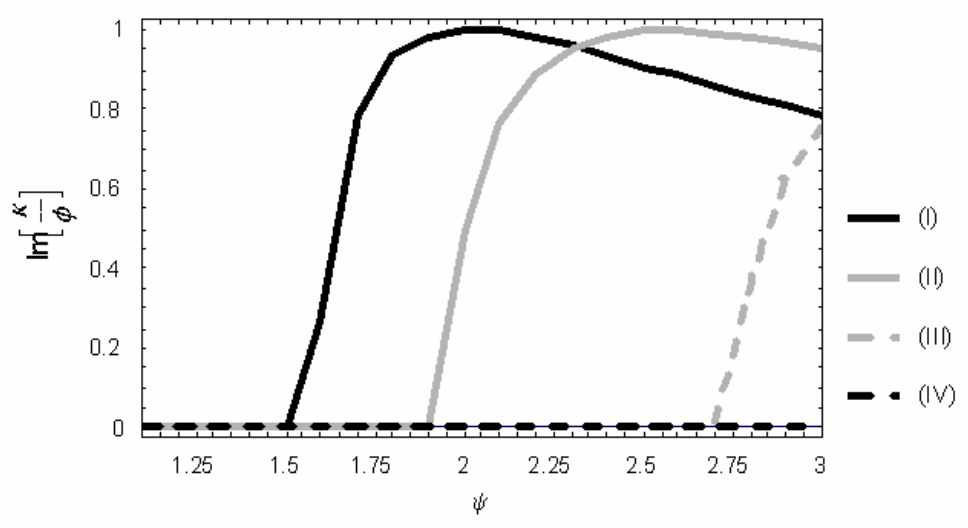

Gráfico 3.5 - Imagem no eixo de Gauss da relação $\kappa / \phi$ para o caso analisado.

Em tese, elementos finitos com reduzida ordem de erro na aproximação numérica da freqüência espacial sugerem apresentar melhor comportamento nas operações da análise modal.

Seja então agora o caso de se encontrar as freqüências naturais da barra simplesmente engastada mostrada na figura 2.7. Conforme a integração espacial proposta, as matrizes de massa e de rigidez do elemento podem ser redigidas na forma genérica (3.23):

$$
[\mathrm{M}]=\left[\begin{array}{cc}
\frac{1}{2}-\beta_{\mathrm{s}} & \beta_{\mathrm{s}} \\
\beta_{\mathrm{s}} & \frac{1}{2}-\beta_{\mathrm{s}}
\end{array}\right] \quad ; \quad[\mathrm{K}]=\mathrm{n}^{2} \mathrm{f}_{\mathrm{R}}^{2}\left[\begin{array}{cc}
1 & -1 \\
-1 & 1
\end{array}\right]
$$

com:

$$
f_{R}=\frac{C_{R}}{L_{T}}
$$


onde a razão $C_{R} / L_{T}$ estabelece a freqüência da onda percorrendo a barra em questão, aqui com comprimento $L_{T}$. Resolvendo-se de forma analítica, a freqüência natural nesse exemplo é resulta:

$$
\omega_{\mathrm{k}}=\frac{\mathrm{C}_{\mathrm{R}}}{\mathrm{L}_{\mathrm{T}}}\left[\frac{\pi}{2}+(\mathrm{k}-1) \pi\right] \quad(\mathrm{k}=1,2, \ldots, \mathrm{n})
$$

onde k representa o modo de vibração.

Nesse caso tem-se $f_{R}=400 \mathrm{~Hz}$, e as tabelas $3.1,3.2,3.3$ e 3.4 arrolam os resultados das freqüências naturais do sistema discretizado com o modelo clássico de massa consistente, o modelo de ordem elevada, o de massa concentrada e o correspondente incondicionalmente estável $(\beta=1 / 4)$ no espaço respectivamente.

\begin{tabular}{|c|c|c|c|c|c|c|}
\hline \multicolumn{7}{|c|}{$\beta \mathrm{s}=1 / 6$ (modelo consistente clássico) } \\
\hline \multirow{2}{*}{$\begin{array}{c}\text { Número de } \\
\text { elementos }\end{array}$} & \multicolumn{7}{|c|}{ Freqüências $(\mathrm{Hz})$} \\
\hline & $1^{\circ}$ modo & $2^{\circ}$ modo & $3^{\circ}$ modo & $4^{\circ}$ modo & $5^{\circ}$ modo & $6^{\circ}$ modo \\
\hline $\mathrm{n}=2$ & 102.6 & 358.4 & ------ & ------ & ------ & ------ \\
\hline $\mathrm{n}=3$ & 101.1 & 330.8 & 600.1 & ----- & ----- & ----- \\
\hline $\mathrm{n}=4$ & 100.6 & 317.5 & 576.7 & 834.0 & ----- & ------ \\
\hline $\mathrm{n}=5$ & 100.4 & 311.2 & 551.3 & 826.7 & 1063.4 & ------ \\
\hline $\mathrm{n}=10$ & 100.1 & 302.8 & 512.9 & 735.6 & 975.3 & 1235.1 \\
\hline $\mathrm{n}=15$ & 100.0 & 301.2 & 505.7 & 715.8 & 933.6 & 1161.3 \\
\hline Analítico & $\mathbf{1 0 0}$ & $\mathbf{3 0 0}$ & $\mathbf{5 0 0}$ & $\mathbf{7 0 0}$ & $\mathbf{9 0 0}$ & $\mathbf{1 1 0 0}$ \\
\hline
\end{tabular}

Tabela 3.1 - Freqüências naturais $(\mathrm{em} \mathrm{Hz})$ para $\beta_{\mathrm{s}}=1 / 6$.

\begin{tabular}{|c|c|c|c|c|c|c|}
\hline \multicolumn{7}{|c|}{$\beta \mathrm{s}=1 / 12$ (modelo de ordem elevada) } \\
\hline $\begin{array}{c}\text { Número de } \\
\text { elementos }\end{array}$ & $1^{\circ}$ modo & $2^{\circ}$ modo & $3^{\circ}$ modo & $4^{\circ}$ modo & $5^{\circ}$ modo & $6^{\circ}$ modo \\
\hline $\mathrm{n}=2$ & 99.9 & 278.1 & ------ & ------ & ------ & ------- \\
\hline $\mathrm{n}=3$ & 100.0 & 295.9 & 444.5 & ------- & ------ & ------- \\
\hline $\mathrm{n}=4$ & 100.0 & 298.7 & 482.7 & 606.0 & ------ & ------- \\
\hline $\mathrm{n}=5$ & 100.0 & 299.5 & 493.1 & 661.5 & 765.4 & ------ \\
\hline $\mathrm{n}=10$ & 100.0 & 300.0 & 499.6 & 697.8 & 892.0 & 1077.6 \\
\hline $\mathrm{n}=15$ & 100.0 & 300.0 & 499.9 & 699.6 & 898.5 & 1095.8 \\
\hline Analítico & $\mathbf{1 0 0}$ & $\mathbf{3 0 0}$ & $\mathbf{5 0 0}$ & $\mathbf{7 0 0}$ & $\mathbf{9 0 0}$ & $\mathbf{1 1 0 0}$ \\
\hline
\end{tabular}

Tabela 3.2 - Freqüências naturais $\left(\mathrm{em} \mathrm{Hz}\right.$ ) para $\beta_{\mathrm{s}}=1 / 12$. 


\begin{tabular}{|c|c|c|c|c|c|c|}
\hline \multicolumn{7}{|c|}{$\beta \mathrm{s}=\mathbf{0}$ (modelo massa concentrada) } \\
\hline \multirow{2}{*}{$\begin{array}{c}\text { Número de } \\
\text { elementos }\end{array}$} & \multicolumn{7}{|c|}{ Freqüências $(\mathrm{Hz})$} \\
\hline & $1^{\circ}$ modo & $2^{\circ}$ modo & $3^{\circ}$ modo & $4^{\circ}$ modo & $5^{\circ}$ modo & $6^{\circ}$ modo \\
\hline $\mathrm{n}=2$ & 97.4 & 235.3 & ----- & ----- & ----- & ----- \\
\hline $\mathrm{n}=3$ & 98.9 & 270.1 & 369.0 & ----- & ----- & ----- \\
\hline $\mathrm{n}=4$ & 99.4 & 283.0 & 423.5 & 499.5 & ----- & ----- \\
\hline $\mathrm{n}=5$ & 99.6 & 289.0 & 450.2 & 567.2 & 628.8 & ----- \\
\hline $\mathrm{n}=10$ & 99.9 & 297.2 & 487.2 & 665.3 & 826.9 & 968.2 \\
\hline $\mathrm{n}=15$ & 100.0 & 298.8 & 494.3 & 684.4 & 867.1 & 1040.2 \\
\hline Analítico & $\mathbf{1 0 0}$ & $\mathbf{3 0 0}$ & $\mathbf{5 0 0}$ & $\mathbf{7 0 0}$ & $\mathbf{9 0 0}$ & $\mathbf{1 1 0 0}$ \\
\hline
\end{tabular}

Tabela 3.3 - Freqüências naturais $(\mathrm{em} \mathrm{Hz})$ para $\beta_{\mathrm{s}}=0$.

\begin{tabular}{|c|c|c|c|c|c|c|}
\hline \multicolumn{7}{|c|}{$\beta \mathrm{s}=1 / 4$ (modelo incondicionalmente estável) } \\
\hline \multirow{2}{*}{$\begin{array}{c}\text { Número de } \\
\text { elementos }\end{array}$} & \multicolumn{7}{|c|}{ Freqüências (Hz) } \\
\cline { 2 - 8 } & $1^{\circ}$ modo & $2^{\circ}$ modo & $3^{\circ}$ modo & $4^{\circ}$ modo & $5^{\circ}$ modo & $6^{\circ}$ modo \\
\hline $\mathrm{n}=2$ & 105.5 & 614.8 & ----- & ------ & ------ & ------ \\
\hline $\mathrm{n}=3$ & 102.3 & 382.0 & 1425.5 & ----- & ----- & ------ \\
\hline $\mathrm{n}=4$ & 101.3 & 340.3 & 762.2 & 2560.4 & ----- & ----- \\
\hline $\mathrm{n}=5$ & 100.8 & 324.4 & 636.6 & 1249.4 & 4019.5 & ------ \\
\hline $\mathrm{n}=10$ & 100.2 & 305.7 & 527.4 & 780.2 & 1087.5 & 1490.8 \\
\hline $\mathrm{n}=15$ & 100.1 & 302.5 & 511.7 & 733.1 & 973.1 & 1240.3 \\
\hline Analítico & $\mathbf{1 0 0}$ & $\mathbf{3 0 0}$ & $\mathbf{5 0 0}$ & $\mathbf{7 0 0}$ & $\mathbf{9 0 0}$ & $\mathbf{1 1 0 0}$ \\
\hline
\end{tabular}

Tabela 3.4 - Freqüências naturais $(\mathrm{em} \mathrm{Hz})$ para $\beta_{\mathrm{s}}=1 / 4$.

De acordo com os resultados, nota-se que o elemento simples de barra modelado através da maximização da ordem de erro apresenta convergência extremamente acelerada se comparado com os outros modelos, demonstrando leve alongamento do período teórico para altas freqüências. 


\section{Capítulo 4}

\section{Propagação numérica em elementos simples de viga de Timoshenko}

\subsection{INTRODUČÃO}

Uma vez investigado o elemento de barra, o elemento de viga pode ser entendido como sendo uma extensão desse caso. Têm-se agora duas equações de equilíbrio de ações: uma de força no sentido transversal, e a outra de momento. Tais equações de equilíbrio resultam novamente em um problema de determinação de autovalores e autovetores.

Escolhe-se o elemento de viga clássico com dois nós e dois graus de liberdade em cada nó, desenvolvido segundo a teoria de viga de Timoshenko. Inicia-se, como no capítulo anterior, apresentando-se a solução analítica da teoria 
de viga de Timoshenko, com o intuito de se confrontar com a solução numérica, que é desenvolvida logo em seguida.

A novidade que surge no caso da propagação das ondas de flexão reside no fato de que tais ondas apresentam natureza dispersiva, ou seja, a velocidade de propagação depende da freqüência. Por outro lado, a simulação numérica introduz dispersão adicional, além de contemplar bifurcação resultando-se agora em ondas evanascentes.

\subsection{EQUACÃA DE MOVIMENTO}

Segundo a teoria clássica de flexão de Timoshenko, um elemento de viga infinitesimal em vibração tem a geometria deformada e ações atuantes conforme ilustra-se na figura 4.1. A reta tracejada é tangente ao eixo central da viga, enquanto a linha pontilhada é normal à seção transversal. A força cortante e o momento fletor estão representados segundo a notação $\vee$ e $M$, respectivamente.

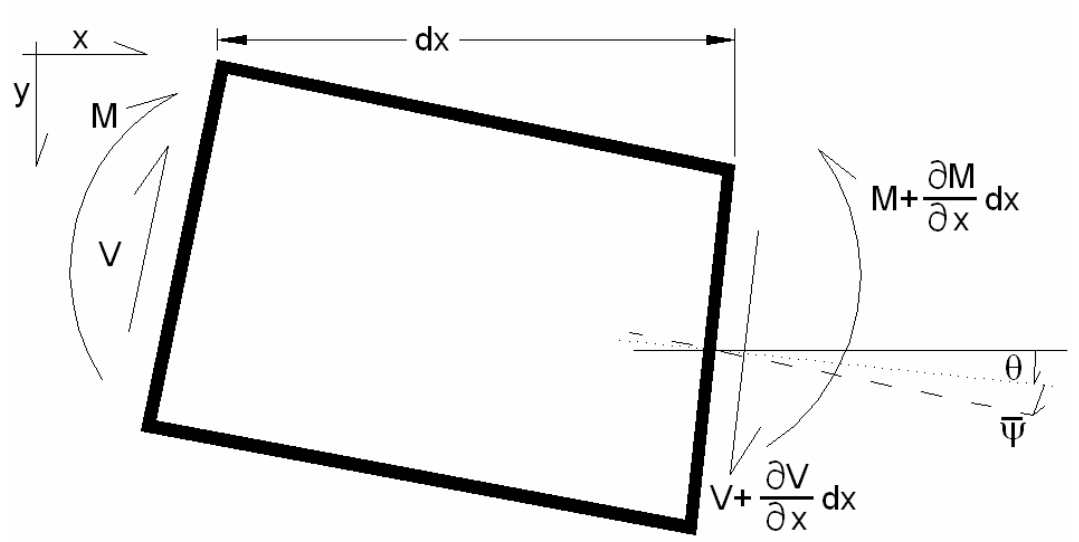

Figura 4.1 - Ações e geometria em um elemento infinitesimal de viga.

A elástica da viga, denotada pela variável y, tem como componentes de sua derivada primeira a rotação $\theta$, devida ao momento fletor, e a rotação $\bar{\psi}$ devida à distorção por cisalhamento (esforço cortante). Portanto:

$$
\frac{\partial y}{\partial x}=\theta+\bar{\psi}
$$


O equilíbrio de forças verticais neste caso é composto de duas parcelas: a primeira é a força de inércia de D’Alembert transversal, e a segunda é dada pela variação (incremento) de força cortante, que resulta da multiplicação da distorção $\bar{\psi}$ pela rigidez $\bar{\phi}=\overline{\mathrm{k}} \mathrm{G}$, onde $\mathrm{G}$ é o módulo de elasticidade transversal, A a área da seção transversal, e $\bar{k}$ o coeficiente de cisalhamento que depende da seção transversal e do coeficiente de Poisson v [COWPER (1966)]. Assim, a primeira equação diferencial de movimento é dada por [FRANKLIN (1970)]:

$$
\rho A \frac{\partial^{2} y}{\partial t^{2}}-\bar{\phi}\left(\frac{\partial^{2} y}{\partial x^{2}}-\frac{\partial \theta}{\partial x}\right)=0
$$

No equilíbrio de momentos acham-se envolvidas a contribuição do momento oriundo da inércia de rotação, de acordo com o princípio de D’Alembert, a contribuição do momento fletor atuante na viga, que relaciona-se constitutivamente com a componente de giro $\theta$, e, finalmente, a contribuição da força mediante seu binário elementar. Dessa forma a segunda equação de movimento de momentos se exprime:

$$
\rho \mathrm{l} \frac{\partial^{2} \theta}{\partial \mathrm{t}^{2}}-\mathrm{El} \frac{\partial^{2} \theta}{\partial \mathrm{x}^{2}}-\bar{\phi}\left(\frac{\partial \mathrm{y}}{\partial \mathrm{x}}-\theta\right)=0
$$

onde I é o momento de área da seção transversal.

Por outro lado, dividindo (4.2) e (4.3) por $\rho$ A, tem-se [BILLGER (1998)]:

$$
\begin{aligned}
& \ddot{y}-c_{S}^{2}\left(y^{\prime \prime}-\theta^{\prime}\right)=0 \\
& r^{2} \ddot{\theta}-r^{2} c_{R}^{2} \theta^{\prime \prime}-c_{S}^{2}\left(y^{\prime}-\theta\right)=0
\end{aligned}
$$

onde $r$ e cs são, respectivamente, o raio de giração e a velocidade de cisalhamento de referência, que se expressam:

$$
r=\sqrt{\frac{I}{A}} \quad ; \quad c_{s}=\sqrt{\frac{\bar{k} G}{A}}
$$


valendo-se notar que $c_{s}$ pode ser entendida como velocidade de uma onda de distorção, caso fosse possível não haver deformação por flexão.

A solução não finita (movimento ondulatório) das equações diferenciais parciais (4.4) pode ser expressa em notação complexa como:

$$
y(x, t)=C_{1} e^{i(\alpha x+\omega t)} \quad ; \quad \theta(x, t)=C_{1}^{*} e^{i(\alpha x+\omega t)}
$$

onde $\mathrm{C}_{\mathrm{i}}$ são as amplitudes dos movimentos acoplados da onda em questão.

A substituição destas em (4.4), após algumas operações algébricas, retorna o sistema:

$$
\begin{aligned}
& -\omega^{2} C_{1}+c_{S}^{2}\left(\alpha^{2} C_{1}+i \alpha C_{1}^{*}\right)=0 \\
& r^{2} \omega^{2} C_{1}^{*}-r^{2} C_{R}^{2} \alpha^{2} C_{1}^{*}+c_{S}^{2}\left(i \alpha C_{1}-C_{1}^{*}\right)=0
\end{aligned}
$$

que, em notação matricial, ganha a seguinte redação:

$$
\left[\begin{array}{cc}
\alpha^{2} c_{S}^{2}-\omega^{2} & i \alpha c_{S}^{2} \\
i \alpha c_{S}^{2} & r^{2} \omega^{2}-r^{2} c_{R}^{2} \alpha^{2}-c_{S}^{2}
\end{array}\right]\left\{\begin{array}{l}
C_{1} \\
C_{1}^{*}
\end{array}\right\}=\left\{\begin{array}{l}
0 \\
0
\end{array}\right\}
$$

resultando-se, pois, num problema de autovalor, que, para uma dada freqüência $\omega$, tem como auto valor a freqüência espacial $\alpha$.

A solução não trivial ocorre quando o determinante da matriz do primeiro membro se anula, ou seja:

$$
-r^{2} c_{R}^{2} c_{S}^{2} \alpha^{4}+\left(r^{2} \omega^{2} c_{R}^{2}+c_{S}^{2}+r^{2} \omega^{2} c_{S}^{2}\right) \alpha^{2}-r^{2} \omega^{4}=0
$$

cujas raízes em $\alpha$ são dadas por:

$$
\alpha= \pm \frac{\sqrt{2} \pi}{\lambda_{R}} \sqrt{\left(1+\gamma^{2}\right) \pm \sqrt{\left(1+\gamma^{2}\right)^{2}-4 \gamma^{2}\left[1-\left(\frac{\lambda_{R}}{2 \pi r \gamma}\right)^{2}\right]}}
$$

onde $\gamma$ é a razão $c_{R} / c_{S}$. Pode-se concluir que $\gamma$ depende apenas da geometria da seção transversal e do coeficiente de Poisson. A igualdade (4.10) indica que são 
dois os pares de ondas acopladas presentes no movimento. Portanto, os par de raízes positivas são:

$$
\begin{aligned}
& \alpha_{1}=\frac{\sqrt{2} \pi}{\lambda_{R}} \sqrt{\left(1+\gamma^{2}\right)-\sqrt{\left(1+\gamma^{2}\right)^{2}-4 \gamma^{2}\left[1-\left(\frac{\lambda_{R}}{2 \pi r \gamma}\right)^{2}\right]}} \\
& \alpha_{2}=\frac{\sqrt{2} \pi}{\lambda_{R}} \sqrt{\left(1+\gamma^{2}\right)+\sqrt{\left(1+\gamma^{2}\right)^{2}-4 \gamma^{2}\left[1-\left(\frac{\lambda_{R}}{2 \pi r \gamma}\right)^{2}\right]}}
\end{aligned}
$$

onde $\alpha_{1}$ e $\alpha_{2}$ são respectivamente a primeira e segunda freqüência espacial resultante. Os autovetores de (4.8) para a primeira e a segunda raizes são dados respectivamente por:

$$
\left\{\begin{array}{c}
1 \\
\Lambda_{1}
\end{array}\right\} \quad ; \quad\left\{\begin{array}{c}
1 \\
\Lambda_{2}
\end{array}\right\}
$$

com:

$$
\Lambda_{j}=i \frac{\alpha_{j}^{2} c_{S}^{2}-\omega^{2}}{\alpha_{j} c_{S}^{2}}=i \frac{\sqrt{2} \pi}{\lambda_{R}}\left(\frac{1-\gamma^{2} \mp\left(\sqrt{\left(1+\gamma^{2}\right)^{2}-4 \gamma^{2}\left[1-\left(\frac{\lambda_{R}}{2 \pi r \gamma}\right)^{2}\right]}\right.}{\sqrt{1+\gamma^{2} \mp \sqrt{\left(1+\gamma^{2}\right)^{2}-4 \gamma^{2}\left[1-\left(\frac{\lambda_{R}}{2 \pi r \gamma}\right)^{2}\right]}}}\right) \quad(j=1,2)
$$

onde considera-se unitária a primeira componente do autovetor.

É oportuno atentar-se para o fato de que dois diferentes casos de solução ocorrem, quais sejam, um com dois pares de raízes imaginárias e outro com um par de raízes imaginárias e um par de raízes reais. Conforme mencionado no capítulo anterior, o caso onde a freqüência espacial assume valor imaginário caracteriza o fenômeno de onda dita evanescente. Esta divisão de soluções está associada à existência de bifurcação nas condições do movimento. De acordo com (4.11a), a primeira freqüência espacial é imaginária quando:

$$
\left(1+\gamma^{2}\right)-\sqrt{\left(1+\gamma^{2}\right)^{2}-4 \gamma^{2}\left[1-\left(\frac{\lambda_{R}}{2 \pi r \gamma}\right)^{2}\right]}<0
$$


Sendo evidente que esta inequação é satisfeita quando:

$$
\lambda_{R}>2 \pi r \gamma
$$

e cuja implicação no comportamento ondulatório será discutida em detalhe oportunamente.

\subsection{CÁLCULO DA PROPAGACÃO NUMÉRICA}

O elemento finito simples de viga modelado segundo a teoria de Timoshenko contém dois graus de liberdade em cada extremidade: um de translação e outro de rotação. A figura 4.2 ilustra os sentidos das coordenadas.

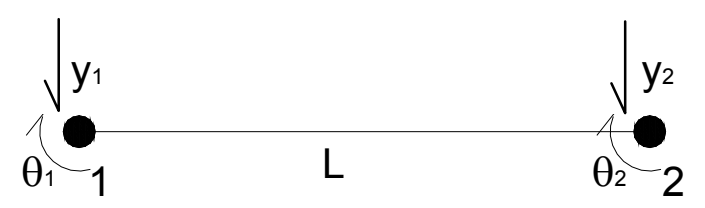

Figura 4.2 - Elemento simples de viga de Timoshenko.

As matrizes de massa e de rigidez obtidas pela formulação clássica, bem como seus parâmetros, são apresentadas no que se segue [THOMAS (1973)]:

$$
\begin{aligned}
& {[M]=\frac{\rho A L}{(1+\Phi)^{2}}\left(\left[\begin{array}{cccc}
m_{1} & m_{2} & m_{3} & m_{4} \\
& m_{5} & -m_{4} & m_{6} \\
& & m_{1} & -m_{2} \\
S I M & & & m_{5}
\end{array}\right]+\frac{1}{A^{2}}\left[\begin{array}{cccc}
m_{7} & m_{8} & -m_{7} & m_{8} \\
& m_{9} & -m_{8} & m_{10} \\
& m_{7} & -m_{8} \\
S I M & & m_{9}
\end{array}\right]\right)} \\
& {[K]=\frac{E I}{L^{3}(1+\Phi)}\left[\begin{array}{cccc}
12 & 6 L & -12 & 6 L \\
& L^{2}(4+\Phi) & -6 L & L^{2}(2-\Phi) \\
S I M & 12 & -6 L \\
& & L^{2}(4+\Phi)
\end{array}\right]} \\
& \Phi=\frac{12 E I}{L^{2} \phi}
\end{aligned}
$$




$$
\begin{array}{lll}
\mathrm{m}_{1}=\frac{13}{35}+\frac{7 \Phi}{10}+\frac{\Phi^{2}}{3} & ; & \mathrm{m}_{2}=\left(\frac{11}{210}+\frac{11 \Phi}{120}+\frac{\Phi^{2}}{24}\right) \mathrm{L} \\
\mathrm{m}_{3}=\frac{9}{70}+\frac{3 \Phi}{10}+\frac{\Phi^{2}}{6} & ; & \mathrm{m}_{4}=-\left(\frac{13}{420}+\frac{3 \Phi}{40}+\frac{\Phi^{2}}{24}\right) \mathrm{L} \\
\mathrm{m}_{5}=\left(\frac{1}{105}+\frac{\Phi}{60}+\frac{\Phi^{2}}{120}\right) \mathrm{L}^{2} ; & \mathrm{m}_{6}=-\left(\frac{1}{140}+\frac{\Phi}{60}+\frac{\Phi^{2}}{120}\right) \mathrm{L}^{2} \\
\mathrm{~m}_{7}=\frac{6}{5} & \mathrm{~m}_{8}=\left(\frac{1}{10}-\frac{\Phi}{2}\right) \mathrm{L} \\
\mathrm{m}_{9}=\left(\frac{2}{15}+\frac{\Phi}{6}+\frac{\Phi^{2}}{3}\right) \mathrm{L}^{2} & ; & \mathrm{m}_{10}=\left(-\frac{1}{30}-\frac{\Phi}{6}+\frac{\Phi^{2}}{6}\right) \mathrm{L}^{2}
\end{array}
$$

que, como sabido, trata-se de um eficiente elemento finito desprovido do travamento de cortante

Para o estudo da propagação ondulatória pela via numérica, considere-se uma viga de comprimento infinito, dividida em elementos finitos uniformes, sendo percorrida por uma onda de flexão, propagando-se da esquerda para a direita, como ilustrado na figura 4.3, na qual se detalha apenas o entorno de um nó genérico j.

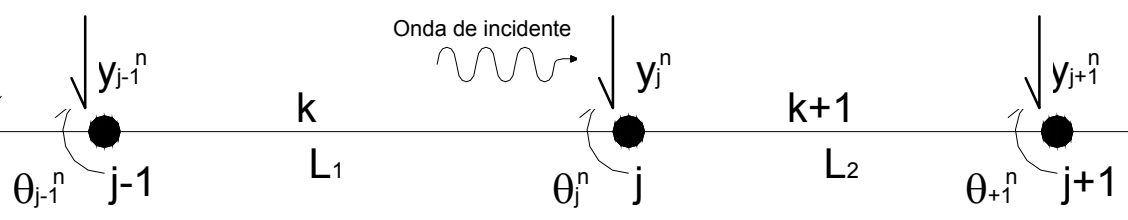

Figura 4.3 - Viga de comprimento infinito sujeita a uma onda de incidente.

O equilíbrio de força transversal e momento referente ao nó genérico j, em redação matricial, ganha a seguinte escrita:

$$
\left[\mathrm{K}_{\mathrm{k}}\right]\{\mathrm{u}\}_{\mathrm{j}}^{\mathrm{n}}+\left[\mathrm{K}_{\mathrm{k}+1}\right]\{\mathrm{u}\}_{\mathrm{j}+1}^{\mathrm{n}}+\left[\mathrm{M}_{\mathrm{k}}\right]\{\ddot{\mathrm{u}}\}_{\mathrm{j}}^{\mathrm{n}}+\left[\mathrm{M}_{\mathrm{k}+1}\right]\{\ddot{\mathrm{u}}\}_{\mathrm{j}+1}^{\mathrm{n}}=\{0\}
$$

com: 


$$
\begin{gathered}
{\left[M_{k}\right]=\left[\begin{array}{cccc}
m_{3} & -m_{4} & m_{1} & -m_{2} \\
m_{4} & m_{6} & -m_{2} & m_{5}
\end{array}\right]+\delta^{2}\left[\begin{array}{cccc}
-m_{7} & -m_{8} & m_{7} & -m_{8} \\
m_{8} & m_{10} & -m_{8} & m_{9}
\end{array}\right]} \\
{\left[M_{k+1}\right]=\left[\begin{array}{cccc}
m_{1} & m_{2} & m_{3} & m_{4} \\
m_{2} & m_{5} & -m_{4} & m_{6}
\end{array}\right]+\delta^{2}\left[\begin{array}{cccc}
m_{7} & m_{8} & -m_{7} & m_{8} \\
m_{8} & m_{9} & -m_{8} & m_{10}
\end{array}\right]} \\
{\left[K_{k}\right]=\frac{\left(1+12 \gamma^{2} \delta^{2}\right) p^{2} \delta^{2} \xi^{2}}{T^{2}}\left[\begin{array}{cccc}
-12 & -6 L & 12 & -6 L \\
6 L & \left(2-12 \gamma^{2} \delta^{2}\right)^{2} & -6 L & \left(4+12 \gamma^{2} \delta^{2}\right)^{2}
\end{array}\right]} \\
{\left[K_{k+1}\right]=\frac{\left(1+12 \gamma^{2} \delta^{2}\right) p^{2} \delta^{2} \xi^{2}}{T^{2}}\left[\begin{array}{cccc}
12 & 6 L & -12 & 6 L \\
6 L & \left(4+12 \gamma^{2} \delta^{2}\right)^{2} & -6 L & \left(2-12 \gamma^{2} \delta^{2}\right)^{2}
\end{array}\right]} \\
\delta=\frac{r}{L} \quad\left\{\quad\{u\}_{j}^{n}=\left\{\begin{array}{llll}
y_{j-1}^{n} & \theta_{j-1}^{n} & y_{j}^{n} & \left.\theta_{j}^{n}\right\}^{\top}
\end{array}\right.\right.
\end{gathered}
$$

onde a notação $\left[\mathrm{M}_{\mathrm{k}}\right]$ e $\left[\mathrm{K}_{\mathrm{k}}\right]$ indicam as contribuições das matrizes de massa e de rigidez respectivamente do elemento $k$, valendo a mesma para a contribuição do elemento $k+1$; sendo que a primeira linha refere-se ao equilíbrio de forças transversais e a segunda ao equilíbrio de momentos.

Para caracterizar uma onda que se propaga da esquerda para a direita, considerando como origem do sistema o nó j, emprega-se um campo de deslocamento numérico na forma [LUNDÉN (1983)]:

$$
y_{j}^{n}=C \cdot e^{i\left(j \alpha_{N} L-n \omega T\right)} \quad ; \quad \theta_{j}^{n}=C^{*} e^{i\left(j \alpha_{N} L-n \omega T\right)}
$$

Implicando nos vetores de deslocamentos:

$$
\begin{aligned}
& \left\{u_{j}^{n}\right\}=\left\{u_{j}^{*}\right\} e^{-i n(2 \pi \xi)} \\
& \left\{u_{j}^{*}\right\}^{\top}=\left\{\begin{array}{llll}
C e^{i \alpha_{N} L} & C^{*} e^{i \alpha_{N} L} & C & C^{*}
\end{array}\right\} \\
& \left\{u_{j+1}^{*}\right\}^{\top}=\left\{\begin{array}{llll}
C & C^{*} & C e^{-i \alpha_{N} L} & C^{*} e^{-i \alpha_{N} L}
\end{array}\right\}
\end{aligned}
$$


Matematicamente, o fato de os elementos terem o mesmo comprimento $L$ torna os vetores $\left\{u_{j}^{*}\right\}^{\top}$ e $\left\{u_{j+1}^{*}\right\}^{\top}$ linearmente dependentes, podendo-se obter uma relação direta entre eles do tipo:

$$
\left\{u_{j}^{*}\right\}^{\top}=e^{i \alpha_{N} L}\left\{u_{j+1}^{*}\right\}^{\top}
$$

fato que permite uma nova redação para a equação (4.17), ou seja:

$$
[\bar{M}]\left\{\dot{u}_{j+1}^{n}\right\}+[\bar{K}]\left\{u_{j+1}^{n}\right\}=\{0\}
$$

com:

$$
[\bar{M}]=e^{i \alpha_{N} L}\left[M_{k}\right]+\left[M_{k+1}\right] \quad ; \quad[\bar{K}]=e^{i \alpha_{N} L}\left[K_{k}\right]+\left[K_{k+1}\right]
$$

onde já se considera uma notação mais compacta para as matrizes envolvidas.

Integrando-se a equação (4.22) no tempo com o emprego do integrador trapezoidal de Newmark (passo duplo) conforme a redação (2.12), tem-se:

$$
\nabla[\overline{\mathrm{M}}]\left\{\mathrm{u}_{\mathrm{j}+1}^{\mathrm{n}}\right\}+(1+\beta \nabla)\left[\overline{\mathrm{K}}^{*}\right]\left\{\mathrm{u}_{\mathrm{j}+1}^{\mathrm{n}}\right\}=\{0\}
$$

onde $\left[\overline{\mathrm{K}}^{*}\right]$ representa o produto $\mathrm{T}^{2}[\overline{\mathrm{K}}]$, com o operador $\nabla$ sendo definido em (2.13).

Recorrendo-se à relação (2.15), pode-se reescrever (4.24) na forma:

$$
\left\lfloor\left(\beta \cos (2 \pi \xi)-\beta+\frac{1}{2}\right) \mid \bar{K}^{*}\right\rfloor+(\cos (2 \pi \xi)-1)[\bar{M}] \mid\left\{u_{j+1}^{n}\right\}=\{0\}
$$

resultando-se, pois, na equação de diferenças regente da propagação ondulatória da versão numérica relativa a esse elemento finito clássico de viga de Timoshenko.

$\mathrm{Na}$ manipulação matemática da equação de diferenças, visando uma conveniente redação da equação de movimento, é oportuno o emprego das seguintes relações envolvendo as matrizes do elemento j e j+1:

$$
\left[\mathrm{K}_{\mathrm{k}}\right]=[\mathrm{A}]\left[\mathrm{K}_{\mathrm{k}+1}\right][\mathrm{B}] \quad ; \quad\left[\mathrm{M}_{\mathrm{k}}\right]=[\mathrm{A}]\left[\mathrm{M}_{\mathrm{k}+1}\right][\mathrm{B}]
$$

com: 


$$
[A]=\left[\begin{array}{cccc}
0 & 0 & 1 & 0 \\
0 & 0 & 0 & -1 \\
1 & 0 & 0 & 0 \\
0 & -1 & 0 & 0
\end{array}\right] \quad ; \quad[B]=\left[\begin{array}{cc}
1 & 0 \\
0 & -1
\end{array}\right]
$$

Assim sendo, examinando-se separadamente a matriz de rigidez da equação de diferenças (4.25), face ao exposto em (4.26), tem-se:

$$
\left.\left.\left[\bar{K}^{*}\right]\left\{u_{j+1}^{*}\right\}=[B]\left[K_{k+1}^{*}\right] A\right]\right]\left\{u_{j+1}^{*}\right\} e^{i a_{N} L}+\left[K_{k+1}^{*}\right]\left\{u_{j+1}^{*}\right\}
$$

onde:

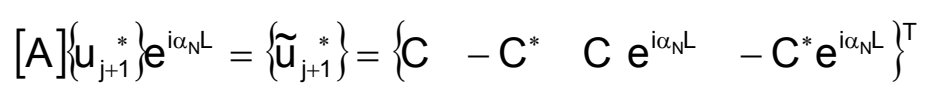

Além disso, é também oportuno proceder a seguinte decomposição da matriz [B] em duas outras, como mostrado no que se segue, o que confere uma nova redação para o exposto em (4.23) na forma:

$$
\begin{aligned}
& {\left[\bar{K}^{*}\right]\left\{\mathbf{u}_{j+1}^{*}\right\}=\left[\begin{array}{ll}
1 & 0 \\
0 & 0
\end{array}\right]\left[\mathrm{K}_{\mathrm{k}+1}^{*}\right]\left\{\mathrm{U}_{1}\right\}+\left[\begin{array}{ll}
0 & 0 \\
0 & 1
\end{array}\right]\left[\mathrm{K}_{\mathrm{k}+1}^{*}\right]\left\{\mathrm{U}_{2}\right\}} \\
& \left\{U_{1}\right\}=\left\{u_{j+1}^{*}\right\}+\left\{\tilde{u}_{j+1}^{*}\right\}=2\left\{\begin{array}{lll}
C & 0 & C \cos \left(\alpha_{N} L\right)
\end{array}-i C^{*} \operatorname{sen}\left(\alpha_{N} L\right)\right\}^{\top}
\end{aligned}
$$

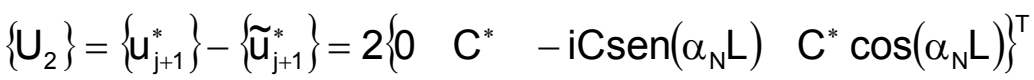

concluindo-se, assim, que é possível tratar o expresso em (4.28) já envolvendo apenas expressões trigonométricas, ou seja:

$$
\left[\bar{K}^{*}\right]\left[u_{j+1}^{*}\right\}=p^{2} \delta^{2} \xi^{2}\left[\begin{array}{cc}
12\left[\cos \left(\alpha_{N} L\right)-1\right] & i 6 \operatorname{Lsen}\left(\alpha_{N} L\right) \\
i 6 L \operatorname{sen}\left(\alpha_{N} L\right) & \left(4+12 \gamma^{2} \delta^{2}\right)+\left(2-12 \gamma^{2} \delta^{2}\right) \cos \left(\alpha_{N} L\right)
\end{array}\right]\left\{\begin{array}{c}
C \\
C^{*}
\end{array}\right\}
$$

Procedimento análogo pode ser realizado para o caso da matriz de massa em (4.25), implicando-se em: 
$[\bar{M}]\left\{u_{j+1}^{*}\right\}=\frac{1}{1+12 \gamma^{2} \delta^{2}}\left[\begin{array}{cc}\left(\delta^{2} m_{7}-m_{3}\right) \cos \left(\alpha_{N} L\right)-m_{1}-\delta^{2} m_{7} & i\left(m_{4}+\delta^{2} m_{8}\right) \operatorname{sen}\left(\alpha_{N} L\right) \\ i\left(m_{4}+\delta^{2} m_{8}\right) \operatorname{sen}\left(\alpha_{N} L\right) & m_{5}+\delta^{2} m_{9}+\left(m_{6}+\delta^{2} m_{10}\right) \operatorname{sen}\left(\alpha_{N} L\right)\end{array}\right]\left\{\begin{array}{c}C \\ C^{*}\end{array}\right\}$

resultando, em conseqüência de (4.31) e (4.32), que a equação (4.25) fica agora expressa como:

$$
\left[\begin{array}{cc}
D_{2} \cos \left(\alpha_{N} L\right)-D_{1} & i D_{3} \operatorname{sen}\left(\alpha_{N} L\right) \\
i D_{3} \operatorname{sen}\left(\alpha_{N} L\right) & D_{4}+D_{5} \cos \left(\alpha_{N} L\right)
\end{array}\right]\left\{\begin{array}{l}
C \\
C^{*}
\end{array}\right\}=\left\{\begin{array}{l}
0 \\
0
\end{array}\right\}
$$

onde:

$$
\begin{aligned}
& \mathrm{D}_{1}=12 \varphi_{\mathrm{K}}+\varphi_{\mathrm{M}}\left(\mathrm{m}_{1}+\delta^{2} \mathrm{~m}_{7}\right) ; \mathrm{D}_{2}=12 \varphi_{\mathrm{K}}-\varphi_{\mathrm{M}}\left(\mathrm{m}_{3}-\delta^{2} \mathrm{~m}_{7}\right) \\
& \mathrm{D}_{3}=6 \mathrm{~L} \varphi_{\mathrm{K}}+\varphi_{\mathrm{M}}\left(\mathrm{m}_{4}+\delta^{2} \mathrm{~m}_{8}\right) ; \mathrm{D}_{4}=\left(4+12 \gamma^{2} \delta^{2}\right) \varphi_{\mathrm{K}}+\varphi_{\mathrm{M}}\left(\mathrm{m}_{5}+\delta^{2} \mathrm{~m}_{9}\right) \\
& \mathrm{D}_{5}=\left(2-12 \gamma^{2} \delta^{2}\right) \varphi_{\mathrm{K}}+\varphi_{\mathrm{M}}\left(\mathrm{m}_{6}+\delta^{2} \mathrm{~m}_{10}\right) \\
& \varphi_{\mathrm{K}}=\mathrm{p}^{2} \delta^{2} \xi^{2}\left(\beta \cos (2 \pi \xi)-\beta+\frac{1}{2}\right) ; \varphi_{\mathrm{M}}=\frac{1}{1+12 \gamma^{2} \delta^{2}}(\cos (2 \pi \xi)-1)
\end{aligned}
$$

É interessante salientar que a expressão (4.8) é similar à (4.33a), uma vez que a expansão em série de Taylor das funções trigonométricas envolvidas resulta em uma aproximação daquela. A solução não trivial do sistema (4.33) é obtida anulando-se o determinante da matriz [D], implicando-se em:

$$
\cos ^{2}\left(\alpha_{N} L\right)-\sigma_{2} \cos \left(\alpha_{N} L\right)+\sigma_{1}=0
$$

com:

$$
\sigma_{1}=\frac{D_{3}^{2}-D_{1} D_{4}}{D_{2} D_{5}-D_{3}^{2}} \quad ; \quad \sigma_{2}=\frac{D_{1} D_{5}-D_{2} D_{4}}{D_{2} D_{5}-D_{3}^{2}}
$$

A expressão (4.35) é a equação característica disposta na forma de um polinômio do segundo grau em relação à variável $\cos \left(\alpha_{N} L\right)$. Observa-se que, para o caso da barra simples, de acordo com a expressão (2.20), a equação característica também se apresenta como um polinômio, porém de primeiro grau.

A solução da equação (4.35) em $\cos \left(\alpha_{N} L\right)$ é dada por: 


$$
\cos \left(\alpha_{N_{, 2},} L\right)=\frac{\sigma_{2} \pm \sqrt{\sigma_{2}^{2}-4 \sigma_{1}}}{2}
$$

onde $\alpha_{N, 1}$ e $\alpha_{N, 2}$ são, respectivamente, a primeira e a segunda freqüência espacial numérico para uma dada freqüência de onda $\omega$.

Para se encontrar a relação entre o valor numérico da freqüência espacial e o valor teórico da propagação, aqui denominado novamente pela letra grega $\eta$, divide-se $\alpha_{N}$ pertencente à equação (4.37) pela (4.11), resultando:

$$
\eta_{1}=\frac{\alpha_{N_{2}, 2}}{\alpha_{1}}=p \frac{\operatorname{arc.cos}\left(\frac{\sigma_{2} \pm \sqrt{\sigma_{2}^{2}-4 \sigma_{1}}}{2}\right)}{\sqrt{2} \pi \sqrt{\left(1+\gamma^{2}\right) \mp \sqrt{\left(1+\gamma^{2}\right)^{2}-4 \gamma^{2}\left[1-\left(\frac{p}{2 \pi \gamma \delta}\right)^{2}\right]}}}
$$

Como na modelagem do elemento foi admitida seção transversal constante, p e $\delta$ se relacionam linearmente, pois ambos são inversamente proporcionais ao comprimento do elemento. Esta constante de proporcionalidade pode ser obtida fazendo:

$$
Z=\frac{r}{\lambda_{R}}
$$

permitido-se que se escreva:

$$
\delta=Z p
$$

Respeitando a inequação (4.15), a primeira freqüência espacial é imaginária quando $Z$ é menor que $(2 \pi \gamma)^{-1}$. Devido à formulação adimensional empregada, pode-se concluir que o problema numérico necessita apenas de dois parâmetros físicos: $\gamma$ e Z. Admitindo os valores $\gamma=1.4, Z=1$ e $\beta=1 / 4$, a tabela 4.1 e a figura 4.4 reúnem valores de $\eta$ da primeira e da segunda freqüências espaciais para alguns casos de discretização. 


\begin{tabular}{|c|c|c|c|}
\hline \multicolumn{4}{|c|}{ Valores de $\eta_{1}$} \\
\hline & $\xi=0.2$ & $\xi=0.1$ & $\xi=0.05$ \\
\hline$p=5$ & 1.07487 & 0.97047 & 0.94781 \\
\hline$p=10$ & 1.13607 & 1.01694 & 0.9914 \\
\hline$p=20$ & 1.1548 & 1.03064 & 1.00415 \\
\hline
\end{tabular}

\begin{tabular}{|c|c|c|c|}
\hline \multicolumn{4}{|c|}{ Valores de $\eta_{2}$} \\
\hline & $\xi=0.2$ & $\xi=0.1$ & $\xi=0.05$ \\
\hline$p=5$ & 1.03491 & 0.94364 & 0.92381 \\
\hline$p=10$ & 1.11545 & 1.00597 & 0.98248 \\
\hline$p=20$ & 1.14327 & 1.02628 & 1.00137 \\
\hline
\end{tabular}

Tabela 4.1 - Valores de $\eta$ da primeira e da segunda freqüência espacial para alguns casos de integração espacial e temporal.

Nota-se claramente na figura (4.4), que o formato da curva é ditado pelo integrador temporal e que o ponto de cruzamento com a ordenada depende da discretização espacial.

a)

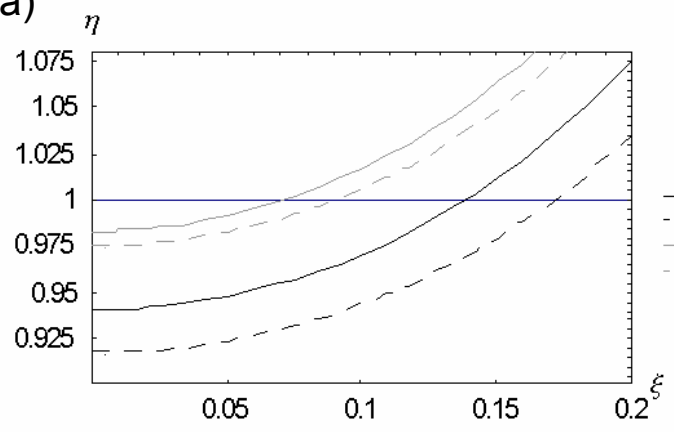

b)

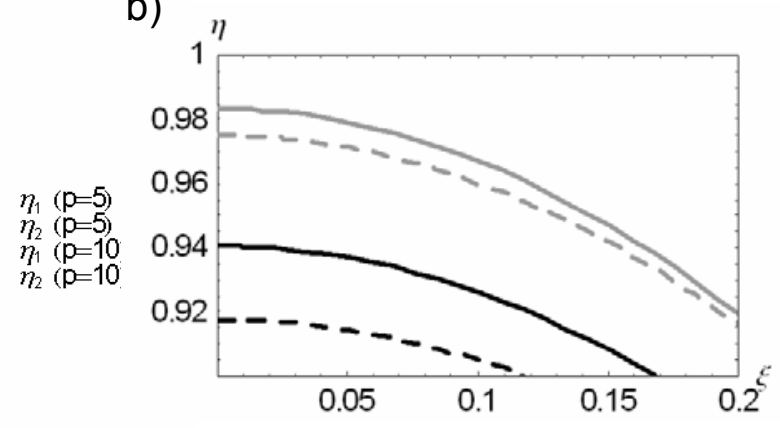

Figura 4.4 - Curva $\eta$ da primeira e da segunda freqüência espacial para casos com a) $\beta=1 / 4$ e b) $\beta=0$.

Assim como no caso uniaxial, o surgimento de ondas evanescentes acaba ocorrendo nos casos em que a freqüência espacial numérica resulta imaginária. $A$ superfície da figura 4.5 ilustra a imagem no eixo de Gauss de $\eta$ com $\gamma=1.4, p_{c}=5$, $\delta_{c}=5$ e $\beta=1 / 4$. Já a tabela 4.2 indica os valores de $p_{\text {Lim }}$ para tais casos.

a)

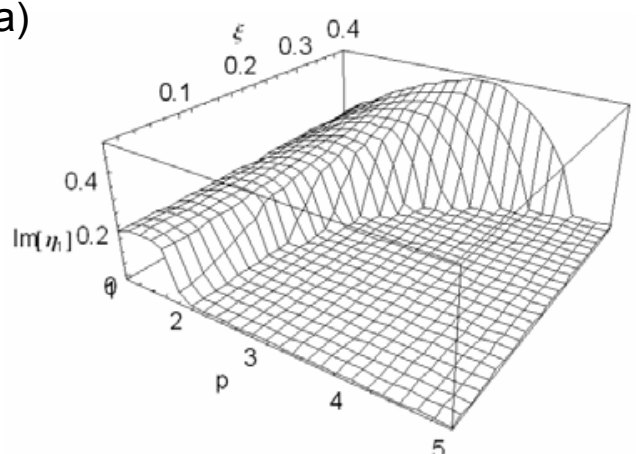

b)

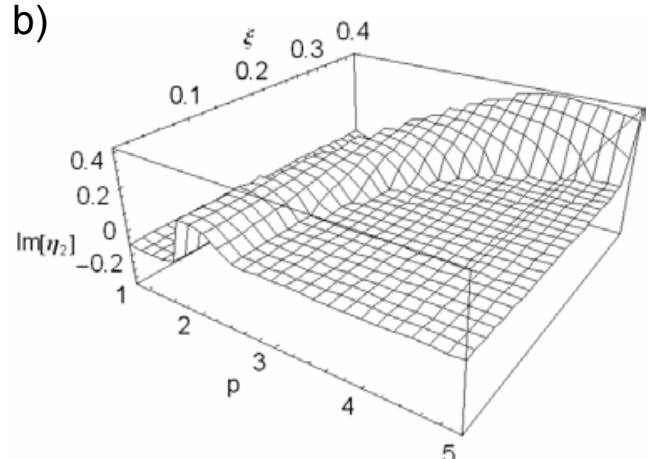

Figura 4.5 - Superfície de $\eta$ da primeira e da segunda freqüência espacial. 


\begin{tabular}{|c|c|c|c|}
\hline \multicolumn{4}{|c|}{ Valores de $\mathrm{p}_{\mathrm{Lim}}$} \\
\hline & $\xi=0.2$ & $\xi=0.1$ & $\xi=0.05$ \\
\hline $1^{\text {a }}$ freqüência espacial & 4.10406 & 3.65156 & 3.55513 \\
\hline $2^{\mathrm{a}}$ freqüência espacial & 5.51931 & 4.95034 & 4.82958 \\
\hline
\end{tabular}

Tabela 4.2 - Valores limites de p para o qual a onda se torna evanescente.

Visando-se verificar o comportamento da propagação ondulatória das ondas de Timoshenko, seja novamente considerado o caso ilustrado na figura 2.16, agora formado por elementos de viga, e com a modificação apenas do sentido de aplicação da força $F(t)=\operatorname{sen}(\omega t)$ (em kN), que passa a ser transversal; sendo que o módulo de elasticidade vale $E=2 \mathrm{kPa}$, a massa específica vale $\rho=2500 \mathrm{~kg} / \mathrm{m}^{3}$, o momento de área da seção transversal vale $\mathrm{I}=0.005 \mathrm{~m}^{4}$, a área da seção transversal vale $A=0.05 \mathrm{~m}^{2}$, o coeficiente de Poisson assume o valor $v=0.2$, o coeficiente de deformação por cortante da seção valendo $k=2 / 3$, o comprimento da viga em balanço vale $L_{T}=10 m$, o número de elementos $n=20$, o passo de tempo $\mathrm{T}=0.001 \mathrm{~s}$ e, finalmente, considera-se o caso do integrador com $\beta=1 / 4$.

Assim sendo, tem-se inicialmente que $\gamma=1.897, \delta=1.021$, e que a freqüência limite, para a qual ambas freqüências espaciais são evanescentes, passa a valer $16.0 \mathrm{~Hz}$.

No sentido de se visualizar melhor o tais movimento evanescentes, aplicase um filtro digital centrado nesta freqüência e outro que coleta a banda imediatamente após $16 \mathrm{~Hz}$, cujos sinais são respectivamente os fornecidos pelas linhas verde e azul da figura 4.6.

Cada uma das figuras 4.7 compara o deslocamento transversal do nó 1 (azul) com o do nó 20 (verde), ou seja, do primeiro com o último nó livre da viga. No primeiro, filtra-se a banda $10-25 \mathrm{~Hz}$ (verde) para uma excitação de $16 \mathrm{~Hz}$ e, no segundo, a banda 16-35 (azul) para uma excitação de 23Hz. Praticamente não há propagação de onda nos casos de movimento evanescente. 


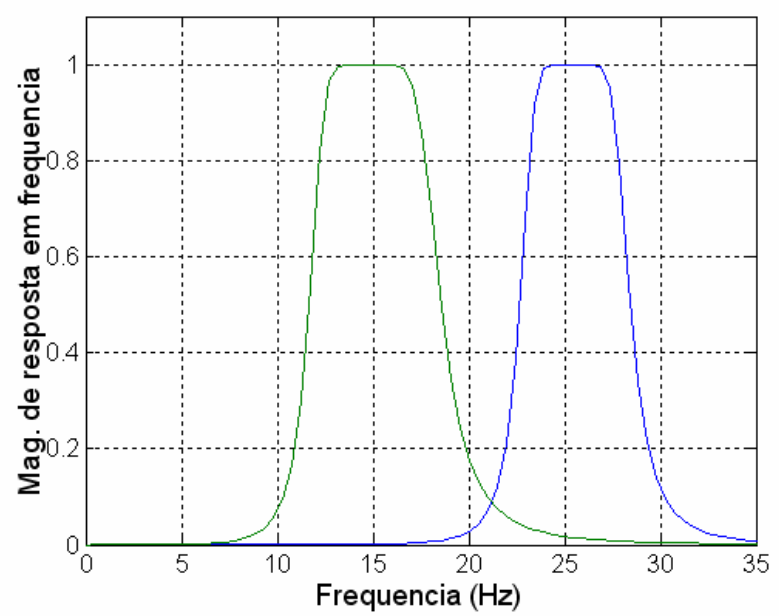

Figura 4.6 - Filtros empregados.

a)

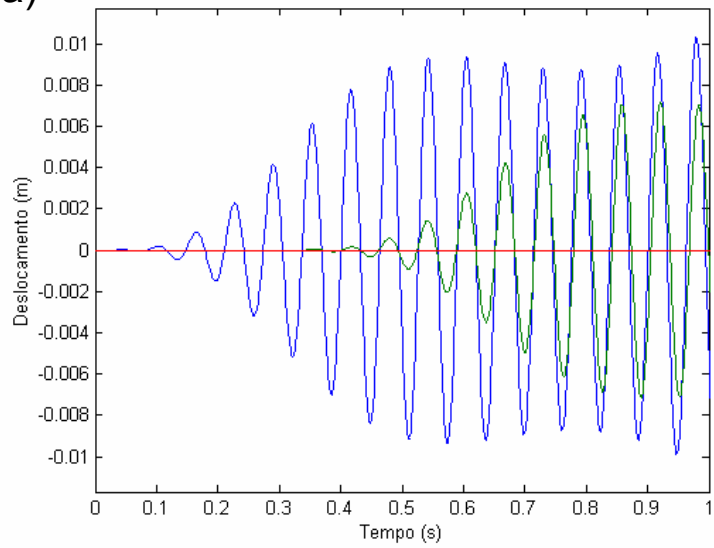

b)

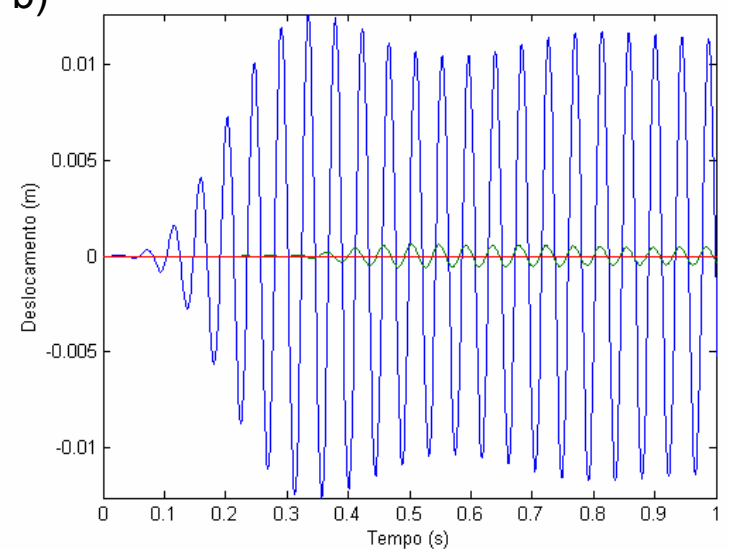

Figura 4.7 - Deslocamento transversal do nó 1 e do nó 20 (azul e verde respectivamente) para a) filtro $10-25 \mathrm{~Hz}$ e freqüência de excitação de $16 \mathrm{~Hz}$ e b) filtro $16-35 \mathrm{~Hz}$ com excitação de $23 \mathrm{~Hz}$.

A equação matricial (4.33a) tem como autovetores:

Primeira freqüência espacial: $\left\{\begin{array}{c}1 \\ \Lambda_{\mathrm{N}, 1}\end{array}\right\} \quad ;$ Segunda freqüência espacial: $\left\{\begin{array}{c}1 \\ \Lambda_{\mathrm{N}, 2}\end{array}\right\}$

e como $D_{1}$ e $D_{2}$ são adimensionais e $D_{3}$ é proporcional a $L$, tem-se:

$$
\Lambda_{\mathrm{N}, 2}=\frac{i}{L} \frac{D_{1}-D_{2} \cos \left(\alpha_{N_{2}} L\right)}{D_{3}^{*} \operatorname{sen}\left(\alpha_{N_{2}^{1}} L\right)}
$$

onde $D_{3}^{*}$ é a adimensionalização $D_{3} / L$. 
Finalmente, tendo-se em vista a expressão (4.13), a relação $\chi=\Lambda_{N} / \Lambda$ adquire a redação:

$$
\underset{2}{\chi_{1}}=\frac{\Lambda_{\mathrm{N}, 2}}{\Lambda_{1}}=\frac{p}{\sqrt{2} \pi}\left(\frac{\sqrt{\left.1+\gamma^{2} \mp \sqrt{\left(1+\gamma^{2}\right)^{2}-4 \gamma^{2}\left[1-\left(\frac{p}{2 \pi \delta \gamma}\right)^{2}\right.}\right]}}{1-\gamma^{2} \mp \sqrt{\left(1+\gamma^{2}\right)^{2}-4 \gamma^{2}\left[1-\left(\frac{p}{2 \pi \delta \gamma}\right)^{2}\right]}}\right) \frac{D_{1}-D_{2} \cos \left(\alpha_{N, 2} L\right)}{D_{3}^{*} \operatorname{sen}\left(\alpha_{N, 2} L\right)}
$$

A figura 4.8 ilustra o comportamento de (4.43) para $\gamma=1.4$ e $Z=1$.

a) $x$

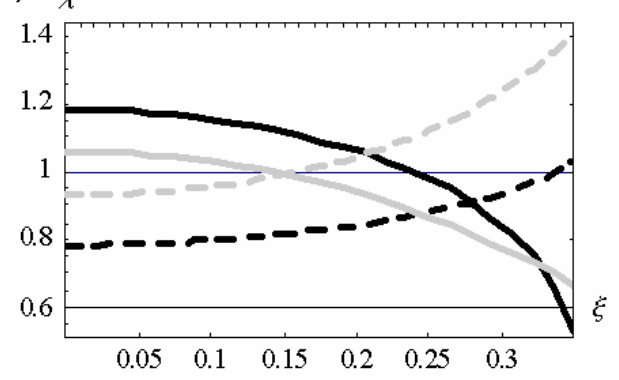

b) $x$

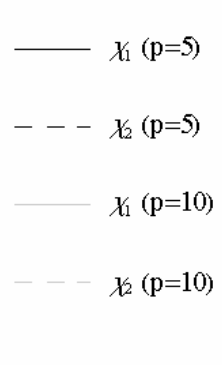

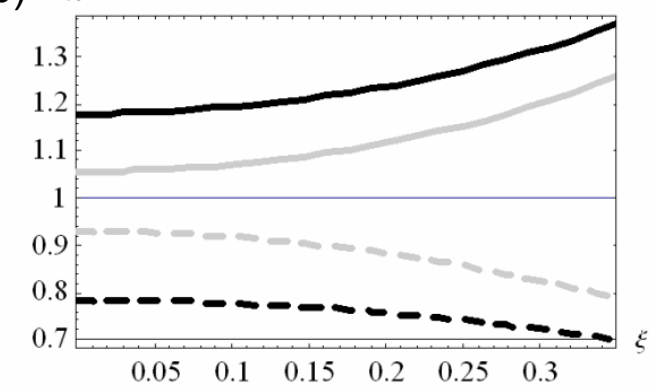

Figura 4.8 - Curva $\chi$ da primeira e da segunda freqüência espacial para casos com a) $\beta=1 / 4$ e b) $\beta=0$.

\subsection{CÁLCULO DA REFLEXÃO ESPÚRIA}

O estudo das reflexões espúrias no elemento de viga de Timoshenko é bastante similar ao realizado no caso do elemento de barra [LAIER (2002), NORONHA (2004)]]. No caso da flexão, devido à presença de um par de ondas com movimentos acoplados, uma perturbação incidente no nó j (com amplitude $\mathrm{1}_{1}$ ) gera quatro novas ondas, dois pares de ondas transmitidas (com amplitudes $\tau_{1} \mathrm{e}$ $\tau_{2}$ ) e outros dois de ondas refletidas (com amplitudes $\kappa_{1}$ e $\kappa_{2}$ ), conforme mostra a figura 4.9; uma vez que no nó genérico j há duas equações de compatibilidade a serem atendidas (igualdade de deslocamento transversal e de rotação do nó j para ambos elementos envolvidos), mais duas equações de equilíbrio, sendo uma de forças transversais e outra de momento fletor. 


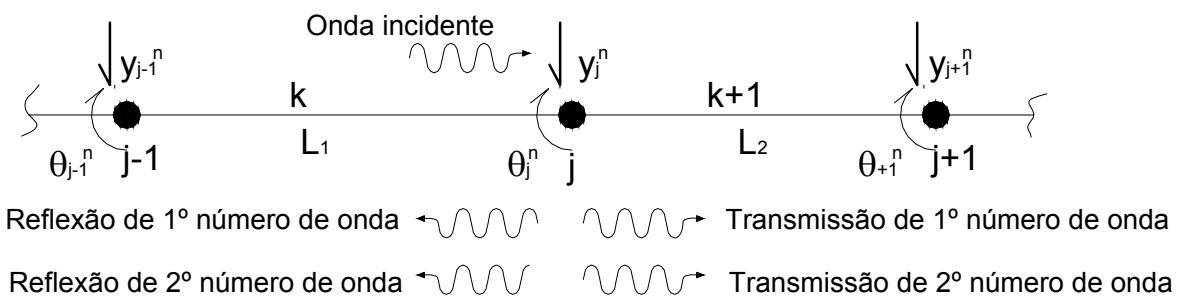

Figura 4.9 - Ondas envolvidas no fenômeno espúrio.

Relembrando a relação $\psi=L_{2} / L_{1}$, as relações entre os parâmetros adimensionais dos elementos são:

$$
\delta_{2}=\frac{1}{\psi} \delta_{1} \quad ; \quad \Rightarrow p_{2}=\frac{1}{\psi} p_{1} ; \quad \Phi_{2}=\frac{1}{\psi^{2}} \Phi_{1}
$$

Conferindo-se às matrizes de massa e de rigidez referentes ao sistema de equações uma forma adimensional do tipo:

$$
\begin{gathered}
{\left[\mathrm{M}_{1}\right]=\Psi_{\mathrm{M}, 1}\left(\left[\begin{array}{cccc}
\mathrm{m}_{3} & -\mathrm{m}_{4} & \mathrm{~m}_{1} & -\mathrm{m}_{2} \\
\mathrm{~m}_{4} & \mathrm{~m}_{6} & -\mathrm{m}_{2} & \mathrm{~m}_{5}
\end{array}\right]+\delta_{1}^{2}\left[\begin{array}{cccc}
-\mathrm{m}_{7} & -\mathrm{m}_{8} & \mathrm{~m}_{7} & -\mathrm{m}_{8} \\
\mathrm{~m}_{8} & \mathrm{~m}_{10} & -\mathrm{m}_{8} & \mathrm{~m}_{9}
\end{array}\right]\right)} \\
{\left[\mathrm{M}_{2}\right]=\Psi_{\mathrm{M}, 2}\left(\left[\begin{array}{cccc}
\mathrm{m}_{1} & \mathrm{~m}_{2} & \mathrm{~m}_{3} & \mathrm{~m}_{4} \\
\mathrm{~m}_{2} \psi & \mathrm{m}_{5} \psi & -\mathrm{m}_{4} \psi & \mathrm{m}_{6} \psi
\end{array}\right]+\frac{\delta_{1}^{2}}{\psi^{2}}\left[\begin{array}{cccc}
\mathrm{m}_{7} & \mathrm{~m}_{8} & -\mathrm{m}_{7} & \mathrm{~m}_{8} \\
\mathrm{~m}_{8} \psi & \mathrm{m}_{9} \psi & -\mathrm{m}_{8} \psi & \mathrm{m}_{10} \psi
\end{array}\right]\right)(4,4)} \\
{\left[\mathrm{K}_{1}\right]=\Psi_{\mathrm{K}, 1}^{*}\left[\begin{array}{cccc}
-12 & -6 & 12 & -6 \\
6 & 2-\Phi_{1} & -6 & 4+\Phi_{1}
\end{array}\right]} \\
{\left[\mathrm{K}_{2}\right]=\Psi_{\mathrm{K}, 2}^{*}\left[\begin{array}{cccc}
12 & 6 & -12 \\
6 \psi\left(4+\frac{1}{\theta^{2}} \Phi_{1}\right) & -6 \psi & \psi\left(2-\frac{1}{\theta^{2}} \Phi_{1}\right)
\end{array}\right]} \\
\Psi_{\mathrm{M}, 1}=\frac{1}{1+\Phi_{1}} \quad ; \quad \Psi_{\mathrm{M}, 2}=\frac{\psi\left(1+\Phi_{1}\right)}{\left(1+\frac{1}{\psi^{2}} \Phi_{1}\right)^{2}} \\
\Psi_{\mathrm{K}, 1}^{*}=\mathrm{p}_{1}^{2} \delta_{1}^{2} \xi^{2} \\
\Psi_{\mathrm{K}, 2}^{*}=\frac{p_{1}^{2} \delta_{1}^{2} \xi^{2}\left(1+\Phi_{1}\right)}{\psi^{3}\left(1+\frac{1}{\psi^{2}} \Phi_{1}\right)}
\end{gathered}
$$

tem-se que as ondas descritas na figura 4.4 implicam em vetores de deslocamentos análogos aos tratados na equação (2.33) do caso uniaxial, porém 
com o vetor de deslocamentos $\left\{u_{j}^{*}\right\}$ contendo ambos os números de onda. Assim sendo, os movimentos resultantes à esquerda e à direita são respectivamente dados por:

$$
\begin{aligned}
& \left\{u_{1}^{*}\right\}=\left\{u_{1}\right\}+\frac{\kappa_{1}}{l}\left\{u_{\mathrm{k} 1}\right\}+\frac{\kappa_{2}}{l}\left\{u_{\mathrm{k} 2}\right\} \\
& \left\{u_{2}^{*}\right\}=\frac{\tau_{1}}{\imath}\left\{u_{\tau 1}\right\}+\frac{\tau_{2}}{l}\left\{u_{\tau 2}\right\}
\end{aligned}
$$

Por outro lado, no sentido de se relacionar as amplitudes incógnitas das quatro ondas envolvidas com a incidente, inicialmente tem-se que os movimentos de translação y e rotação $\theta$ são do tipo:

$$
\begin{aligned}
& y=\left(C_{1} e^{i \alpha_{1} x}+C_{2} e^{i \alpha_{2} x}+C_{3} e^{-i \alpha_{1} x}+C_{4} e^{-i \alpha_{2} x}\right) e^{-i \omega t} \\
& \theta=\left(C^{\prime}{ }_{1} e^{i \alpha_{1} x}+C^{\prime}{ }_{2} e^{i \alpha_{2} x}+C^{\prime}{ }_{3} e^{-i \alpha_{1} x}+C^{\prime}{ }_{4} e^{-i \alpha_{2} x}\right) e^{-i \omega t}
\end{aligned}
$$

sendo que o atendimento das equações de equilíbrio (4.2) implica em:

$$
\begin{array}{lll}
C_{1}^{\prime}=C_{1}\left(\alpha_{1}-\frac{\rho A}{\phi \alpha_{1}} \omega^{2}\right) & C_{2}^{\prime}=C_{2}\left(\alpha_{2}-\frac{\rho A}{\phi \alpha_{2}} \omega^{2}\right) \\
C^{\prime}{ }_{3}=C_{3} i\left(\frac{\rho A}{\phi \alpha_{1}} \omega^{2}-\alpha_{1}\right) & ; & C_{4}^{\prime}=C_{4}\left(\frac{\rho A}{\phi \alpha_{2}} \omega^{2}-\alpha_{2}\right)
\end{array}
$$

Recorrendo-se agora à igualdade (4.13), resulta finalmente:

$$
\begin{array}{ll}
\mathrm{C}_{1}^{\prime}=\Lambda_{1} \mathrm{C}_{1} & ; \quad \mathrm{C}_{2}^{\prime}=\Lambda_{2} \mathrm{C}_{2} \\
\mathrm{C}_{3}^{\prime}=-\Lambda_{1} \mathrm{C}_{3} & ; \quad \mathrm{C}_{4}^{\prime}=-\Lambda_{2} \mathrm{C}_{4}
\end{array}
$$

Com isso os vetores de deslocamentos para as ondas envolvidas ficam expressos por: 


$$
\begin{aligned}
& \left\{u_{1}\right\}=\left\{\begin{array}{c}
e^{-i \alpha_{N, 1,1} L_{1}} \\
\Lambda_{N, 1,1} L_{1} e^{-i \alpha_{N, 1,1} L_{1}} \\
1 \\
\Lambda_{N, 1,1} L_{1}
\end{array}\right\} ;\left\{u_{k 1}\right\}=\left\{\begin{array}{c}
e^{i \alpha_{N, 1,1} L_{1}} \\
-\Lambda_{N, 1,1} L_{1} e^{i \alpha_{N, 1,1} L_{1}} \\
1 \\
-\Lambda_{N, 1,1} L_{1}
\end{array}\right\} ;\left\{u_{k 2}\right\}=\left\{\begin{array}{c}
e^{i \alpha_{N, 2,2} L_{1}} \\
-\Lambda_{N, 2,1} L_{1} e^{i \alpha_{N, 2, L} L_{1}} \\
1 \\
-\Lambda_{N, 2,1} L_{1}
\end{array}\right\} \\
& \left\{u_{\tau 1}\right\}=\left\{\begin{array}{c}
1 \\
\Lambda_{N, 1,2} L_{2} \\
e^{i \alpha_{N, 1,2} L_{2}} \\
\Lambda_{N, 1,2} L_{2} e^{i \alpha_{N, 1,2} L_{2}}
\end{array}\right\} ; \quad\left\{u_{\tau 2}\right\}=\left\{\begin{array}{c}
1 \\
\Lambda_{N, 2,2} L_{2} \\
e^{i \alpha_{N, 2,2} L_{2}} \\
\Lambda_{N, 2,2} L_{2} e^{i \alpha_{N, 2,2} L_{2}}
\end{array}\right\} \\
& \alpha_{N, 2, n}^{1, n} L_{n}=\arccos \left(\frac{\sigma_{2, n} \mp \sqrt{\sigma_{2, n}^{2}-4 \sigma_{1, n}}}{2}\right) \Lambda_{N, 2, n} L_{n}=i \frac{D_{1, n}-D_{2, n} \cos \left(\alpha_{N, 2}^{1, n} L_{n}\right)}{D_{3, n} \operatorname{sen}\left(\alpha_{N, 2}^{1, n} L_{n}\right)}
\end{aligned}
$$

onde, como já visto, a relação entre as amplitudes da solução numérica do giro $\theta$ e do movimento transversal y do $\mathrm{j}^{\mathrm{a}}$ freqüência espacial no elemento $n$ é denotado pela simbologia $\Lambda_{\mathrm{N}, \mathrm{j}, \mathrm{n}}$. $\mathrm{O}$ fato de seu valor ser imaginário indica que a função que expressa o giro $\theta$ é defasada $90^{\circ}$ do campo de deslocamentos y. Os valores $D_{j, n} e$ $\sigma_{\mathrm{j}, \mathrm{n}}$ do elemento $\mathrm{n}$ são os dispostos em (4.34) e (4.36) respectivamente. Aplicando toda esta redação na equação de movimento integrada no tempo, chega-se a um sistema de duas equações e quatro incógnitas.

Finalmente, o segundo par de equações a serem atendidas são as já mencionadas equações de compatibilidade, quais sejam:

$$
\begin{aligned}
& 1+\frac{\kappa_{1}}{\imath}+\frac{\kappa_{2}}{\imath}=\frac{\tau_{1}}{\imath}+\frac{\tau_{2}}{\imath} \\
& \Lambda_{\mathrm{N}, 1,1}-\Lambda_{\mathrm{N}, 1,1} \frac{\kappa_{1}}{l}-\Lambda_{\mathrm{N}, 2,1} \frac{\kappa_{2}}{\imath}=\Lambda_{\mathrm{N}, 1,2} \frac{\tau_{1}}{\imath}+\Lambda_{\mathrm{N}, 2,2} \frac{\tau_{2}}{\imath}
\end{aligned}
$$

completando-se assim o sistema de equações a ser atendido, e com isso as quatro relações incógnitas $\frac{\kappa_{1}}{l}, \frac{k_{2}}{l}, \frac{\tau_{1}}{l} \mathrm{e} \frac{\tau_{2}}{l}$ podem ser determinadas.

Como ilustração do apresentado, os gráficos das figuras 4.10 exibem o módulo da relação entre as amplitudes refletidas e a incidente da primeira e da segunda freqüência espacial, para um caso com os os parâmetros adimensionais 
valendo $Z=1, \xi=0.02, \gamma=1.3$ e $\beta=1 / 4$; empregando-se discretizações espaciais com $p_{1}=3, p_{1}=5$ e $p_{1}=7$.
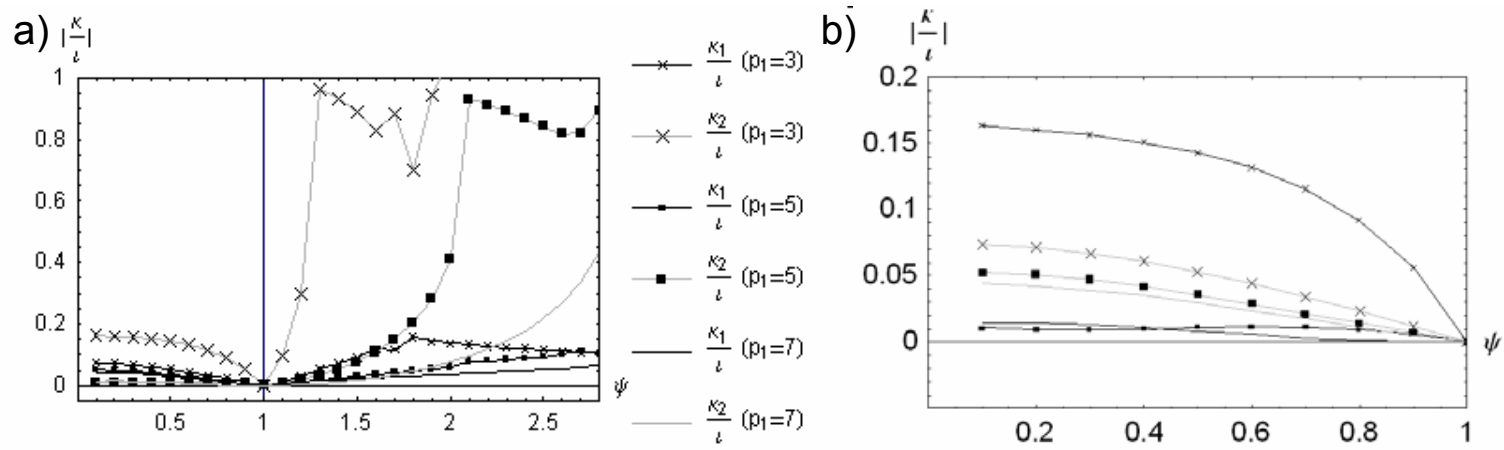

Figura 4.10 - Relação entre as amplitudes refletida e incidente para $Z=1$, $\xi=0.02, \gamma=1.3$ e $\beta=1 / 4$ com $p_{1}=3, p_{1}=5$ e $p_{1}=7$.

De acordo com os gráficos, as curvas têm características semelhantes, tanto para a primeira freqüência espacial quanto para a segunda, e que é considerável a contribuição do grau de refinamento da discretização espacial no efeito espúrio. Percebe-se ainda que, assim como no caso do elemento finito de barra, existe um limite finito assintótico associado à tendência $\psi$ a zero.

Naturalmente, o efeito espúrio atinge alto nível de atuação no sistema conforme $\psi$ cresce. Esta característica está associada à magnitude do erro referente à freqüência espacial. 


\section{$\underline{\text { Capítulo } 5}$}

\section{Algoritmos de Integração para Elementos de Viga}

\subsection{INTRODUÇÃO}

O bom desempenho dos integradores numéricos propostos para a formulação de elementos finitos na análise dinâmica de barras remete, em princípio, ao estudo de uma formulação análoga para elementos de viga. De fato, este tipo de elemento tem movimento ondulatório regido por duas equações diferenciais, que podem ser integradas através de uma combinação de termos de operadores discretos no tempo e no espaço.

Apresenta-se neste capítulo um integrador espacial simplificado, onde a matriz de massa resultante tem metade de seus termos nula, pois a aceleração angular não influencia no equilíbrio de forças transversais e a aceleração linear não interfere no equilíbrio de momentos. 


\subsection{FORMULACCÃO MEDIANTE INTEGRADOR ESPACIAL DO TIPO $\beta_{\mathrm{s}}$}

Conforme discutido anteriormente, o procedimento para encontrar a equação de diferenças relativa aos elementos de viga, segundo a teoria de Timoshenko, é análogo ao empregado no elemento de barra, a menos do fato de que o elemento finito de viga, como já sabido, obedece a duas equações diferenciais de equilíbrio. Nesta parte do estudo, introduz-se a formulação de um integrador espacial simplificado, capaz de elevar a ordem do erro de truncamento.

Como sabido, as equações diferenciais regentes do movimento ondulatório em questão são dadas por:

$$
\begin{aligned}
& \ddot{y}_{j}^{n}-c_{s}^{2} y_{j}^{n}{ }^{\prime \prime}+c_{s}^{2} \theta_{j}^{n}{ }^{\prime}=0 \\
& r^{2} \ddot{\theta}_{j}^{n}-c_{R}^{2} r^{2} \theta_{j}^{n \prime \prime}-c_{s}^{2} y_{j}^{n}+c_{s}^{2} \theta_{j}^{n}=0
\end{aligned}
$$

onde se considera a mesma notação do capítulo anterior.

De início, duas considerações interessantes precisam ser assinaladas. A primeira delas é que, em princípio, as equações de equilíbrio de forças (5.1a) e a de momentos (5.1b) não contêm respectivamente os termos correspondentes à aceleração angular e transversal da seção da viga. Este aspecto de desacoplamento verificado nas equações de equilíbrio implica numa matriz de massa que pode ser composta em sua metade por termos nulos, contribuindo assim para um menor esforço computacional.

Outra ocorrência que vale citar é que, a menos do sinal invertido, o fator da primeira derivada do giro em (5.1a) é idêntico ao da primeira derivada do deslocamento transversal em (5.1b). Conseqüentemente, tal fato implica (justifica) na simetria da matriz de rigidez.

Em complemento ao expresso na equação (3.18), buscou-se formular uma adequada expressão para denotar a equação de diferenças referente à primeira derivada de cada grau de liberdade, presente nas equações de equilíbrio de ações. Abreviando algumas passagens matemáticas do método de Newmark, a eliminação do termo referente à aceleração permite escrever: 


$$
\frac{1}{2 L} \stackrel{s}{\Gamma} u_{j}^{n}=\left(1+\beta_{s} \stackrel{s}{\nabla}\right) u_{j}^{n}
$$

onde o operador $\stackrel{s}{\Gamma}$ tem a seguinte formulação:

$$
\stackrel{s}{\Gamma} X_{j}^{n}=X_{j+1}^{n}-X_{j-1}^{n}
$$

que consiste numa simples diferença.

Multiplicando as equações contidas em (5.1) por $1+\beta_{S} \stackrel{s}{\nabla}$, consegue-se um sistema semi-discretizado no espaço, com o formato adimensional, qual seja:

$$
\begin{aligned}
& \gamma^{2} \mathrm{~T}^{2}\left(1+\beta_{\mathrm{s}} \stackrel{\mathrm{s}}{\nabla}\right) \ddot{\mathrm{y}}_{\mathrm{j}}^{\mathrm{n}}-\mathrm{p}^{2} \xi^{2} \stackrel{\mathrm{s}}{\nabla} \mathrm{y}_{\mathrm{j}}^{\mathrm{n}}+\frac{1}{2} \mathrm{p}^{2} \xi^{2} \stackrel{\mathrm{s}}{\Gamma}\left(\theta_{\mathrm{j}}^{\mathrm{n}} \mathrm{L}\right)=0 \\
& \delta^{2} \gamma^{2} \mathrm{~T}^{2}\left(1+\beta_{\mathrm{s}} \stackrel{\mathrm{s}}{\nabla}\right)\left(\ddot{\theta}_{\mathrm{j}}^{\mathrm{n}} \mathrm{L}\right)-\frac{1}{2} \mathrm{p}^{2} \xi^{2} \stackrel{\mathrm{s}}{\Gamma} \mathrm{y}_{\mathrm{j}}^{\mathrm{n}}+\left[\mathrm{p}^{2} \xi^{2}\left(1+\beta_{\mathrm{s}} \stackrel{\mathrm{s}}{\nabla}\right)-\mathrm{p}^{2} \gamma^{2} \delta^{2} \xi^{2} \stackrel{\mathrm{s}}{\nabla}\right]\left(\theta_{j}^{\mathrm{n}} \mathrm{L}\right)=0
\end{aligned}
$$

que consiste numa equação diferencial (tempo) de diferenças (espaço).

Explicitando-se o vetor de deslocamentos nodais $\{U\}$, que reúne os graus de liberdade que afetam o equilíbrio do nó j, na forma dimensional que se segue:

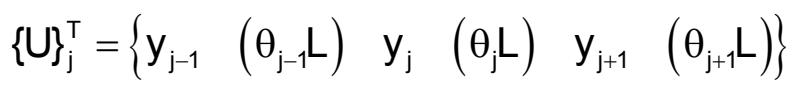

pode-se, pois, resumir os operadores envolvidos na forma:

$$
\begin{aligned}
& \stackrel{\mathrm{s}}{\nabla} \mathrm{y}_{\mathrm{j}}=\left\{\begin{array}{llllll}
1 & 0 & -2 & 0 & 1 & 0
\end{array}\right\}\{\mathrm{U}\}_{\mathrm{j}} ; \quad \stackrel{\mathrm{s}}{\nabla}\left(\theta_{\mathrm{j}} \mathrm{L}\right)=\left\{\begin{array}{llllll}
0 & 1 & 0 & -2 & 0 & 1
\end{array}\right\}\{\mathrm{U}\}_{\mathrm{j}} \\
&\left(1+\beta_{\mathrm{s}} \stackrel{\mathrm{s}}{\nabla}\right) \mathrm{y}_{\mathrm{j}}=\left\{\begin{array}{llllll}
\beta_{\mathrm{s}} & 0 & 1-2 \beta_{\mathrm{s}} & 0 & \beta_{\mathrm{s}} & 0
\end{array}\right\}\{\mathrm{U}\}_{\mathrm{j}} \\
&\left(1+\beta_{\mathrm{s}} \stackrel{\mathrm{s}}{\nabla}\right)\left(\theta_{\mathrm{j}} \mathrm{L}\right)=\left\{\begin{array}{lllllll}
0 & \beta_{\mathrm{s}} & 0 & 1-2 \beta_{\mathrm{s}} & 0 & \beta_{\mathrm{s}}
\end{array}\right\}\{\mathrm{U}\}_{\mathrm{j}} \\
& \stackrel{\mathrm{s}}{\Gamma} \mathrm{y}_{\mathrm{j}}=\left\{\begin{array}{llllll}
-1 & 0 & 0 & 0 & 1 & 0
\end{array}\right\}\{\mathrm{U}\}_{\mathrm{j}} ; \quad \stackrel{\mathrm{s}}{\Gamma}\left(\theta_{\mathrm{j}} \mathrm{L}\right)=\left\{\begin{array}{llllll}
0 & -1 & 0 & 0 & 0 & 1
\end{array}\right\}\{\mathrm{U}\}_{\mathrm{j}}
\end{aligned}
$$

Com isso, as equações (5.4) ganham, em forma matricial, a seguinte redação: 


$$
\left[\overline{\mathrm{M}}_{\mathrm{G}}\right]\{\ddot{\mathrm{U}}\}_{\mathrm{j}}+\left[\overline{\mathrm{K}}_{\mathrm{G}}\right]\{\mathrm{U}\}_{\mathrm{j}}=\{0\}
$$

com:

$$
\begin{aligned}
& {\left[\overline{\mathrm{M}}_{\mathrm{G}}\right]=\gamma^{2}\left[\begin{array}{cccccc}
\beta_{\mathrm{s}} & 0 & 1-2 \beta_{\mathrm{s}} & 0 & \beta_{\mathrm{s}} & 0 \\
0 & \delta^{2} \beta_{\mathrm{s}} & 0 & \delta^{2}\left(1-2 \beta_{\mathrm{s}}\right) & 0 & \delta^{2} \beta_{\mathrm{s}}
\end{array}\right]} \\
& {\left[\overline{\mathrm{K}}_{\mathrm{G}}\right]=\frac{\mathrm{p}^{2} \xi^{2}}{\mathrm{~T}^{2}}\left[\begin{array}{ccccccc}
-1 & -\frac{1}{2} & 2 & 0 & -1 & \frac{1}{2} \\
\frac{1}{2} & \beta_{\mathrm{s}}-\gamma^{2} \delta^{2} & 0 & 1-2 \beta_{\mathrm{s}}+2 \gamma^{2} \delta^{2} & -\frac{1}{2} & \beta_{\mathrm{s}}-\gamma^{2} \delta^{2}
\end{array}\right]}
\end{aligned}
$$

Estas matrizes de contribuição para o equilíbrio do nó genérico j estão associadas às matrizes locais, dadas por:

$$
\begin{aligned}
& {[\overline{\mathrm{M}}]=\left[\begin{array}{cccc}
\frac{1}{2}-\beta_{\mathrm{s}} & 0 & \beta_{\mathrm{s}} & 0 \\
0 & \delta^{2}\left(\frac{1}{2}-\beta_{\mathrm{s}}\right) & 0 & \delta^{2} \beta_{\mathrm{s}} \\
\beta_{\mathrm{s}} & 0 & \frac{1}{2}-\beta_{\mathrm{s}} & 0 \\
0 & \delta^{2} \beta_{\mathrm{s}} & 0 & \delta^{2}\left(\frac{1}{2}-\beta_{\mathrm{s}}\right)
\end{array}\right]} \\
& {[\overline{\mathrm{K}}]=\frac{\mathrm{p}^{2} \xi^{2} \delta^{2}}{\Phi T^{2}}\left[\begin{array}{cccc}
12 & 6 & -12 & 6 \\
6 & 6-12 \beta_{\mathrm{s}}+\Phi & -6 & 12 \beta_{\mathrm{s}}-\Phi \\
-12 & -6 & 12 & -6 \\
6 & 12 \beta_{\mathrm{s}}-\Phi & -6 & 6-12 \beta_{\mathrm{s}}+\Phi
\end{array}\right]}
\end{aligned}
$$

Complementarmente, multiplicando-se ambas matrizes de (5.9) pela inércia linear $\rho A L$ têm-se um resultado que é compatível com o da modelagem clássica (vibração livre não amortecida). A saber, uma vez que $\Phi \cong \Phi+1$, quando $\beta_{\text {s }}$ assume o valor de $1 / 6$ chega-se à mesma matriz de rigidez obtida pelo método do mínimo potencial total com função de forma linear.

Vale ressaltar que as matrizes redigidas em (5.9) já estão com seus elementos adimensionalizados. Contudo, à primeira vista, o fator comum da matriz de rigidez parece não seguir esta regra por conter um termo dimensional de tempo (T). Todavia, tal parâmetro é eliminado ora pela integração temporal no caso de uma análise em termos de deslocamentos, ora pelo numerador do termo de discretização temporal $\xi$ em uma análise modal. 


\subsection{FORMULAČ̃̃O GENERALIZADA}

A formulação do problema da minimização da dispersão da velocidade numérica em sistemas discretizados é aqui buscada conferindo-se a seguinte escrita para as matrizes de massa e de rigidez:

$$
[\mathrm{M}]=\left[\begin{array}{cccc}
\mathrm{m}_{2} & 0 & \mathrm{~m}_{3} & 0 \\
0 & \overline{\mathrm{M}}_{2} & 0 & \overline{\mathrm{M}}_{3} \\
\mathrm{~m}_{1} & 0 & \mathrm{~m}_{2} & 0 \\
0 & \overline{\mathrm{M}}_{1} & 0 & \overline{\mathrm{M}}_{2}
\end{array}\right] \quad ; \quad[\mathrm{K}]=\left[\begin{array}{cccc}
\mathrm{k}_{2} & \mathrm{~K}_{2} & \mathrm{k}_{3} & \mathrm{~K}_{3} \\
\overline{\mathrm{k}}_{2} & \overline{\mathrm{K}}_{2} & \overline{\mathrm{k}}_{3} & \overline{\mathrm{K}}_{3} \\
\mathrm{k}_{1} & \mathrm{~K}_{1} & \mathrm{k}_{2} & -\mathrm{K}_{2} \\
\overline{\mathrm{k}}_{1} & \overline{\mathrm{K}}_{1} & -\overline{\mathrm{k}}_{2} & \overline{\mathrm{K}}_{2}
\end{array}\right]
$$

as quais implicam nas seguintes equações de equilíbrio de forças e de momentos, respectivamente:

$$
\begin{aligned}
& \frac{m}{\nabla} \ddot{y}_{j}+\stackrel{k}{\nabla} y_{j}+\stackrel{k}{\nabla} \theta_{j}=0 \\
& \stackrel{\bar{M}}{\nabla} \ddot{\theta}_{j}+\stackrel{\bar{k}}{\nabla} y_{j}+\stackrel{\bar{K}}{\nabla} \theta_{j}=0
\end{aligned}
$$

onde, novamente, os operadores $\bar{\nabla}$ e $\breve{\nabla}$ significam:

$$
\begin{aligned}
& \stackrel{x}{\nabla} z_{j}=X_{1} z_{j-1}+2 X_{2} z_{j}+X_{3} z_{j+1} \\
& \stackrel{x}{\nabla} z_{j}=X_{1} z_{j-1}+X_{3} z_{j+1}
\end{aligned}
$$

que consistem em simples operações de diferença.

Assumindo-se um campo de deslocamentos no formato exponencial como exposto em (2.8), pode-se reescrever as equações (5.11) convenientemente na forma:

$$
\begin{aligned}
& \ddot{y}_{j}+\frac{k_{1} e^{-\alpha_{N} L}+2 k_{2}+k_{3} e^{\alpha_{N} L}}{m_{1} e^{-\alpha_{N} L}+2 m_{2}+m_{3} e^{\alpha_{N} L}} y_{j}+\frac{K_{1} e^{-\alpha_{N} L}+K_{3} e^{\alpha_{N} L}}{m_{1} e^{-\alpha_{N} L}+2 m_{2}+m_{3} e^{\alpha_{N} L}} \theta_{j}=0 \\
& \ddot{\theta}_{j}+\frac{\bar{k}_{1} e^{-\alpha_{N} L}+\bar{k}_{3} e^{\alpha_{N} L}}{\bar{M}_{1} e^{-\alpha_{N} L}+2 \bar{M}_{2}+\bar{M}_{3} e^{\alpha_{N} L}} y_{j}+\frac{\bar{K}_{1} e^{-\alpha_{N} L}+2 \bar{K}_{2}+\bar{K}_{3} e^{\alpha_{N} L}}{\bar{M}_{1} e^{-\alpha_{N} L}+2 \bar{M}_{2}+\bar{M}_{3} e^{\alpha_{N} L}} \theta_{j}=0
\end{aligned}
$$


Facilitando-se assim sua comparação com a expressão advinda da formulação teórica:

$$
\begin{aligned}
& \ddot{y}(x, t)+\alpha^{2} c_{S}^{2} y(x, t)+i \alpha c_{S}^{2} \theta(x, t)=0 \\
& \ddot{\theta}(x, t)-i \alpha \frac{c_{S}^{2}}{r^{2}} y(x, t)+\left(\alpha^{2} c_{R}^{2}+\frac{c_{S}^{2}}{r^{2}}\right) \theta(x, t)=0
\end{aligned}
$$

Cria-se desta maneira um sistema algébrico não linear com o objetivo de convergir com a maior ordem de erro possível às seguintes tendências:

$$
\begin{aligned}
& \frac{\mathrm{k}_{1} \mathrm{e}^{-\alpha_{N} L}+2 \mathrm{k}_{2}+\mathrm{k}_{3} \mathrm{e}^{\alpha_{N} L}}{\mathrm{~m}_{1} \mathrm{e}^{-\alpha_{N} L}+2 \mathrm{~m}_{2}+\mathrm{m}_{3} \mathrm{e}^{\alpha_{N} L}} \rightarrow \alpha_{N}^{2} c_{S}^{2} \\
& \frac{\mathrm{K}_{1} \mathrm{e}^{-\alpha_{N} L}+\mathrm{K}_{3} \mathrm{e}^{\alpha_{N} L}}{\mathrm{~m}_{1} \mathrm{e}^{-\alpha_{N} L}+2 \mathrm{~m}_{2}+\mathrm{m}_{3} \mathrm{e}^{\alpha_{N} L}} \rightarrow i \alpha_{N} c_{S}^{2} \\
& \frac{\overline{\mathrm{k}}_{1} \mathrm{e}^{-\alpha_{N} L}+\overline{\mathrm{k}}_{3} \mathrm{e}^{\alpha_{N} L}}{\overline{\mathrm{M}}_{1} \mathrm{e}^{-\alpha_{N} L}+2 \overline{\mathrm{M}}_{2}+\overline{\mathrm{M}}_{3} \mathrm{e}^{\alpha_{N} L}} \rightarrow-i \alpha_{N} \frac{\mathrm{c}_{S}^{2}}{\mathrm{r}^{2}} \\
& \frac{\overline{\mathrm{K}}_{1} \mathrm{e}^{-\alpha_{N} L}+2 \overline{\mathrm{K}}_{2}+\overline{\mathrm{K}}_{3} \mathrm{e}^{\alpha_{N} L}}{\overline{\mathrm{M}}_{1} \mathrm{e}^{-\alpha_{N} L}+2 \overline{\mathrm{M}}_{2}+\overline{\mathrm{M}}_{3} \mathrm{e}^{\alpha_{N} L}} \rightarrow \alpha_{N}^{2} c_{\mathrm{R}}^{2}+\frac{\mathrm{c}_{S}^{2}}{\mathrm{r}^{2}}
\end{aligned}
$$

O fato de o par de expressões (5.15a) e (5.15b), bem como (5.15c) e $(5.15 d)$, possuírem variáveis em comum $\left(m_{j}\right.$ e $M_{j}$ respectivamente), restringe a liberdade de uma combinação de operadores, induzindo à preferência de uma ou outra expressão para a maximização da ordem de erro. Tomando-se a equação de movimento, para ambas as possibilidades, tem-se que a melhor combinação relativa à matriz de rigidez é dada por:

$$
\begin{aligned}
& \left\{\begin{array}{lll}
\mathrm{k}_{1} & 2 \mathrm{k}_{2} & \mathrm{k}_{3}
\end{array}\right\}=\frac{12 \mathrm{c}_{\mathrm{S}}^{2}}{\mathrm{~L}^{2}} \mathrm{~m}_{3}\left\{\begin{array}{lll}
-1 & 2 & -1
\end{array}\right\} \\
& \left\{\begin{array}{ll}
\mathrm{K}_{1} & \mathrm{~K}_{3}
\end{array}\right\}=\frac{6 \mathrm{c}_{\mathrm{S}}^{2}}{\mathrm{~L}} \mathrm{~m}_{3}\left\{\begin{array}{ll}
-1 & 1
\end{array}\right\}
\end{aligned}
$$


Além disso, duas possibilidades combinatórias existem para a matriz de massa:

$$
\left\{m_{1} 2 m_{2} \quad m_{3}\right\}=m_{3}\left\{\begin{array}{lll}
1 & 10 & 1
\end{array}\right\}
$$

ou

$$
\left\{\begin{array}{lll}
m_{1} & 2 m_{2} & m_{3}
\end{array}\right\}=m_{3}\left\{\begin{array}{lll}
1 & 4 & 1
\end{array}\right\}
$$

A primeira delas, designada de acordo com a igualdade (5.17a), fornece melhor aproximação do fator de $\mathrm{y}_{\mathrm{j}}$, enquanto que a segunda expressão de (5.17) retorna um melhor fator para $\theta_{\mathrm{j}}$. Ou seja, a expansão em série de Taylor das funções presentes em (5.13a), tendo-se em vista o expresso em (5.17a), permite a redação:

$$
\ddot{y}_{j}+\left(\alpha_{N}^{2} c_{S}^{2}+O\left[L^{4}\right]\right) y_{j}+\left(i \alpha_{N} c_{S}^{2}-\frac{1}{12} \alpha_{N}^{3} c_{S}^{2} L^{2}+O\left[L^{4}\right]\right) \theta_{j}=0
$$

e, procedendo-se de modo similar com a equação (5.13a), tendo se em vista a redação de $(5.17 b)$, resulta:

$$
\ddot{y}_{j}+\left(\alpha_{N}^{2} c_{S}^{2}+\frac{1}{12} \alpha_{N}^{4} c_{S}^{2} L^{2}+O\left[L^{4}\right]\right) y_{j}+\left(i \alpha_{N} c_{S}^{2}+O\left[L^{4}\right]\right) \theta_{j}=0
$$

$\mathrm{Na}$ equação de equilíbrio de momentos, procedendo-se as combinações que se seguem:

$$
\begin{aligned}
& \left\{\begin{array}{ll}
\overline{\mathrm{k}}_{1} & \overline{\mathrm{k}}_{3}
\end{array}\right\}=\frac{3 \mathrm{c}_{\mathrm{S}}^{2}}{\mathrm{~L}^{3} \delta^{2}} \overline{\mathrm{M}}_{3}\left\{\begin{array}{ll}
1 & -1
\end{array}\right\} \\
& \left\{\begin{array}{lll}
\overline{\mathrm{K}}_{1} & 2 \overline{\mathrm{K}}_{2} & \overline{\mathrm{K}}_{3}
\end{array}\right\}=\frac{\mathrm{c}_{\mathrm{s}}^{2}}{\mathrm{~L}^{2}} \overline{\mathrm{M}}_{3}\left\{\frac{1}{\delta^{2}}-6 \gamma^{2} \quad 2\left(6 \gamma^{2}+\frac{2}{\delta^{2}}\right) \frac{1}{\delta^{2}}-6 \gamma^{2}\right\} \\
& \left\{\begin{array}{lll}
\overline{\mathrm{M}}_{1} & 2 \overline{\mathrm{M}}_{2} & \overline{\mathrm{M}}_{3}
\end{array}\right\}=\overline{\mathrm{M}}_{3}\left\{\begin{array}{lll}
1 & 4 & 1
\end{array}\right\}
\end{aligned}
$$

chega-se a uma nova e oportuna redação: 


$$
\ddot{\theta}_{j}+\left(-i \alpha \frac{c_{S}^{2}}{r^{2}}+O\left[L^{4}\right]\right) y_{j}+\left(\alpha^{2} c_{R}^{2}+\frac{c_{S}^{2}}{r^{2}}+\frac{1}{12} \alpha_{N}^{4} c_{S}^{2} L^{2}+O\left[L^{4}\right]\right) \theta_{j}=0
$$

Já as combinações:

$$
\begin{aligned}
& \left\{\begin{array}{ll}
\overline{\mathrm{k}}_{1} & \overline{\mathrm{k}}_{3}
\end{array}\right\}=\frac{6 \mathrm{c}_{\mathrm{s}}^{2}}{\mathrm{~L}^{3} \delta^{2}} \overline{\mathrm{M}}_{3}\left\{\begin{array}{ll}
1 & -1
\end{array}\right\} \\
& \left\{\begin{array}{lll}
\overline{\mathrm{K}}_{1} & 2 \overline{\mathrm{K}}_{2} & \overline{\mathrm{K}}_{3}
\end{array}\right\}=\frac{\mathrm{C}_{\mathrm{S}}^{2}}{\mathrm{~L}^{2}} \overline{\mathrm{M}}_{3}\left\{\frac{1}{\delta^{2}}-12 \gamma^{2} \quad 2\left(12 \gamma^{2}+\frac{5}{\delta^{2}}\right) \quad \frac{1}{\delta^{2}}-6 \gamma^{2}\right\} \\
& \left\{\begin{array}{lll}
\bar{M}_{1} & 2 \bar{M}_{2} & \bar{M}_{3}
\end{array}\right\}=\bar{M}_{3}\left\{\begin{array}{lll}
1 & 10 & 1
\end{array}\right\}
\end{aligned}
$$

implicam em:

$$
\ddot{\theta}_{j}+\left(-i \alpha \frac{c_{S}^{2}}{r^{2}}+\frac{1}{12} \frac{\alpha_{N}^{3} c_{S}^{2}}{r^{2}} L^{2}+O\left[L^{4}\right]\right) y_{j}+\left(\alpha^{2} c_{R}^{2}+\frac{c_{S}^{2}}{r^{2}}+O\left[L^{4}\right]\right) \theta_{j}=0
$$

A determinação dos parâmetros restantes, quais sejam, $m_{3}, \bar{M}_{3}, K_{2}$ e $\bar{k}_{2}$ pode ser feita através da imposição do atendimento das condições ditas gerais, que consistem em determinante nulo e simetria para a matriz de rigidez e que a soma dos termos da primeira e terceira linhas da matriz de massa reproduzam a inércia do elemento como corpo rígido.

Matematicamente, ambas as matrizes de rigidez aqui obtidas contemplam um sistema não trivial de solução se, e somente se:

$$
\mathrm{K}_{2}=\left(\frac{\mathrm{C}_{\mathrm{S}}^{2}}{2 \mathrm{~L}}\right) \frac{3 \frac{\mathrm{C}_{\mathrm{S}}^{2}}{\mathrm{~L}}-2 \overline{\mathrm{K}}_{2}}{\frac{\mathrm{c}_{\mathrm{S}}^{2}}{\mathrm{~L}}+2 \overline{\mathrm{K}}_{2}}
$$

e a condição de simetria implica em:

$$
\mathrm{K}_{2}=\overline{\mathrm{k}}_{2}=\frac{\mathrm{c}_{\mathrm{S}}^{2}}{2 \mathrm{~L}}
$$

A condição para definir os parâmetros restantes da matriz de massa é dada pela inércia de corpo rígido, conforme já referida no capítulo 3 , quando da análise 
do elemento simples de barra. Realmente, quando o corpo apresenta-se em movimento de corpo rígido com aceleração constante na direção axial, a resultante de forças deve ser igual à massa multiplicada pela aceleração. Assim, como a aceleração é a mesma para os dois graus de liberdade, o somatório dos termos da matriz de massa deve ser igual à massa total do elemento.

Entretanto, para se aplicar tal condição no elemento de viga aqui abordado, é necessário um exame mais detalhado. Nesse sentido, seja o caso de uma viga transladando com aceleração constante (denotada pela letra a) na direção perpendicular ao seu eixo. Nesta circunstância, não existe aceleração angular nos nós, fato que conduz ao seguinte vetor de ações nodais:

$$
\left[\begin{array}{cccc}
\mathrm{m}_{2} & 0 & \mathrm{~m}_{3} & 0 \\
0 & \bar{M}_{2} & 0 & \bar{M}_{3} \\
\mathrm{~m}_{1} & 0 & \mathrm{~m}_{2} & 0 \\
0 & \bar{M}_{1} & 0 & \bar{M}_{2}
\end{array}\right]\left\{\begin{array}{l}
a \\
0 \\
a \\
0
\end{array}\right\}=\left\{\begin{array}{c}
\mathrm{m}_{2}+\mathrm{m}_{3} \\
0 \\
\mathrm{~m}_{1}+\mathrm{m}_{2} \\
0
\end{array}\right\}
$$

Implicando-se que o somatório dos termos de cada uma das igualdades (5.17) deve ser igual à massa total do elemento.

De forma análoga, condicionando um elemento isolado a um giro segundo um eixo normal ao plano da viga, e localizado no seu centro geométrico, concluise que o somatório dos termos de cada uma das igualdades (5.22) deve ser igual à $\rho l$.

Pode-se então ser admitido quatro combinações de matrizes, uma vez que existem duas combinações possíveis para se aproximar cada equação. Porém, por simplificação, admite-se apenas dois casos: um de ordem elevada para os fatores de y e outro para os de $\theta$, denotados pelas letras $Y$ e $\Theta$. Tendo-se em conta uma formulação adimensional, dividem-se todas as equações do sistema pelo produto $\rho A L$, bem como dividem-se também as equações de equilíbrio de momentos pelo comprimento L, chegando-se em: 


$$
\begin{aligned}
& {\left[\mathrm{M}_{\mathrm{Y}}\right]=\left[\begin{array}{cccc}
\frac{5}{12} & 0 & \frac{1}{12} & 0 \\
0 & \frac{\delta^{2}}{3} & 0 & \frac{\delta^{2}}{6} \\
\frac{1}{12} & 0 & \frac{5}{12} & 0 \\
0 & \frac{\delta^{2}}{6} & 0 & \frac{\delta^{2}}{3}
\end{array}\right]} \\
& {\left[\mathrm{K}_{\mathrm{Y}}\right]=\left[\begin{array}{cccc}
\mathrm{p}^{2} \gamma^{2} & \frac{1}{2} \mathrm{p}^{2} \gamma^{2} & -\mathrm{p}^{2} \gamma^{2} & \frac{1}{2} \mathrm{p}^{2} \gamma^{2} \\
\frac{1}{2} \mathrm{p}^{2} \gamma^{2} & \frac{1}{3} \mathrm{p}^{2}\left(\gamma^{2}+3 \delta^{2}\right) & -\frac{1}{2} \mathrm{p}^{2} \gamma^{2} & \frac{\delta^{2}}{6} \\
-\mathrm{p}^{2} \gamma^{2} & -\frac{1}{2} \mathrm{p}^{2} \gamma^{2} & \mathrm{p}^{2} \gamma^{2} & -\frac{1}{2} \mathrm{p}^{2} \gamma^{2} \\
\frac{1}{2} \mathrm{p}^{2} \gamma^{2} & \frac{\delta^{2}}{6} & -\frac{1}{2} \mathrm{p}^{2} \gamma^{2} & \frac{1}{3} \mathrm{p}^{2}\left(\gamma^{2}+3 \delta^{2}\right)
\end{array}\right]}
\end{aligned}
$$

ou

$$
\begin{aligned}
& {\left[\mathrm{M}_{\Theta}\right]=\left[\begin{array}{cccc}
\frac{1}{3} & 0 & \frac{1}{6} & 0 \\
0 & \frac{5 \delta^{2}}{12} & 0 & \frac{\delta^{2}}{12} \\
\frac{1}{6} & 0 & \frac{1}{3} & 0 \\
0 & \frac{\delta^{2}}{12} & 0 & \frac{5 \delta^{2}}{12}
\end{array}\right]} \\
& {\left[\mathrm{K}_{\Theta}\right]=\left[\begin{array}{cccc}
\mathrm{p}^{2} \gamma^{2} & \frac{1}{2} \mathrm{p}^{2} \gamma^{2} & -\mathrm{p}^{2} \gamma^{2} & \frac{1}{2} \mathrm{p}^{2} \gamma^{2} \\
\frac{1}{2} \mathrm{p}^{2} \gamma^{2} & \frac{1}{12} \mathrm{p}^{2}\left(5 \gamma^{2}+12 \delta^{2}\right) & -\frac{1}{2} \mathrm{p}^{2} \gamma^{2} & \frac{\delta^{2}}{6} \\
-\mathrm{p}^{2} \gamma^{2} & -\frac{1}{2} \mathrm{p}^{2} \gamma^{2} & \mathrm{p}^{2} \gamma^{2} & -\frac{1}{2} \mathrm{p}^{2} \gamma^{2} \\
\frac{1}{2} \mathrm{p}^{2} \gamma^{2} & \frac{\delta^{2}}{6} & -\frac{1}{2} \mathrm{p}^{2} \gamma^{2} & \frac{1}{12} \mathrm{p}^{2}\left(5 \gamma^{2}+12 \delta^{2}\right)
\end{array}\right]}
\end{aligned}
$$

\subsection{RESULTADOS NUMÉRICOS}

Como exemplo de aplicação, seja o caso da discretização temporal segundo os parâmetros $\xi=0.05, \beta=1 / 12, \gamma=1.3$ e $Z=1$. Empregando-se as matrizes descritas em (5.9), as figuras 5.1, 5.2 e 5.3 comparam respectivamente o módulo de $\eta$, o de $\chi$ e o argumento (ângulo de fase) de $\eta$, para $\beta_{\mathrm{s}}=1 / 12$ e $\beta_{\mathrm{s}}=1 / 6$ em função do parâmetro de discretização espacial $p$.

Percebe-se que, embora a formulação com $\beta_{s}=1 / 12$ apresente melhores resultados em relação à freqüência espacial freqüência espacial, quando $\beta_{\mathrm{s}}$ vale 1/6 o coeficiente $\Lambda_{\mathrm{N}}$ (fator da amplitude de deslocamento transversal do problema discretizado) tem valor relativamente próximo do teórico na região inicial do domínio $\mathrm{p}$. Além disso, $\beta_{\mathrm{s}}=1 / 6$ tem menor zona de onda resultando evanescente. 
Em concordância com o estudo realizado na barra uniaxial, o fenômeno da onda evanescente é reduzido conforme $\beta_{\mathrm{s}}$ tende a $1 / 4$.

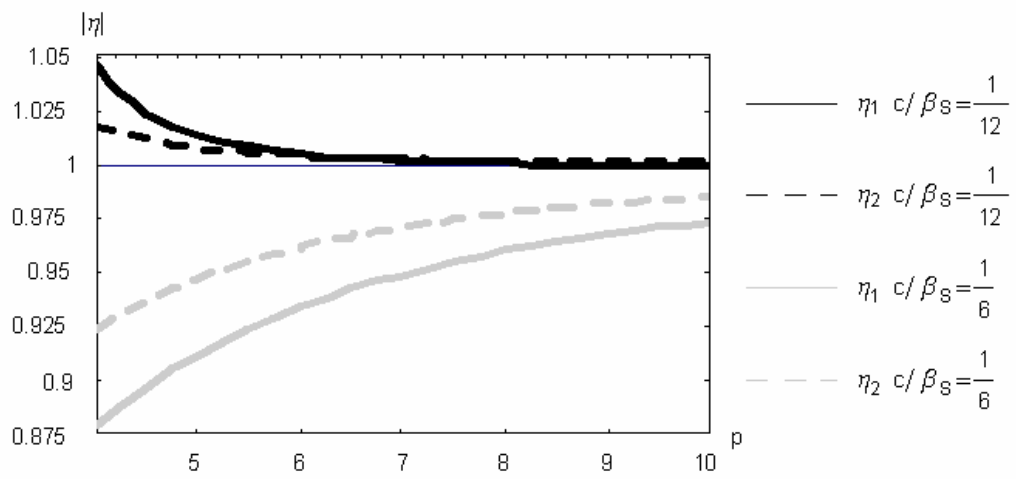

Figura 5.1 - Módulo de $\eta$ para $\beta_{\mathrm{s}}=1 / 12$ e $\beta_{\mathrm{s}}=1 / 6$.

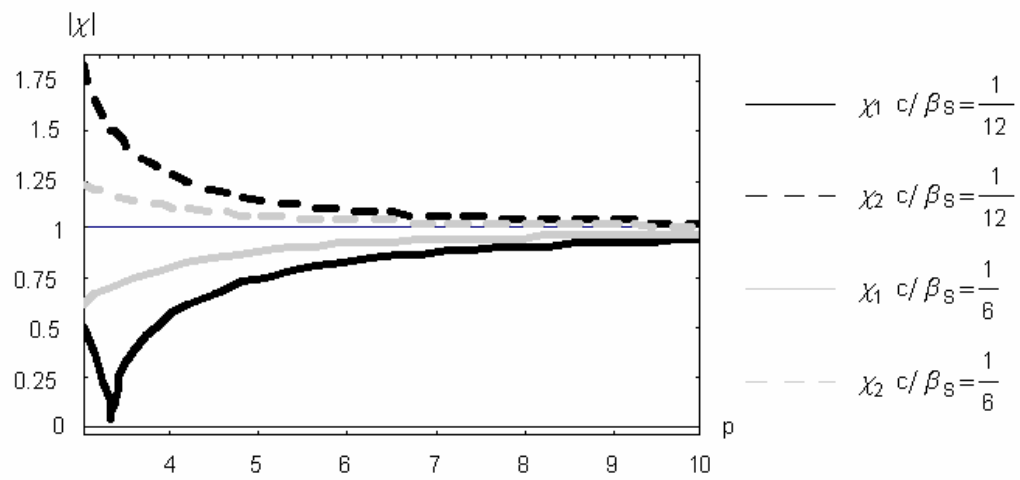

Figura 5.2 - Valor de $\chi$ para $\beta_{\mathrm{s}}=1 / 12$ e $\beta_{\mathrm{s}}=1 / 6$

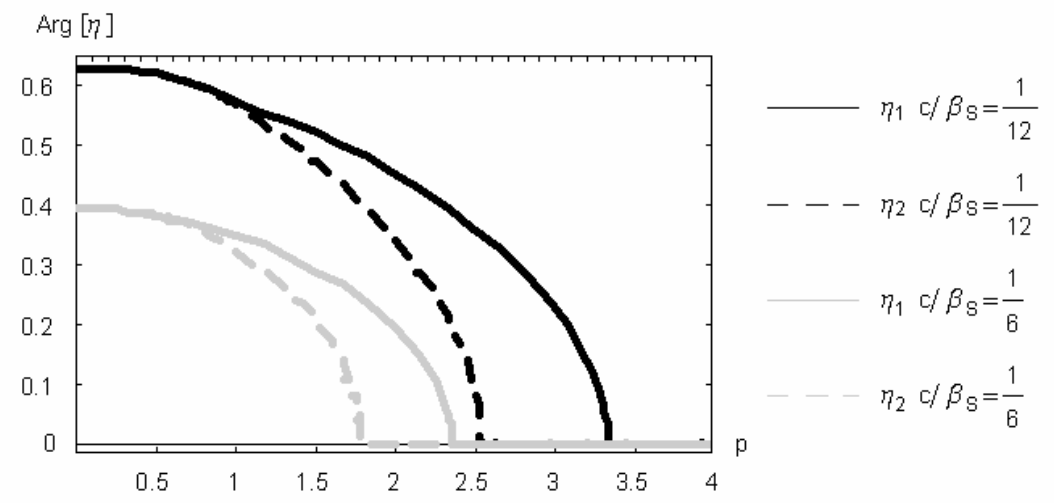

Figura 5.3 - Argumento de $\eta$ para $\beta_{\mathrm{s}}=1 / 12$ e $\beta_{\mathrm{s}}=1 / 6$.

Comparando-se a formulação consistente clássica com o modelo matricial (5.9) para $\beta_{\mathrm{s}}=1 / 12$, percebe-se que a convergência da segunda formulação é extremamente elevada para $\eta$, porém para a relação $\chi 0$ modelo consistente 
clássico tem melhor desempenho. Nota-se também que o modelo consistente é menos suscetível ao fenômeno evanescente.

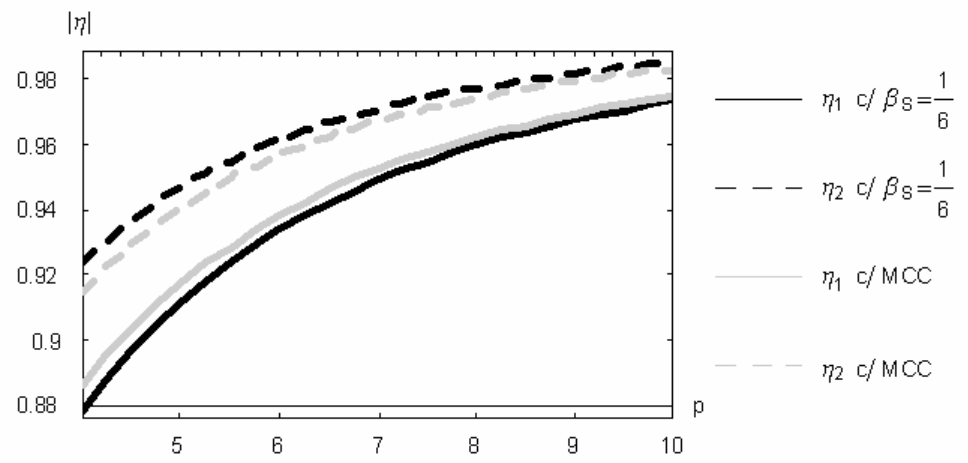

Figura 5.4 - Módulo de $\eta$ para $\beta_{\mathrm{s}}=1 / 6$ e o modelo consistente clássico.

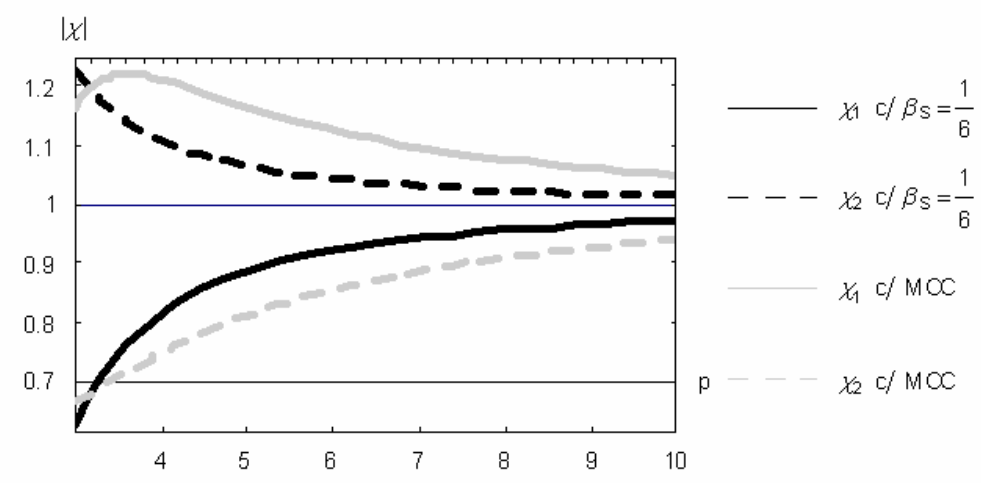

Figura 5.5 - Valor de $\chi$ para $\beta_{\mathrm{s}}=1 / 6$ e o modelo consistente clássico.

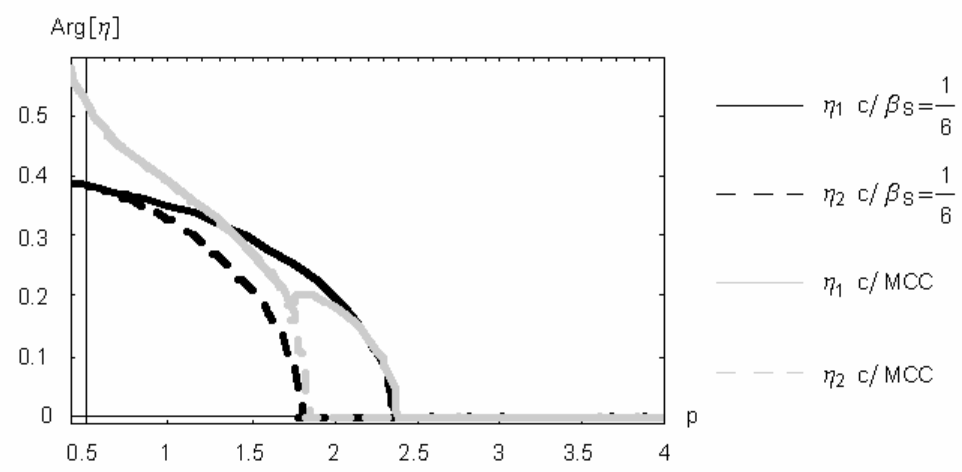

Figura 5.6 - Argumento de $\eta$ para $\beta_{\mathrm{s}}=1 / 6$ e o modelo consistente clássico.

Quanto à freqüência espacial (magnitude e ângulo de fase), a resposta dos dois casos possui erro de truncamento com magnitudes aproximadamente iguais. Entretanto, o parâmetro $\chi$ é mais próximo da unidade para o caso $\beta_{\mathrm{s}}=1 / 6$. 
Novamente, por se tratar de um integrador inspirado na quadratura numérica de Newmark, é intuitivamente interessante considerar-se uma formulação com $\beta_{\mathrm{s}}=1 / 4$. Uma última formulação a ser abordada é aquela que conduz a um menor esforço computacional conferido por $\beta_{\mathrm{s}}=0$.

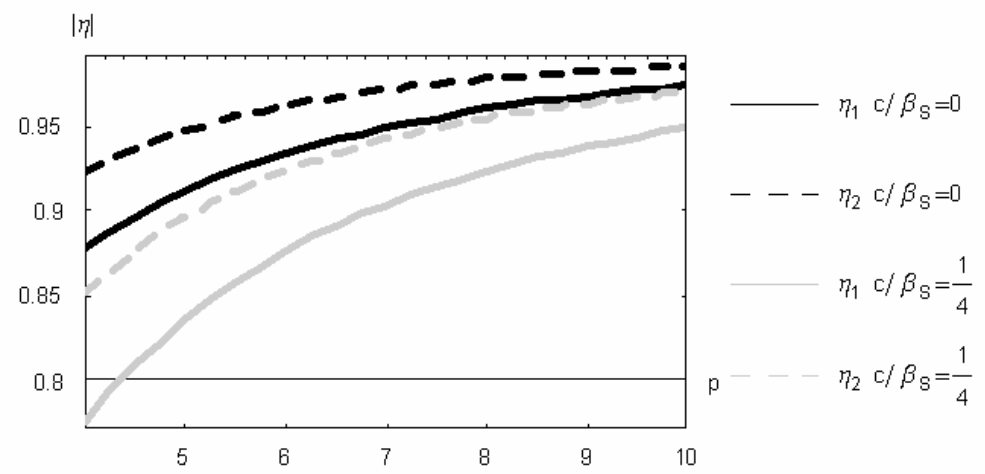

Figura 5.7 - Módulo de $\eta$ para $\beta_{\mathrm{s}}=0$ e $\beta_{\mathrm{s}}=1 / 4$.

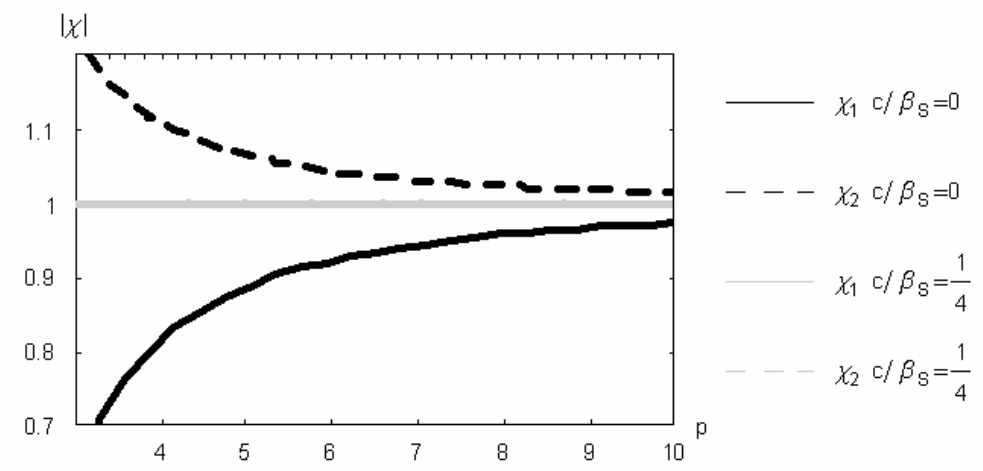

Figura $5.8-$ Valor de $\chi$ para $\beta_{\mathrm{s}}=0$ e $\beta_{\mathrm{s}}=1 / 4$

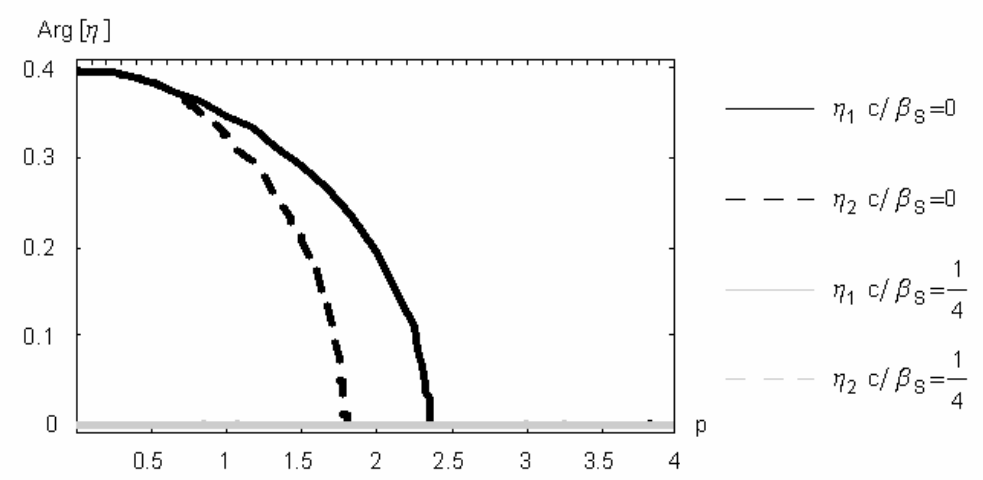

Figura 5.9 - Argumento de $\eta$ para $\beta_{\mathrm{s}}=0$ e $\beta_{\mathrm{s}}=1 / 4$.

As duas últimas figuras apontam que o modelo com $\beta_{\mathrm{s}}=1 / 4$, embora esteja associado a um erro considerável no tocante à freqüência espacial, não apresenta 
zona evanescente e tem resposta praticamente exata para $\Lambda_{\mathrm{N}}$. As tabelas seguintes retratam em números os valores plotados anteriormente para $\mathrm{p}$ igual a cinco, dez e quinze.

\begin{tabular}{|c|c|c|c|c|c|}
\hline$|\eta 1|$ & $\beta s=1 / 12$ & $\beta s=1 / 6$ & $\beta s=1 / 4$ & Consistente & $\beta s=0$ \\
\hline$p=5$ & 1.0136 & 0.9116 & 0.8360 & 0.9174 & 1.1642 \\
\hline$p=10$ & 0.9999 & 0.9731 & 0.9484 & 0.9749 & 1.0289 \\
\hline$p=15$ & 0.9997 & 0.9875 & 0.9758 & 0.9884 & 1.0123 \\
\hline
\end{tabular}

Tabela 5.1 - Valores de $\left|\eta_{1}\right|$.

\begin{tabular}{|c|c|c|c|c|c|}
\hline$\left|\eta_{2}\right|$ & $\beta \mathrm{s}=1 / 12$ & $\beta \mathrm{s}=1 / 6$ & $\beta \mathrm{s}=1 / 4$ & Consistente & $\beta \mathrm{s}=0$ \\
\hline $\mathrm{p}=5$ & 1.0078 & 0.9469 & 0.8959 & 0.9405 & 1.0826 \\
\hline $\mathrm{p}=10$ & 1.0009 & 0.9851 & 0.9699 & 0.9832 & 1.0176 \\
\hline $\mathrm{p}=15$ & 1.0003 & 0.9932 & 0.9862 & 0.9923 & 1.0076 \\
\hline
\end{tabular}

Tabela 5.2 - Valores de $\left|\eta_{2}\right|$.

\begin{tabular}{|c|c|c|c|c|c|}
\hline$\left|\chi_{1}\right|$ & $\beta \mathrm{s}=1 / 12$ & $\beta \mathrm{s}=1 / 6$ & $\beta \mathrm{s}=1 / 4$ & Consistente & $\beta \mathrm{s}=0$ \\
\hline $\mathrm{p}=5$ & 0.7542 & 0.8871 & 1.0000 & 1.1663 & 0.5875 \\
\hline $\mathrm{p}=10$ & 0.9456 & 0.9733 & 1.0000 & 1.0512 & 0.9169 \\
\hline $\mathrm{p}=15$ & 0.9763 & 0.9882 & 1.0000 & 1.0235 & 0.9641 \\
\hline
\end{tabular}

Tabela 5.3 - Valores de $\left|\chi_{1}\right|$.

\begin{tabular}{|c|c|c|c|c|c|}
\hline$\left|\chi_{2}\right|$ & $\beta \mathrm{s}=1 / 12$ & $\beta \mathrm{s}=1 / 6$ & $\beta \mathrm{s}=1 / 4$ & Consistente & $\beta \mathrm{s}=0$ \\
\hline $\mathrm{p}=5$ & 1.1480 & 1.0655 & 1.0000 & 0.8119 & 1.2561 \\
\hline $\mathrm{p}=10$ & 1.0310 & 1.0151 & 1.0000 & 0.9403 & 1.0478 \\
\hline $\mathrm{p}=15$ & 1.0134 & 1.0066 & 1.0000 & 0.9721 & 1.0203 \\
\hline
\end{tabular}

Tabela 5.4 - Valores de $\left|\chi_{2}\right|$.

\begin{tabular}{|c|c|c|c|c|c|}
\hline $\operatorname{Arg}\left[\eta_{1}\right]$ & $\beta s=1 / 12$ & $\beta s=1 / 6$ & $\beta s=1 / 4$ & Consistente & $\beta s=0$ \\
\hline$p=1$ & 0.5742 & -0.3489 & 0.0000 & 0.3869 & 0.9217 \\
\hline$p=2$ & 0.4550 & -0.1963 & 0.0000 & 0.1833 & 0.6914 \\
\hline$p=3$ & 0.2304 & 0.0000 & 0.0000 & 0.0000 & 0.4776 \\
\hline
\end{tabular}

Tabela 5.5 - Valores de $\operatorname{Arg}\left[\eta_{1}\right]$.

\begin{tabular}{|c|c|c|c|c|c|}
\hline $\operatorname{Arg}\left[\eta_{2}\right]$ & $\beta s=1 / 12$ & $\beta s=1 / 6$ & $\beta s=1 / 4$ & Consistente & $\beta s=0$ \\
\hline$p=1$ & 0.5742 & 0.3259 & 0.0000 & 0.3869 & 0.9217 \\
\hline$p=2$ & 0.3446 & 0.0000 & 0.0000 & 0.0000 & 0.5829 \\
\hline$p=3$ & 0.0000 & 0.0000 & 0.0000 & 0.0000 & 0.1780 \\
\hline
\end{tabular}

Tabela 5.6 - Valores de $\operatorname{Arg}\left[\eta_{2}\right]$.

Seja agora considerado que a discretização espacial se mantenha fixa, com $p=5$ e $\beta_{\mathrm{s}}=1 / 12$. Visando-se, pois, verificar a influência do integrador temporal, 
traçam-se as curvas de $\eta$ e $\chi$ em função de $\xi$ para $\beta=1 / 12$ e $\beta=1 / 4$, conforme ilustrado nas figuras 5.10 e 5.11 .

Naturalmente, o formato da curva está relacionado com a quadratura numérica temporal, enquanto que seu cruzamento no eixo das ordenadas depende da integração espacial.

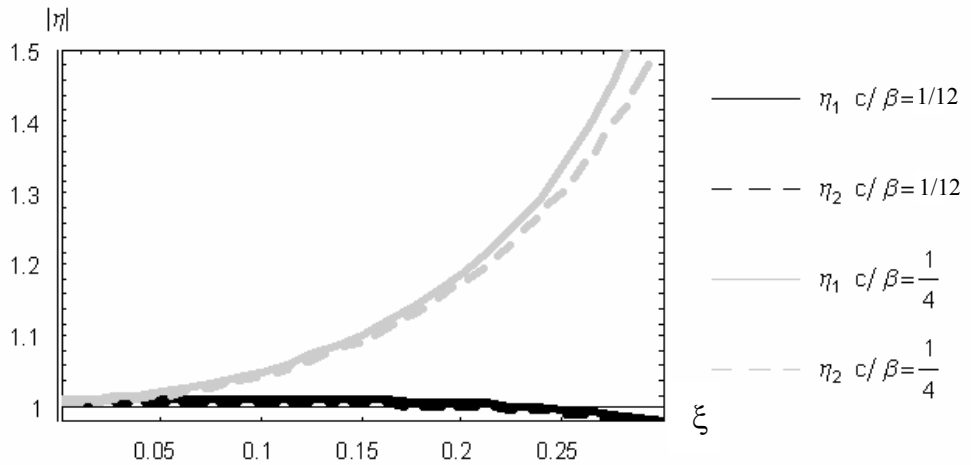

Figura $5.10-$ Valor de $\eta$ para $\beta=1 / 12$ e $\beta=1 / 4$

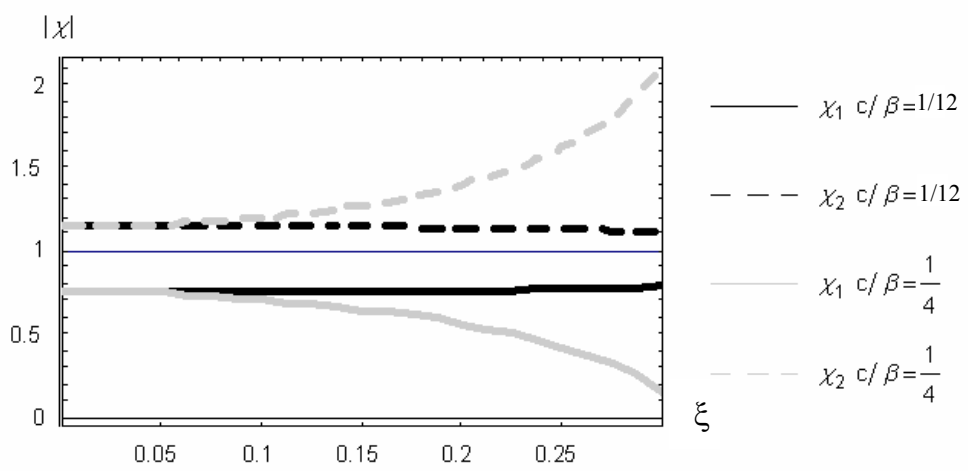

Figura 5.11 - Valor de $\chi$ para $\beta=1 / 12$ e $\beta=1 / 4$

Resta agora examinar o confronto de resultados das matrizes advindas da formulação generalizada. As figuras seguintes comparam inicialmente 0 comportamento das matrizes $\left[\mathrm{M}_{\mathrm{Y}}\right]$ e $\left[\mathrm{K}_{\mathrm{Y}}\right]$ de (5.27) com as matrizes redigidas em (5.9), para $\beta_{\mathrm{s}}=1 / 6$. 


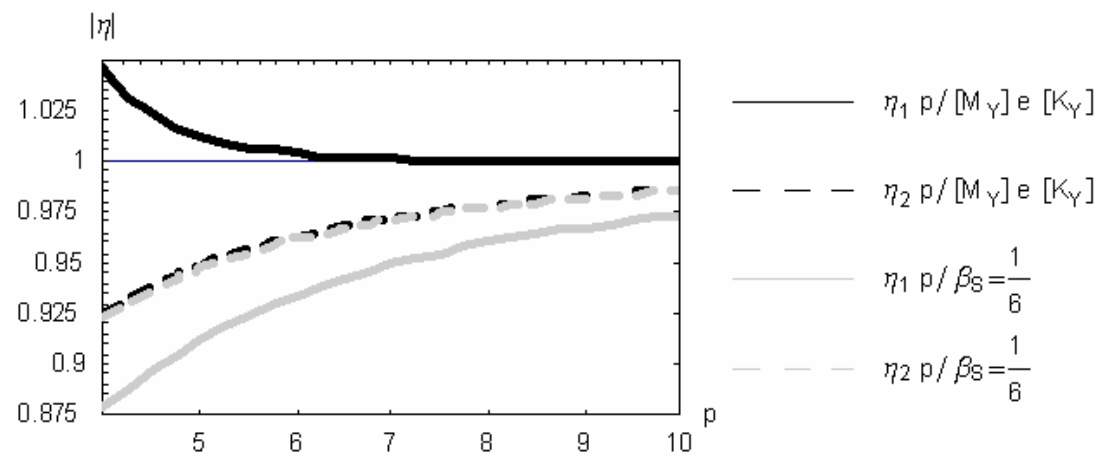

Figura 5.12 - Módulo de $\eta$ para a formulação generalizada $Y$ e $\beta_{\mathrm{s}}=1 / 6$.

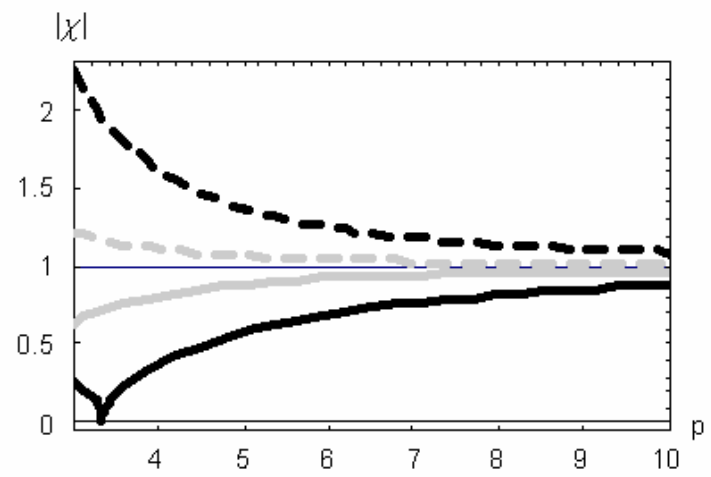

$\longrightarrow \chi_{1} p /\left[\mathrm{M}_{Y}\right] \mathrm{e}\left[\mathrm{K}_{Y}\right]$

$--\chi_{2} p /\left[\mathrm{M}_{Y}\right] \mathrm{e}\left[\mathrm{K}_{Y}\right]$

$\chi_{1} c / \beta_{\mathrm{s}}=\frac{1}{6}$

$\chi_{2} c / \beta_{\mathrm{S}}=\frac{1}{6}$

Figura 5.13 - Valor de $\chi$ para a formulação generalizada $Y$ e $\beta_{\mathrm{s}}=1 / 6$.

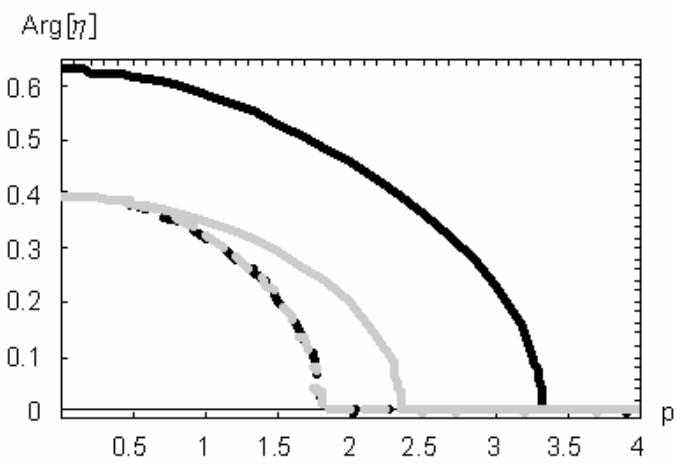

$-\eta_{1} \mathrm{p} /\left[\mathrm{M}_{\mathrm{Y}}\right] \mathrm{e}\left[\mathrm{K}_{\mathrm{Y}}\right]$

$--r_{2} p /\left[\mathrm{M}_{\mathrm{Y}}\right] \mathrm{e}\left[\mathrm{K}_{\mathrm{Y}}\right]$

$\eta_{1} \mathrm{p} / \beta_{\mathrm{S}}=\frac{1}{6}$

$\eta_{2} \mathrm{p} / \beta_{\mathrm{S}}=\frac{1}{6}$

Figura 5.14 - Argumento de $\eta$ para a formulação generalizada $Y$ e $\beta_{\mathrm{s}}=1 / 6$.

A formulação generalizada $Y$ apresenta melhor desempenho apenas quanto ao parâmetro $\eta_{1}$. Nos demais aspectos, a formulação com $\beta_{\mathrm{s}}=1 / 6$ se mostra vantajosa. Vale apontar que o valor de $\eta_{2}$ é praticamente igual para ambos os casos. Comparando-se agora a formulação generalizada $\Theta$ com $\beta_{\mathrm{s}}=1 / 12$, vê-se que a diferença numérica entre os módulo de $\eta$ para a segunda raiz é desprezível. 


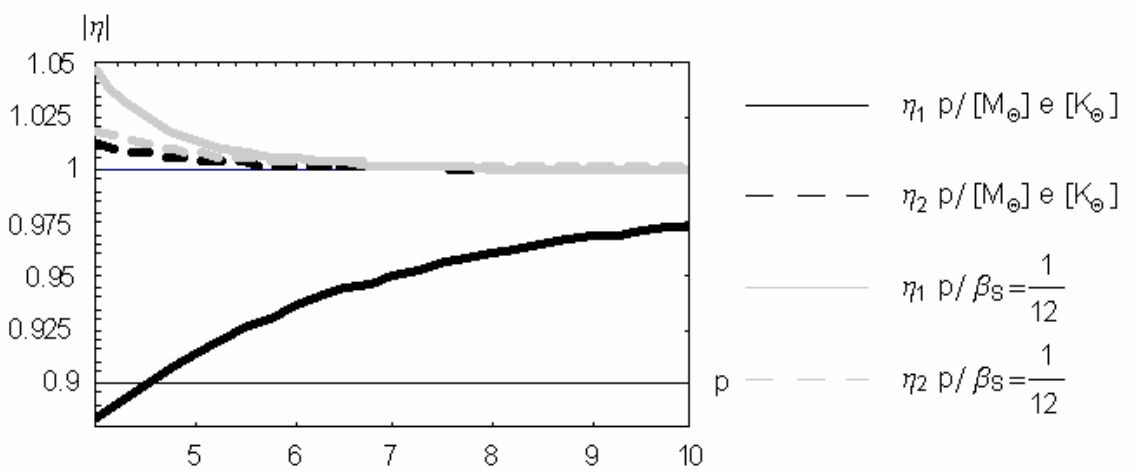

Figura 5.15 - Módulo de $\eta$ para a formulação generalizada $\Theta$ e $\beta_{\mathrm{s}}=1 / 12$.

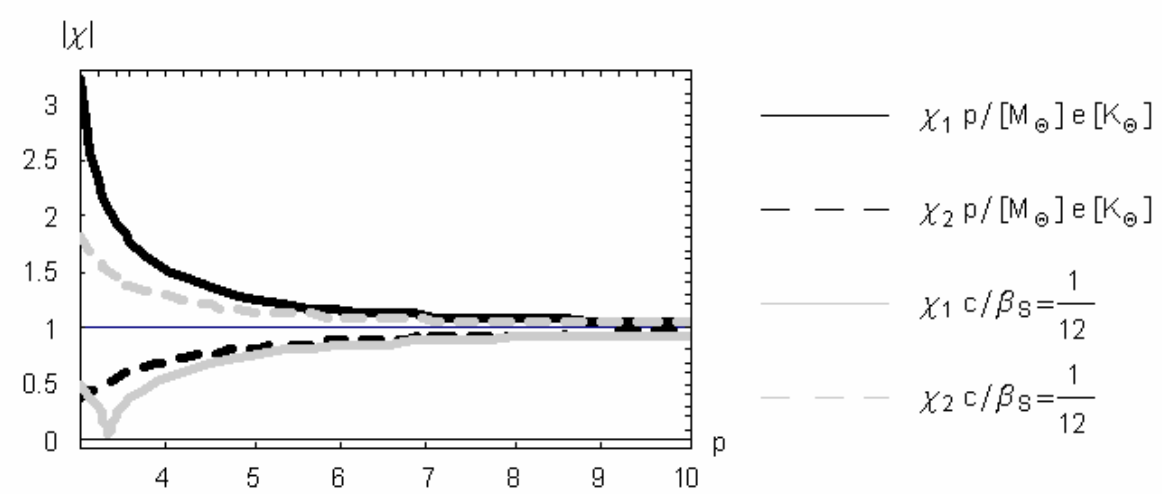

Figura 5.16 - Valor de $\chi$ para a formulação generalizada $\Theta$ e $\beta_{\mathrm{s}}=1 / 12$.

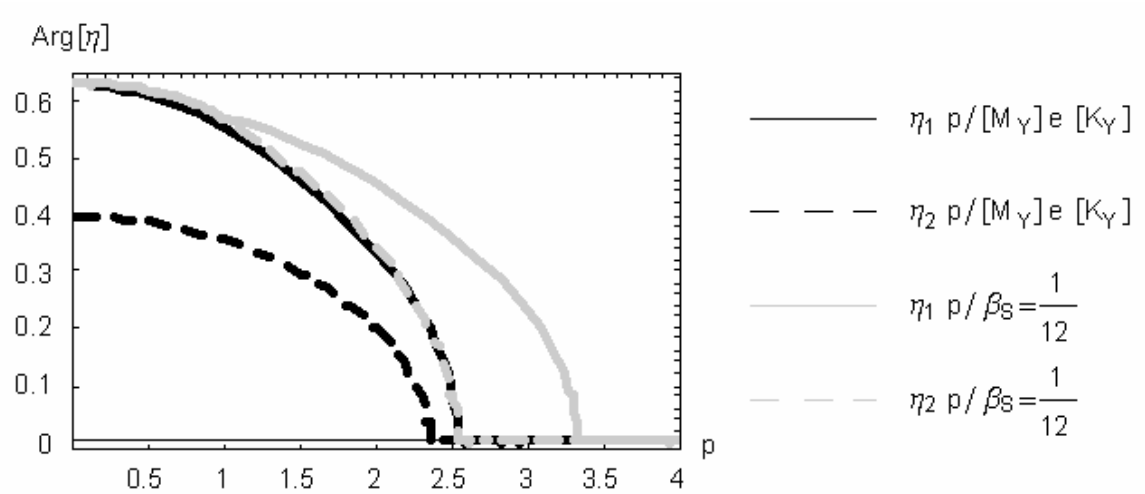

Figura 5.17 - Argumento de $\eta$ para a formulação generalizada $\Theta$ e $\beta_{\mathrm{s}}=1 / 12$.

A fim de verificar o comportamento das formulações desenvolvidas perante o efeito das ondas espúrias, as figuras seguintes ilustram os valores do módulo $|\kappa / l|$ para a formulação com matriz consistente clássica (MCC) e com $\beta_{S}=1 / 6$, $\beta_{S}=1 / 4$ e $\beta_{S}=1 / 12$, fixando-se $p_{1}=5, Z=1, \gamma=1.3, \xi=0.02$ e $\beta=1 / 4$. 


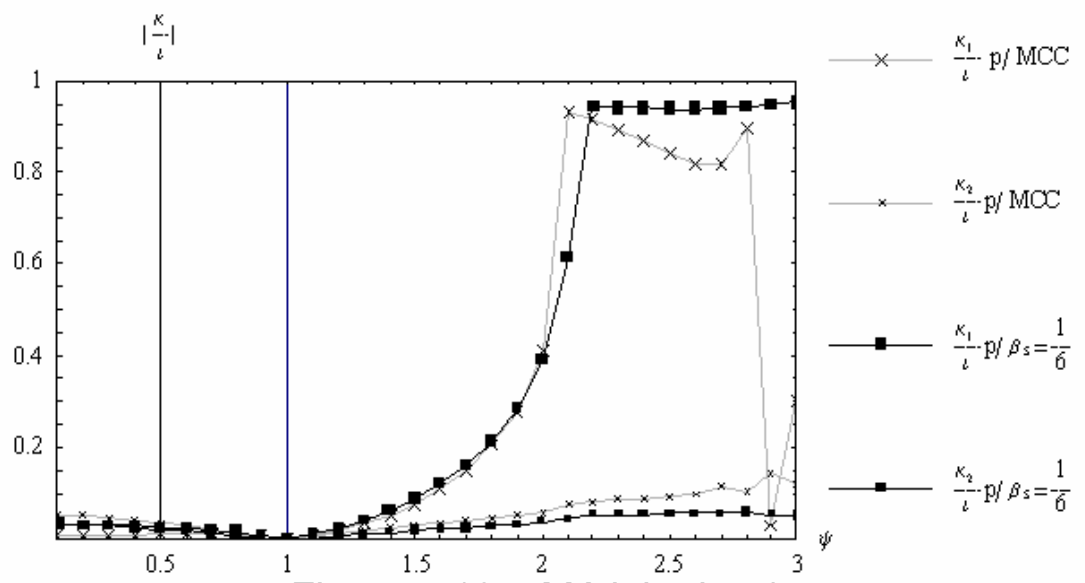

Figura 5.18 - Módulo de $\kappa /$.

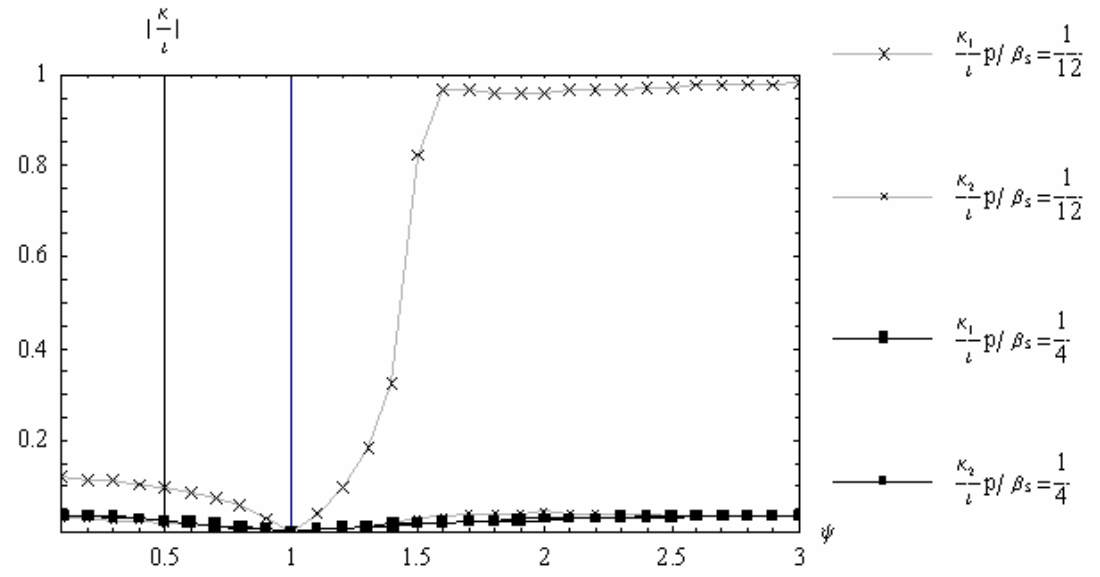

Figura 5.19 - Módulo de $\kappa / 1$.

Pela figura 5.18, pode-se observar que a matriz de massa consistente apresenta, de fato, valores das amplitudes ondas envolvidas semelhantes aos encontrados para o caso do integrador $\beta_{S} \operatorname{com} \beta_{S}=1 / 6$.

Para $\beta_{S}=1 / 4$, percebe-se considerável redução do fenômeno espúrio. Já para $\beta_{S}=1 / 12$, o efeito espúrio é significativo. De forma geral, pode-se concluir que tal fenômeno de natureza numérica torna-se mais crítico conforme o valor de $\beta_{S}$ decresce.

A formulação que emprega o intagrador $\beta_{S}$ demonstra ainda uma excelente resposta em relação à segunda freqüência espacial, apresentando praticamente reflexão espúria apenas na primeira freqüência espacial. Desta maneira, observase que seu comportamento é muito parecido com o caso do elemento de barra discutido anteriormente. 


\section{$\underline{\text { Capítulo } 6}$}

\section{Considerações Teóricas Complementares para a Elaboração de Algoritmos de Integração para Elementos Finitos em Análises Dinâmicas}

\subsection{INTRODUCÃO}

O emprego de funções aproximadoras polinomiais envolvendo valores nodais da função e de suas derivadas formando-se assim uma combinação de parâmetros incógnitos em número discreto, resultando finalmente numa equação de diferença com solução não finita em um formato exponencial para o campo de deslocamentos (incluindo os giros, naturalmente), é novamente a base da formulação de elementos finitos apresentada neste capítulo. Como sabido, tal aproximação gera um erro local de truncamento que, apesar de teoricamente ser um polinômio de grau infinito, os termos de menor potência são nulos, sendo a 
ordem de convergência governada pelo termo de menor potência não nulo no desenvolvimento, também conhecido na literatura como ordem do erro.

Uma vez que as acelerações $\ddot{\theta}$ e ÿ participam acopladamente na equação diferencial de equilíbrio de forças e de momentos fletores respectivamente, propõe-se, em um primeiro enfoque, acoplar as equações diferencias de forma que as parcelas de erro se anulem no máximo grau possível. Tal procedimento implica, em princípio, na obtenção de matrizes de massa ditas cheias.

\subsection{ELEVACCÃO DA ORDEM DE ERRO DOS RESÍDUOS EM ELEMENTOS DE VIGA}

Segundo a teoria de viga de Timoshenko, as equações diferenciais regentes do movimento ondulatório permitem declarar as acelerações como sendo equações diferenciais no domínio do espaço, com a redação:

$$
\begin{aligned}
& \ddot{y}=c_{S}^{2}\left(y^{\prime \prime}-\theta^{\prime}\right) \\
& \ddot{\theta}=c_{R}^{2} \theta^{\prime}+\frac{c_{S}^{2}}{r^{2}}\left(y^{\prime}-\theta\right)
\end{aligned}
$$

No caso do sistema de referência da figura 4.1, as equações constitutivas de momento fletor e da força cisalhante, denotadas por $\mathrm{M}$ e $\mathrm{V}$ respectivamente, em um elemento infinitesimal de viga são:

$$
\begin{aligned}
& \mathrm{M}=-\mathrm{EI} \theta^{\prime} \\
& \mathrm{V}=\mathrm{kGA}\left(\mathrm{y}^{\prime}-\theta\right)
\end{aligned}
$$

Com isso, seguindo o sistema de referência e as numerações conforme a figura 4.2 , os momentos fletores e as forças cortantes nas extremidades de um elemento finito de viga podem ser descritos como:

$$
M_{1}=-E I \theta_{1}^{\mid}
$$




$$
\begin{aligned}
& \mathrm{M}_{2}=\mathrm{EI} \theta_{2}^{\prime} \\
& \mathrm{V}_{1}=-\mathrm{kGA}\left(\mathrm{y}_{1}^{\prime}-\theta_{1}\right) \\
& \mathrm{V}_{2}=\mathrm{kGA}\left(\mathrm{y}_{2}^{\prime}-\theta_{2}\right)
\end{aligned}
$$

Observando tais princípios físicos, que consideram termos discretos, pretende-se aplicar o equilíbrio de ações em um único elemento finito. Conforme formulação clássica já bem elaborada no tocante à matriz de rigidez, é permitindo escrever-se:

$\frac{E I}{L^{3}(1+\Phi)}\left[\begin{array}{cccc}12 & 6 L & -12 & 6 L \\ 6 L & L^{2}(4+\Phi) & -6 L & L^{2}(2-\Phi) \\ -12 & -6 L & 12 & -6 L \\ k_{4} & L^{2}(2-\Phi) & -6 L & L^{2}(4+\Phi)\end{array}\right]\left[\begin{array}{c}y_{1} \\ \theta_{1} \\ y_{2} \\ \theta_{2}\end{array}\right\}+\left[\begin{array}{cccc}m_{1} & m_{2} & m_{3} & m_{4} \\ m_{2} & m_{5} & m_{6} & m_{7} \\ m_{3} & m_{6} & m_{1} & -m_{2} \\ m_{4} & m_{7} & -m_{2} & m_{5}\end{array}\right]\left[\begin{array}{c}\ddot{y}_{1} \\ \ddot{\theta}_{1} \\ \ddot{y}_{2} \\ \ddot{\theta}_{2}\end{array}\right\}-\left\{\begin{array}{c}V_{1} \\ M_{1} \\ V_{2} \\ M_{2}\end{array}\right\}=\left\{\begin{array}{c}0 \\ 0 \\ 0 \\ 0\end{array}\right\}+\left\{\begin{array}{c}R_{1} \\ R_{2} \\ R_{3} \\ R_{4}\end{array}\right\}$

onde a segunda parcela do segundo membro é o vetor que contém os resíduos numéricos, também conhecidos como erros de truncamento locais. Tais resíduos têm uma ordem de erro associada a cada linha do sistema matricial, proveniente das expansões em série de Taylor do deslocamento transversal e rotação segundo a seguinte notação:

$$
\begin{array}{ll}
y_{2}=y_{1}+y_{1}^{\prime} L+y_{1}^{\|} \frac{L^{2}}{2}+\cdots+\frac{\partial^{k} y_{1}}{\partial x^{k}} \frac{L^{k}}{k !} & (k \rightarrow \infty) \\
\theta_{2}=\theta_{1}+\theta_{1}^{l} L+\theta_{1}^{\| !} \frac{L^{2}}{2}+\cdots+\frac{\partial^{k} \theta_{1}}{\partial x^{k}} \frac{L^{k}}{k !} & (k \rightarrow \infty)
\end{array}
$$

Substituindo as igualdades (6.5), (6.3) e (6.1) na equação matricial (6.4), é possível extrair-se os valores dos resíduos em função dos parâmetros $m_{j}(j=1$ a 7 ) constantes da matriz de massa. Trata-se de uma combinação de termos discretos, onde as variáveis são $m_{j}(j=1$ a 7$)$. Este ponto de vista é um tanto conservador uma vez que por hora não se cogita nenhuma alteração na matriz de rigidez clássica.

Efetuando-se os cálculos de forma a isolar os coeficientes dos termos discretos e suas derivadas, ou seja: 


$$
R_{j}=\frac{12 E I}{L^{2}(1+\Phi)}\left\{\begin{array}{c}
y_{1} C_{1, j}+y_{1}^{\prime} C_{2, j}+y_{1}^{\| \prime} C_{3, j}+y_{1}^{\| \prime} C_{4, j} \\
\theta_{1} D_{1, j}+\theta_{1}^{\mid} D_{2, j}+\theta_{1}^{\prime \prime} D_{3, j}+\theta_{1}^{\| \prime \prime} D_{4, j}
\end{array}\right\} \quad(j=1 a 4)
$$

onde:

$$
\begin{aligned}
& \mathrm{C}_{1, \mathrm{j}}=0 \\
& (j=1 \text { a } 4) \\
& D_{1,1}=-C_{2,1}=-\frac{1}{\Phi}-\frac{1+\Phi}{m r^{2} \Phi}\left(m_{2}+m_{4}\right) \\
& \mathrm{D}_{1,2}=\mathrm{D}_{1,4}=-\mathrm{C}_{2,2}=-\mathrm{C}_{2,4}=\frac{\mathrm{L}}{2}-\frac{1+\Phi}{\mathrm{mr}^{2} \Phi}\left(\mathrm{m}_{5}+\mathrm{m}_{7}\right) \\
& \mathrm{D}_{1,3}=\mathrm{C}_{2,3}=-\frac{1}{\Phi}+\frac{1+\Phi}{\mathrm{mr}^{2} \Phi}\left(\mathrm{m}_{6}-\mathrm{m}_{2}\right) \\
& \mathrm{D}_{2,1}=-\mathrm{C}_{3,1}=\frac{\mathrm{L}}{2}-\frac{1+\Phi}{\mathrm{m} \Phi}\left(\mathrm{m}_{1}+\mathrm{m}_{3}+\frac{\mathrm{L}}{\mathrm{r}^{2}} \mathrm{~m}_{4}\right) \\
& D_{2,2}=-C_{3,2}=\frac{L^{2}}{4}-\frac{1+\Phi}{m \Phi}\left(m_{2}+m_{6}+\frac{L}{r^{2}} m_{7}\right) \\
& \mathrm{D}_{2,3}=-\mathrm{C}_{3,3}=\frac{2+\Phi}{2 \Phi} \mathrm{L}-\frac{1+\Phi}{\mathrm{m} \Phi}\left(\mathrm{m}_{1}+\mathrm{m}_{3}-\frac{\mathrm{L}}{\mathrm{r}^{2}} \mathrm{~m}_{2}\right) \\
& \mathrm{D}_{2,4}=-\mathrm{C}_{3,4}=\frac{\mathrm{L}^{2}}{4}-\frac{1+\Phi}{\mathrm{m} \Phi}\left(\mathrm{m}_{4}-\mathrm{m}_{2}+\frac{\mathrm{L}}{\mathrm{r}^{2}} \mathrm{~m}_{5}\right) \\
& D_{3,1}=\frac{L^{2}}{4}-\frac{1+\Phi}{m \Phi} L m_{3}+\frac{L^{2}}{12 m r^{2} \Phi}\left(\Phi^{2}-5 \Phi-6\right) m_{4}+\frac{1+\Phi}{12 m r^{2}} L^{2} m_{2} \\
& D_{3,2}=\frac{2-\Phi}{24} L^{3}-\frac{1+\Phi}{m \Phi} L m_{6}+\frac{L^{2}}{12 m r^{2} \Phi}\left(\Phi^{2}-5 \Phi-6\right) m_{7}+\frac{1+\Phi}{12 m r^{2}} L^{2} m_{5} \\
& D_{3,3}=\frac{2+\Phi}{4 \Phi} L-\frac{1+\Phi}{4 \Phi} L m_{1}-\frac{L^{2}}{12 m r^{2} \Phi}\left(\Phi^{2}-5 \Phi-6\right) m_{2}+\frac{1+\Phi}{12 m r^{2}} L^{2} m_{6} \\
& D_{3,4}=\frac{2-\Phi}{24} L^{3}+\frac{1+\Phi}{m \Phi} L m_{2}+\frac{L^{2}}{12 m r^{2} \Phi}\left(\Phi^{2}-5 \Phi-6\right) m_{5}+\frac{1+\Phi}{12 m r^{2}} L^{2} m_{7}
\end{aligned}
$$




$$
\begin{aligned}
& \mathrm{C}_{4,1}=-\frac{\mathrm{L}^{2}}{6}+\frac{1+\Phi}{\mathrm{m} \Phi} \mathrm{L}\left(\mathrm{m}_{3}+\frac{\mathrm{L}}{2 \mathrm{r}^{2}} \mathrm{~m}_{4}\right) \\
& C_{4,2}=-\frac{L^{3}}{12}+\frac{1+\Phi}{m \Phi} L\left(m_{6}+\frac{L}{2 r^{2}} m_{7}\right) \\
& \mathrm{C}_{4,3}=-\frac{3+2 \Phi}{6 \Phi} \mathrm{L}^{2}+\frac{1+\Phi}{\mathrm{m} \Phi} \mathrm{L}\left(\mathrm{m}_{1}-\frac{\mathrm{L}}{2 \mathrm{r}^{2}} \mathrm{~m}_{2}\right) \\
& C_{4,4}=-\frac{L^{2}}{12}+\frac{1+\Phi}{m \Phi} L\left(\frac{L}{2 r^{2}} m_{5}-m_{2}\right) \\
& \mathrm{D}_{4,1}=\frac{\mathrm{L}^{3}}{12}-\frac{1+\Phi}{2 m \Phi} \mathrm{L}^{2} \mathrm{~m}_{3}+\frac{\mathrm{L}^{3}}{12 m r^{2} \Phi}\left(\Phi^{2}-\Phi-2\right) \mathrm{m}_{4} \\
& D_{4,2}=\frac{2-\Phi}{72} L^{4}-\frac{1+\Phi}{2 m \Phi} L^{2} m_{6}+\frac{L^{3}}{12 m r^{2} \Phi}\left(\Phi^{2}-\Phi-2\right) m_{7} \\
& D_{4,3}=\frac{2+\Phi}{12 \Phi} L^{3}-\frac{1+\Phi}{2 m \Phi} L^{2} m_{1}-\frac{L^{3}}{12 m r^{2} \Phi}\left(\Phi^{2}-\Phi-2\right) m_{2} \\
& D_{4,4}=\frac{1-2 \Phi}{72} L^{4}-\frac{1+\Phi}{2 m \Phi} L^{2} m_{2}+\frac{L^{3}}{12 m r^{2} \Phi}\left(\Phi^{2}-\Phi-2\right) m_{5} \\
& C_{5,1}=-\frac{L^{3}}{24}+\frac{1+\Phi}{2 m \Phi} L^{2}\left(m_{3}+\frac{L}{3 r^{2}} m_{4}\right) \\
& C_{5,2}=-\frac{L^{4}}{48}+\frac{1+\Phi}{2 m \Phi} L^{2}\left(m_{6}+\frac{L}{3 r^{2}} m_{7}\right) \\
& C_{5,3}=-\frac{4+3 \Phi}{24 \Phi} L^{3}+\frac{1+\Phi}{2 m \Phi} L^{2}\left(m_{1}-\frac{L}{3 r^{2}} m_{2}\right) \\
& C_{5,4}=-\frac{L^{4}}{48}+\frac{1+\Phi}{2 m \Phi} L^{2}\left(\frac{L}{3 r^{2}} m_{5}-m_{2}\right)
\end{aligned}
$$

Considerando-se que o parâmetro $\Phi$ tem ordem $\mathrm{L}^{-2}$, é interessante retirar do sistema esta dependência, implícita, do comprimento $L$, fazendo-se a substituição: 


$$
\Phi=\frac{\phi}{L^{2}}
$$

Desenvolvendo o sistema de equações, conclui-se que para os valores:

$$
\begin{array}{lll}
\mathrm{m}_{1}=\frac{\Phi}{1+\Phi} \frac{\rho \mathrm{AL}}{3} & ; & \mathrm{m}_{2}=-\frac{\rho A r^{2}}{1+\Phi} \\
\mathrm{m}_{3}=\frac{\Phi}{1+\Phi} \frac{\rho \mathrm{AL}}{6} & ; & \mathrm{m}_{4}=\mathrm{m}_{6}=0 \\
\mathrm{~m}_{5}=\frac{\rho \mathrm{A}\left(\Phi \mathrm{L}^{2} \mathrm{r}^{2}-4 \mathrm{r}^{4}\right)}{4 \mathrm{~L}(1+\Phi)} \quad ; & \mathrm{m}_{7}=\frac{\rho \mathrm{A}\left(\Phi \mathrm{L}^{2} \mathrm{r}^{2}+4 \mathrm{r}^{4}\right)}{4 \mathrm{~L}(1+\Phi)}
\end{array}
$$

os componentes do vetor de resíduos são das seguintes ordens de erro em L:

$$
\begin{array}{lll}
\mathrm{R}_{1}=\mathrm{O}\left[\mathrm{L}^{3}\right] & ; & \mathrm{R}_{2}=\mathrm{O}\left[\mathrm{L}^{2}\right] \\
\mathrm{R}_{3}=\mathrm{O}\left[\mathrm{L}^{3}\right] & ; & \mathrm{R}_{4}=\mathrm{O}\left[\mathrm{L}^{2}\right]
\end{array}
$$

\subsection{EQUILÍBRIO DE FORCCAS TRANSVERSAIS EM ELEMENTOS DE VIGA}

Reescrevendo-se as equações diferenciais de equilíbrio de forças e de momentos fletores como um sistema acoplado unidimensional discretizado, temse:

$$
\begin{aligned}
& \ddot{y}_{n, j}-c_{s}^{2} y_{n, j}^{\prime \prime}+c_{s}^{2} \theta_{n, j}^{\prime}=0 \\
& r^{2} \ddot{\theta}_{n, j}-c_{R}^{2} r^{2} \theta_{n, j}^{\prime \prime}-c_{s}^{2} y_{n, j}^{\prime}+c_{s}^{2} \theta_{n, j}=0
\end{aligned}
$$

onde $\mathrm{n}$ e j representam o passo de tempo e o nó respectivamente.

Derivando a equação (6.11b) e substituindo-a em (6.11a) chega-se à equação acoplada:

$$
\ddot{y}_{n, j}-r^{2} \ddot{\theta}_{n, j}^{\prime}+c_{R}^{2} r^{2} \theta_{n, j}^{\prime \prime \prime}=0
$$


A meta é elaborar uma única equação de equilíbrio de forças de D’Alembert, resultante de uma combinação de (6.11a) e (6.12). As soluções analíticas de cada uma, no domínio do espaço, são:

$$
\begin{aligned}
& \ddot{y}(x, t)+\alpha^{2} c_{s}^{2} y(x, t)+i \alpha c_{s}^{2} \theta(x, t)=0 \\
& \ddot{y}(x, t)-i \alpha r^{2} \ddot{\theta}(x, t)+(i \alpha)^{3} c_{R}^{2} r^{2} \theta(x, t)=0
\end{aligned}
$$

Admitindo-se o formato numérico do campo de deslocamentos conforme descrito na expressão (4.19), procura-se uma combinação discreta de tal sorte que a expansão em série dos exponenciais (que se tornam funções trigonométricas do tipo seno e cosseno) retorne, a menos de um erro de determinada ordem, à equação de movimento dos deslocamentos transversais.

Para o caso da viga infinita composta de elementos simples, onde ocorre a contribuição de três nós no equilíbrio de forças, a aproximação numérica das derivadas pode ser auxiliada pelo resumo das operações algébricas:

$$
\begin{aligned}
& y_{n, j}^{\prime}=\left(a_{1} e^{-i \alpha_{N} L}+2 a_{2}+a_{3} e^{i \alpha_{N} L}\right) y_{n, j}=\nabla^{a} y_{n, j} \\
& y_{n, j}^{\prime \prime}=\left(b_{1} e^{-i \alpha_{N} L}+2 b_{2}+b_{3} e^{i \alpha_{N} L}\right) y_{n, j}=\stackrel{b}{\nabla} y_{n, j} \\
& \theta_{n, j}^{\prime}=\left(A_{1} e^{-i \alpha_{N} L}+2 A_{2}+A_{3} e^{i \alpha_{N} L}\right) \theta_{n, j}=\stackrel{A}{\nabla} \theta_{n, j} \\
& \theta_{n, j}^{\prime \prime}=\left(B_{1} e^{-i \alpha_{N} L}+2 B_{2}+B_{3} e^{i \alpha_{N} L}\right) \theta_{n, j}=\stackrel{B}{\nabla} \theta_{n, j} \\
& \ddot{\theta}_{n, j}^{\prime}=\left(C_{1} e^{-i \alpha_{N} L}+2 C_{2}+C_{3} e^{i \alpha_{N} L}\right) \ddot{\theta}_{n, j}=\stackrel{C}{\nabla} \ddot{\theta}_{n, j} \\
& \theta_{n, j}^{\prime \prime \prime}=\left(D_{1} e^{-i \alpha_{N} L}+2 D_{2}+D_{3} e^{i \alpha_{N} L}\right) \theta_{n, j}=\stackrel{D}{\nabla} \theta_{n, j}
\end{aligned}
$$

A substituição direta destas igualdades em (6.11a) e (6.12), juntamente com uma combinação independente dos termos que multiplicam a aceleração ÿ para cada equação, conduz a: 


$$
\begin{aligned}
& \ddot{y}_{n, j}-c_{S}^{2} \frac{b_{1} e^{-i \alpha_{N} L}+2 b_{2}+b_{3} e^{i \alpha_{N} L}}{m_{1} e^{-i \alpha_{N} L}+2 m_{2}+m_{3} e^{i \alpha_{N} L}} y_{n, j}+c_{S}^{2} \frac{A_{1} e^{-i \alpha_{N} L}+2 A_{2}+A_{3} e^{i \alpha_{N} L}}{m_{1} e^{-i \alpha_{N} L}+2 m_{2}+m_{3} e^{i \alpha_{N} L}} \theta_{n, j}=0 \\
& \ddot{y}_{n, j}-r^{2} \frac{C_{1} e^{-i \alpha_{N} L}+2 C_{2}+C_{3} e^{i \alpha_{N} L}}{M_{1} e^{-i \alpha_{N} L}+2 M_{2}+M_{3} e^{i \alpha_{N} L}} \ddot{\theta}_{n, j}+c_{R}^{2} r^{2} \frac{D_{1} e^{-i \alpha_{N} L}+2 D_{2}+D_{3} e^{i \alpha_{N} L}}{M_{1} e^{-i \alpha_{N} L}+2 M_{2}+M_{3} e^{i \alpha_{N} L}} \theta_{n, j}=0
\end{aligned}
$$

Valendo-se ressaltar que este último conjunto de equações é semelhante ao expresso em (3.12). Comparando-se (6.13) e (6.14), fica evidente que os parâmetros combinatórios devem resultar em uma expansão que obedece as seguintes tendências:

$$
\begin{aligned}
& -c_{S}^{2} \frac{b_{1} e^{-i \alpha_{N} L}+2 b_{2}+b_{3} e^{i \alpha_{N} L}}{m_{1} e^{-i \alpha_{N} L}+2 m_{2}+m_{3} e^{i \alpha_{N} L}} \rightarrow \alpha^{2} c_{S}^{2} \\
& c_{S}^{2} \frac{A_{1} e^{-i \alpha_{N} L}+2 A_{2}+A_{3} e^{i \alpha_{N} L}}{m_{1} e^{-i \alpha_{N} L}+2 m_{2}+m_{3} e^{i \alpha_{N} L}} \rightarrow i \alpha c_{S}^{2} \\
& -r^{2} \frac{C_{1} e^{-i \alpha_{N} L}+2 C_{2}+C_{3} e^{i \alpha_{N} L}}{M_{1} e^{-i \alpha_{N} L}+2 M_{2}+M_{3} e^{i \alpha_{N} L}} \rightarrow-i \alpha r^{2} \\
& c_{R}^{2} r^{2} \frac{D_{1} e^{-i \alpha_{N} L}+2 D_{2}+D_{3} e^{i \alpha_{N} L}}{M_{1} e^{-i \alpha_{N} L}+2 M_{2}+M_{3} e^{i \alpha_{N} L}} \rightarrow(i \alpha)^{3} c_{R}^{2} r^{2}
\end{aligned}
$$

Obviamente, verifica-se de imediato que aproximar a terceira derivada com somente três pontos de integração é uma tarefa impossível. Assim, atribui-se de antemão que os fatores de combinação $D$ sejam nulos.

As funções exponenciais contidas nas expressões (6.16) conduzem ao surgimento de funções trigonométricas, cujas expansões em série convergem para valores da resposta analítica. Para isto, obtêm-se as primeiras restrições algébricas $A_{1}=-A_{3}, A_{2}=0, b_{1}=b_{3}, C_{1}=-C_{3}, C_{2}=0, M_{1}=M_{3}$ e $m_{1}=m_{3}$, implicando-se em:

$$
\frac{\stackrel{b}{m}}{\nabla}=\frac{b_{3} \cos \left(\alpha_{N} L\right)-b_{2}}{m_{3} \cos \left(\alpha_{N} L\right)-m_{2}}
$$




$$
\begin{aligned}
& \frac{A}{\nabla}=i \frac{A_{3} \operatorname{sen}\left(\alpha_{N} L\right)}{m_{3} \cos \left(\alpha_{N} L\right)-m_{2}} \\
& \frac{\stackrel{c}{M}}{\stackrel{M}{\nabla}}=i \frac{C_{3} \operatorname{sen}\left(\alpha_{N} L\right)}{M_{3} \cos \left(\alpha_{N} L\right)-M_{2}}
\end{aligned}
$$

cujas expansões em série são:

$$
\begin{aligned}
& \frac{\stackrel{b}{m}}{\nabla}=\frac{b_{3}+b_{2}}{m_{3}+m_{2}}+\frac{\alpha_{N}^{2}\left(-b_{3} m_{2}+b_{2} m_{3}\right)}{2\left(m_{2}+m_{3}\right)^{2}} L^{2}+\frac{\alpha_{N}^{4}\left(m_{2}-5 m_{3}\right)\left(b_{3} m_{2}-b_{2} m_{3}\right)}{24\left(m_{2}+m_{3}\right)^{3}} L^{4}+O\left[L^{5}\right] \\
& \frac{\stackrel{A}{m}}{\nabla}=\frac{i \alpha_{N} A_{3}}{m_{3}+m_{2}} L-\frac{i \alpha_{N}^{3} A_{3}\left(m_{2}-2 m_{3}\right)}{6\left(m_{2}+m_{3}\right)^{2}} L^{3}+\frac{i \alpha_{N}^{5} A_{3}\left(m_{2}^{2}-13 m_{2} m_{3}+16 m_{3}^{2}\right)}{120\left(m_{2}+m_{3}\right)^{3}} L^{5}+O\left[L^{7}\right] \\
& \frac{\mathrm{D}}{\mathrm{M}}=\frac{i \alpha_{N} C_{3}}{M_{3}+M_{2}} L-\frac{i \alpha_{N}^{3} C_{3}\left(M_{2}-2 M_{3}\right)}{6\left(M_{2}+M_{3}\right)^{2}} L^{3}+\frac{i \alpha_{N}^{5} C_{3}\left(M_{2}^{2}-13 M_{2} M_{3}+16 M_{3}^{2}\right)}{120\left(M_{2}+M_{3}\right)^{3}} L^{5}+O\left[L^{7}\right]
\end{aligned}
$$

Neste ponto, é possível atribuir valores para os parâmetros de combinação visando obedecer às equações diferenciais (6.13). Por exemplo, quando:

$$
\begin{array}{ll}
A_{3}=\frac{6 m_{3}}{L} ; & C_{3}=\frac{3 M_{3}}{L} ; \quad b_{3}=-b_{2}=\frac{12 m_{3}}{L^{2}} \\
m_{2}=5 m_{3} ; & M_{2}=2 M_{3}
\end{array}
$$

obtêm-se os resultados:

$$
\begin{aligned}
& -c_{S}^{2} \frac{b_{3} \cos \left(\alpha_{N} L\right)-b_{2}}{m_{3} \cos \left(\alpha_{N} L\right)-m_{2}}=c_{S}^{2}\left(\alpha_{N}^{2}+O\left[L^{4}\right]\right) \\
& c_{S}^{2} \frac{A_{3} \operatorname{sen}\left(\alpha_{N} L\right)}{m_{3} \cos \left(\alpha_{N} L\right)-m_{2}}=c_{S}^{2}\left(i \alpha_{N}-\frac{i \alpha_{N}^{3}}{12} L^{2}+O\left[L^{4}\right]\right) \\
& -r^{2} \frac{c_{3} \operatorname{sen}\left(\alpha_{N} L\right)}{M_{3} \cos \left(\alpha_{N} L\right)-M_{2}}=r^{2}\left(-i \alpha_{N}+O\left[L^{4}\right]\right)
\end{aligned}
$$

Substituindo-se de forma direta (6.20) em (6.15), chega-se finalmente a: 


$$
\begin{aligned}
& \ddot{y}_{n, j}+c_{S}^{2} \alpha_{N}^{2} y_{n, j}+i c_{S}^{2} \alpha_{N} \theta_{n, j}-c_{S}^{2} \frac{i \alpha_{N}^{3}}{12} L^{2} \theta_{n, j}+O\left[L^{4}\right]=0 \\
& \ddot{y}_{n, j}-r^{2} i \alpha_{N} \ddot{\theta}_{n, j}+O\left[L^{4}\right]=0
\end{aligned}
$$

Percebe-se, pois, que, devido ao fato da impossibilidade de se aproximar numericamente a derivada terceira com apenas três pontos, a expressão (6.11b) contém apenas a contribuição da aceleração angular da seção transversal. A combinação dos termos discretos, como sabido, é dada por:

$$
\begin{aligned}
& \stackrel{m}{\nabla} \ddot{y}_{n, j}-c_{s}^{2} \stackrel{b}{\nabla} y_{n, j}+c_{s}^{2} \stackrel{A}{\nabla} \theta_{n, j}=0 \\
& \stackrel{M}{\nabla} \ddot{y}_{n, j}-r^{2} \stackrel{c}{\nabla} \ddot{\theta}_{n, j}=0
\end{aligned}
$$

que, quando aplicado (6.10), retorna:

$$
\begin{aligned}
& \stackrel{m}{\nabla} \ddot{y}_{n, j}+\stackrel{m}{\nabla} c_{S}^{2} \alpha_{N}^{2} y_{n, j}+\stackrel{m}{\nabla} c_{S}^{2} i \alpha_{N} \theta_{n, j}-\stackrel{m}{\nabla} c_{S}^{2} \frac{i \alpha_{N}^{3}}{12} L^{2} \theta_{n, j}+O\left[L^{4}\right]=0 \\
& \stackrel{M}{\nabla} \ddot{y}_{n, j}-\stackrel{M}{\nabla} r^{2} i \alpha_{N} \ddot{\theta}_{n, j}+O\left[L^{4}\right]=0
\end{aligned}
$$

A idéia agora é combinar estas duas expressões de tal sorte que a parcela que contém $i \alpha_{N}{ }^{3}$ em (6.23a) seja o complemento de (6.23b) para a equação teórica (6.13b). Assim, aplicando um fator $\mu$ para (6.23a) e (1- $\mu$ ) para (6.23b) e somando-as em seguida, chega-se a uma única expressão na forma:

$$
\mu \stackrel{m}{\nabla}\left(\ddot{y}_{n, j}+c_{S}^{2} \alpha_{N}^{2} y_{n, j}+c_{S}^{2} i \alpha_{N} \theta_{n, j}-c_{S}^{2} \frac{i \alpha_{N}^{3}}{12} L^{2} \theta_{n, j}\right)+\stackrel{M}{\nabla}(1-\mu)\left(\ddot{y}_{n, j}-r^{2} i \alpha_{N} \ddot{\theta}_{n, j}\right)+O\left[L^{4}\right]=0
$$

Deseja-se, portanto, encontrar o valor de $\mu$ que conduza à igualdade:

$$
-\mu \stackrel{m}{\nabla} c_{S}^{2} \frac{i \alpha_{N}^{3}}{12} L^{2} \theta_{n, j}=(1-\mu) \stackrel{M}{\nabla} c_{R}^{2} r^{2}\left(i \alpha_{N}\right)^{3} \theta_{n, j}
$$
quando:

Isolando-se agora o termo $\mu$, conclui-se que esta expressão é obedecida 


$$
\mu=\frac{12 \sigma \gamma^{2} \delta^{2}}{1+12 \sigma \gamma^{2} \delta^{2}}
$$

onde:

$$
\sigma=\frac{\stackrel{M}{m}}{\nabla}=\frac{M_{3}}{m_{3}}\left(\frac{2+\cos \left(\alpha_{N} L\right)}{5+\cos \left(\alpha_{N} L\right)}\right)=\frac{M_{3}}{2 m_{3}}-\frac{M_{3} \alpha_{N}^{2} L^{2}}{24 m_{3}}+O\left[L^{4}\right]
$$

Por se tratar de um mesmo caso no ponto de vista físico, a inércia linear de ambos os casos deve ser igual, isto é:

$$
m_{1}+2 m_{2}+m_{3}=M_{1}+2 M_{2}+M_{3}
$$

Implicando-se que:

$$
12 m_{3}=6 M_{3} \Rightarrow \frac{M_{3}}{m_{3}}=2
$$

Finalmente, o valor de $\mu$ pode ser resumido como:

$$
\mu=\frac{12 \gamma^{2} \delta^{2}}{1+12 \gamma^{2} \delta^{2}}+\mathrm{O}\left[\mathrm{L}^{2}\right]
$$

Valendo-se ressaltar que a combinação das equações combinatórias são válidas mesmo quando o valor de $\mu$ contém uma parcela de ordem de erro (ou seja, independe do valor numérico de $\mu$ ). Aponta-se também que o valor obtido para $\mu$ é exatamente a razão $\Phi /(\Phi+1)$, presente na formulação clássica de elemento simples de viga segundo Timoshenko.

Desenvolvendo-se a igualdade (6.25) com conseqüente substituição de (6.30):

$$
-\frac{12 \gamma^{2} \delta^{2}}{1+12 \gamma^{2} \delta^{2}} \stackrel{m}{\nabla} c_{S}^{2} \frac{i \alpha_{N}^{3}}{12} L^{2} \theta_{n, j}+O\left[L^{4}\right]=(1-\mu) \stackrel{M}{\nabla} c_{R}^{2} r^{2}\left(i \alpha_{N}\right)^{3} \theta_{n, j}
$$

podendo-se concluir que o valor encontrado para $\mu$ retorna uma aproximação com erro de quarta potência em L. Deduz-se que, embora $\mu$ contemple um erro de segunda ordem, o erro geral é $O\left[L^{4}\right]$, pois $\mu$ multiplica um termo proporcional a $L^{2}$. 
Concluindo-se, a substituição direta de (6.31) em (6.24) denota uma aproximação numérica que obedece, a menos de uma ordem de erro $O\left[L^{4}\right]$, à combinação entre as duas formas das equações de equilíbrio de forças transversais, conforme exibido na expressão seguinte:

$$
\mu \stackrel{m}{\nabla}\left(\ddot{y}_{n, j}+c_{S}^{2} \alpha_{N}^{2} y_{n, j}+c_{S}^{2} i \alpha_{N} \theta_{n, j}\right)+\stackrel{M}{\nabla}(1-\mu)\left(\ddot{y}_{n, j}-r^{2} i \alpha_{N} \ddot{\theta}_{n, j}+c_{R}^{2} r^{2}\left(i \alpha_{N}\right)^{3} \theta_{n, j}\right)+O\left[L^{4}\right]=0(6.32)
$$




\section{CAPÍtulo 7}

\section{Conclusões}

Os métodos de integração propostos demonstram, de fato, eficiência elevada na precisão e na estabilidade da resposta de um sistema linear dinâmico discretizado. As figuras 3.1b (elemento de barra), 5.1 e 5.10 (elemento de viga) esclarecem que uma precisa integração no tempo e no espaço $\left(\beta=1 / 12\right.$ e $\left.\beta_{S}=1 / 12\right)$ retorna valores muito mais próximos dos teóricos. Isso possibilita a geração de malhas menos refinadas sem o comprometimento da precisão do modelo. Observando ainda as tabelas 3.1 e 3.2 , nota-se que a resposta do modelo de ordem elevada tem desempenho muito superior ao modelo consistente clássico.

Tão importante quanto a precisão numérica, outro problema enfrentado em análises estruturais através do método dos elementos finitos e a instabilidade do 
sistema perante fenômenos espúrios [BAZANT (1978), JIANG (1991), LAIER (2002)]. A solução encontrada, inspirada no integrador incondicionalmente estável de Newmark [Newmark (1959)], demonstra, de acordo com as figuras 3.3d, 3.4, 3.5, 5.8 e 5.9, isenção do efeito de onda evanescente e da reflexão espúria.

Portanto, trata-se de duas famílias distintas de elementos finitos $\left(\beta_{S}=1 / 12\right.$ e $\left.\beta_{S}=1 / 4\right)$. Naturalmente, o calculista estrutural é quem deve optar pela precisão ou estabilidade, tomando esta decisão em função de cada caso.

Além das vantagens apontadas, as matrizes de massa aqui propostas desacoplam a inércia de translação e de rotação, garantindo não só menor custo computacional como também a aplicação de massa concentrada em métodos explícitos.

Complementarmente, é praticamente intuitiva a conclusão de que a adição de nós em elementos finitos implicariam, simplesmente, na adição de termos trigonométricos na equação discreta de equilíbrio de ações. Por exemplo, no caso do elemento de barra, a adoção de um terceiro nó no centro geométrico da barra alteraria a equação de autovalores (3.10) para:

$$
\ddot{u}_{j}^{*}+\left[\frac{k_{1} \cos \left(\alpha_{N} L\right)+k_{2} \cos \left(\frac{\alpha_{N} L}{2}\right)+k_{3}}{m_{1} \cos \left(\alpha_{N} L\right)+m_{2} \cos \left(\frac{\alpha_{N} L}{2}\right)+m_{3}}\right] u_{j}^{*}=0
$$

Embora não seja usual o emprego de elementos com um número elevado de nós, pode-se então generalizar para o caso de um elemento com $\mathrm{N}$ nós através da redação:

$$
\ddot{u}_{j}^{*}+\frac{\sum_{s=1}^{N} k_{N-s+1} \cos \left(\alpha_{N} \frac{s-1}{N-1} L\right)}{\sum_{j=1}^{N} m_{N-s+1} \cos \left(\alpha_{N} \frac{s-1}{N-1} L\right)} u_{j}^{*}=0
$$

Similarmente, a formulação genérica para elementos de viga com $\mathrm{N}$ nós eqüidistantes é: 


$$
\begin{gathered}
\ddot{y}_{j}^{*}+\frac{\sum_{s=1}^{N} k_{N-s+1} \cos \left(\alpha_{N} \frac{s-1}{N-1} L\right)}{\sum_{j=1}^{N} m_{N-s+1} \cos \left(\alpha_{N} \frac{s-1}{N-1} L\right)} y_{j}^{*}+\frac{\sum_{s=1}^{N-1} K_{N-s} \operatorname{sen}\left(\alpha_{N} \frac{s}{N-1} L\right)}{\sum_{j=1}^{N} m_{N-s+1} \cos \left(\alpha_{N} \frac{s-1}{N-1} L\right)} \theta_{j}^{*}=0 \\
\ddot{\theta}_{j}^{*}+\frac{\sum_{s=1}^{N-1} \bar{k}_{N-s} \operatorname{sen}\left(\alpha_{N} \frac{s}{N-1} L\right)}{\sum_{j=1}^{N} \bar{M}_{N-s+1} \cos \left(\alpha_{N} \frac{s-1}{N-1} L\right)} y_{j}^{*}+\frac{\sum_{s=1}^{N} \bar{K}_{N-s+1} \cos \left(\alpha_{N} \frac{s-1}{N-1} L\right)}{\sum_{j=1}^{N} \bar{M}_{N-s+1} \cos \left(\alpha_{N} \frac{s-1}{N-1} L\right)} \theta_{j}^{*}=0
\end{gathered}
$$

Convenientemente, ao se trabalhar com parâmetros adimensionalizados com a conveniente escolha dos parâmetros $p$ e $\xi$ (que exprimem o grau de discretização no espaço e no tempo respectivamente), é permitido interpretar os resultados genericamente.

É interessante apontar que a formulação proposta tem aplicabilidade em outros tipos de elementos finitos em regime linear, tal qual o de transferência térmica, acústico e fluido. Outras propriedades do elemento finito estrutural também podem ser abrangidas, como instabilidade (flambagem), fluência, relaxação e histerese. Ou seja, qualquer fenômeno físico que seja regido por uma equação diferencial pode ser transposto ao sistema discretizado, resultando uma equação de diferenças finitas, possibilitando a elevação da ordem de erro tanto no domínio do tempo quanto no domínio do espaço. Devido a este fato, talvez a validação da formulação proposta para elementos finitos dinâmicos possibilite novos recursos para elaboração de algoritmos numéricos de integração.

Enfim, atualmente encontram-se inúmeros estudos que buscam melhor desempenho de elementos finitos através da modificação das funções de forma. Tal procedimento geralmente se encontra atrelado ao método de mínimo potencial total. O diferencial deste trabalho é modelar um método alternativo a este, cujo alvo é explicitar a ordem de erro relativo ao número de onda e à freqüência de oscilação. 


\section{REFERÊNCIAS}

AHMADIAN, H.; FRISWELL, M.; MOTTERSHEAD, J. Minimization of the error in mass and stiffness formulations by an inverse method. International Journal for Numerical Methods in Engineering, vol. 41, 1998.

BAZANT, Z. Spurious reflection of elastic waves in non-uniform finite element grids. Computer Methods in Applied Mechanics and Engineering, vol. 16, 1978.

BILLGER, D.; FOLKOW, P. The imbedding equations for the Timoshenko beam. Journal of Sound and Vibration, vol. 209(4), 1998.

COWPER, G. The shear coefficient in Timoshenko's beam theory. Journal of Applied Mechanics, 1966.

FRANKLIN, Y. Vibrations of Timoshenko beams and frameworks. Journal of the Structural Division, 1970.

JIANG, L.; ROGERS, R. Spurious wave reflections at an interface of different physical properties in finite-element wave solutions. Communications in Applied Numerical Methods, vol. 7, 1991.

LAIER, J. Estudo dos movimentos espúrios produzidos por integradores de ordem elevada. In: XXIX JORNADAS SULAMERICANAS DE ENGENHARIA ESTRUTURAL, 2000. Anais.

. Dispersive and spurious reflections of Timoshenko's flexural waves in numerical simulations. Advances in Engineering Software, vol. 33, 2002.

LIU, J.; SHARAN, S.; YAO, L. Wave motion and its dispersive properties in a finite element model with distortional elements. Computers \& Structures, vol. 52, n 2, 1994.

LUNDÉN, R.; AKESSON, B. Damped second-order Rayleigh-Timoshenko beam vibration in space - an exact complex dynamic member stiffness matrix. International Journal fro Numerical Methods in Engineering, vol. 19, 1983.

MIKLOWITZ, J. Recent developments in elastic wave propagation, Applied Mechanics Reviews, vol. 13, nº 12, 1960.

MULDER, W. Spurious modes in finite-element discretizations of the wave equation may not be all that bad. Applied Numerical Mathematics, vol. 30, 1999. 
MULLEN, R.; BELYTSCHKO, T. Dispersion analysis of finite element semidiscretizations of the two-dimensional wave equation. International Journal for Numerical Methods in Engineering, vol. 18, 1982.

NEWMARK, N. A method of computation for structural dynamics. ASCE Journal of the Engineering Mechanics, Division 85, 1959.

NORONHA, C.; LAIER, J. Spurious wave reflections in Timoshenko beam elements. In: XXV CILAMCE, 2004, Recife-PE. Anais.

Analytical expressions for numerical dispersion and spurious wave reflections in Timoshenko beam elements. In: XXVI CILAMCE, 2005, Guarapari-ES. Anais.

. Integradores de elementos finitos de barra de elevada ordem, reduzidas reflexões espúrias e incondicionalmente estáveis. In: XXXII Jornadas Sulamericanas de Engenharia Estrutural, 2006, Campinas-SP. Anais.

Estudo das amplitudes e velocidades numéricas das ondas refletidas e transmitidas em malhas não-homogêneas em elementos finitos de barra. IV DINCON, 2005, Bauru-SP. Anais.

SCHREYER, $H$. Consistent diagonal mass matrices and finite element equations for one-dimensional problems. International Journal for Numerical Methods in Engineering, vol. 12, 1978.

THOMAS, D.; WILSON, J.; WILSON, R. Thimoshenko beam finite elements. Journal of Sound and Vibration, vol. 31, 1973.

WANG, Y.; VIRIYAWAN, M.; VALLIAPPAN, S. Assessment of the accuracy of the Newmark method in transient analysis of wave propagation problems, Earthquake Engineering and Structural Dynamics, vol. 21, 1992. 